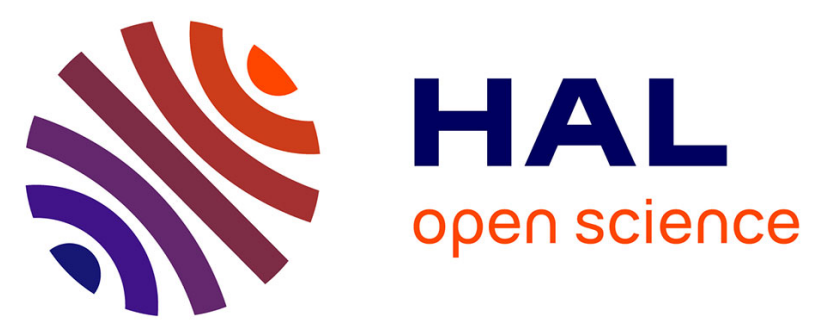

\title{
Image Simulation and Assessment of the Colour and Spatial Capabilities of the Colour and Stereo Surface Imaging System (CaSSIS) on the ExoMars Trace Gas Orbiter
}

Livio Tornabene, Frank Seelos, Antoine Pommerol, Nicholas Thomas, C. Caudill, Patricio Becerra, C. Bridges, Shane Byrne, Marco Cardinale, Matthew Chojnacki, et al.

\section{- To cite this version:}

Livio Tornabene, Frank Seelos, Antoine Pommerol, Nicholas Thomas, C. Caudill, et al.. Image Simulation and Assessment of the Colour and Spatial Capabilities of the Colour and Stereo Surface Imaging System (CaSSIS) on the ExoMars Trace Gas Orbiter. Space Science Reviews, 2018, 214 (1), 10.1007/s11214-017-0436-7 . hal-02270615

\section{HAL Id: hal-02270615 \\ https://hal.science/hal-02270615}

Submitted on 8 Jan 2021

HAL is a multi-disciplinary open access archive for the deposit and dissemination of scientific research documents, whether they are published or not. The documents may come from teaching and research institutions in France or abroad, or from public or private research centers.
L'archive ouverte pluridisciplinaire HAL, est destinée au dépôt et à la diffusion de documents scientifiques de niveau recherche, publiés ou non, émanant des établissements d'enseignement et de recherche français ou étrangers, des laboratoires publics ou privés. 
1 Image simulation and assessment of 2 the colour and spatial capabilities of 3 the Colour and Stereo Surface 4 Imaging System (CaSSIS) on the 5 ExoMars Trace Gas Orbiter

6 Livio L. Tornabene ${ }^{1}$, Frank. P. Seelos ${ }^{2}$, Antoine Pommerol ${ }^{3}$, Nicholas Thomas ${ }^{3}$, C.M. 7 Caudill $^{1}$, Patricio Becerra ${ }^{3}$, John C. Bridges ${ }^{4}$, Shane Byrne ${ }^{5}$, Marco Cardinale ${ }^{6}$, 8 Matthew Chojnacki $^{5}$, Susan J. Conway ${ }^{7}$, Gabriele Cremonese $^{8}$, Colin M. Dundas ${ }^{9}$, 9 M. R. El-Maarry ${ }^{10}$, Jennifer Fernando ${ }^{5}$, Candice J. Hansen ${ }^{11}$, Kayle Hansen ${ }^{1}$, Tanya 10 N. Harrison ${ }^{12}$, Rachel Henson ${ }^{4}$, Lucia Marinangeli ${ }^{6}$, Alfred S. McEwen ${ }^{5}$, Maurizio 11 Pajola $^{13}$, Sarah S. Sutton ${ }^{5}$ and James J. Wray ${ }^{14}$

$12{ }^{1}$ Centre for Planetary Science and Exploration/Department of Earth Sciences, University of 13 Western Ontario, London, Ontario N6A 5B7, Canada (E-mail: Itornabe@uwo.ca)

14 2Johns Hopkins University Applied Physics Laboratory, Laurel, Maryland, USA

15 3Physikalisches Institut, Sidlerstr. 5, University of Bern, CH-3012 Bern, SWITZERLAND

$16{ }^{4}$ Space Research Centre, Leicester Institute for Space and Earth Observation, University of Leicester, 17 UK LE1 7RH

18 5unar and Planetary Laboratory, University of Arizona, Tucson, Arizona 85721-0092, USA

$19{ }^{6}$ DiSPUTER, Università G.d'Annunzio, Chieti, Italy

$20{ }^{7}$ CNRS, Laboratoire de Planétologie et Géodynamique, CNRS/INSU UMR 6112, Université de Nantes,

212 chemin de la Houssinière, BP 92205, 44322 Nantes Cedex 3, France.

22 8INAF-Osservatorio Astronomicodi Padova, Vicolo Osservatorio 5, 35122 Padova, Italy

$23{ }^{9}$ U.S. Geological Survey, Astrogeology Science Center, 2255 N. Gemini Dr., Flagstaff, AZ 86001, USA.

$24{ }^{10}$ Laboratory of Atmospheric and Space physics (LASP), University of Colorado, 3665 Discovery Dr., 25 Boulder, CO 80303, USA.

$26{ }^{11}$ Planetary Science Institute, 1700 E. Fort Lowell, Suite 106, Tucson, AZ 85719, USA

$27{ }^{12}$ NewSpace Initiative, Arizona State University, P.O. Box 876004, Tempe, AZ 85287

$28{ }^{13}$ NASA Ames Research Center, Moffett Field, CA 94035, USA.

$29{ }^{14}$ School of Earth and Atmospheric Sciences, Georgia Institute of Technology, Atlanta, GA 30332, 30 USA.

Keywords: Mars; Mars, geology; Mars, surface processes; Mars, climate; Mars, change detection; Mars, landing sites; multispectral imaging; image processing; band ratios; pan-sharpening 


\section{ABSTRACT}

36 This study aims to assess the spatial and visible/near-infrared (VNIR) colour/spectral capabilities

37 of the 4-band Colour and Stereo Surface Imaging System (CaSSIS) aboard the ExoMars 2016 Trace

38 Grace Orbiter (TGO). The instrument response functions for the CaSSIS imager was used to

39 resample spectral libraries, modelled spectra and to construct spectrally (i.e., in I/F space) and

40 spatially consistent simulated CaSSIS image cubes of various key sites of interest and for ongoing

41 scientific investigations on Mars. Coordinated datasets from Mars Reconnaissance Orbiter (MRO)

42 are ideal, and specifically used for simulating CaSSIS. The Compact Reconnaissance Imaging

43 Spectrometer for Mars (CRISM) provides colour information, while the Context Imager (CTX), and

44 in a few cases the High-Resolution Imaging Science Experiment (HiRISE), provides the

45 complementary spatial information at the resampled CaSSIS unbinned/unsummed pixel

46 resolution (4.6 $\mathrm{m} / \mathrm{pixel}$ from a $400-\mathrm{km}$ altitude). The methodology used herein employs a Gram-

47 Schmidt spectral sharpening algorithm to combine the $18-36 \mathrm{~m} /$ pixel CRISM-derived CaSSIS

48 colours with $\mathrm{I} / \mathrm{F}$ images primarily derived from oversampled CTX images. One hundred and

49 eighty-one simulated CaSSIS 4-colour image cubes (at 18-36 m/pixel) were generated (including

50 one of Phobos) based on CRISM data. From these, thirty-three "fully"-simulated image cubes of

51 thirty unique locations on Mars (i.e., with 4 colour bands at $4.6 \mathrm{~m} / \mathrm{pixel}$ ) were made. All

52 simulated image cubes were used to test both the colour capabilities of CaSSIS by producing

53 standard colour RGB images, colour band ratio composites (CBRCs) and spectral parameters.

54 Simulated CaSSIS CBRCs demonstrated that CaSSIS will be able to readily isolate signatures

55 related to ferrous $\left(\mathrm{Fe}^{2+}\right)$ iron- and ferric $\left(\mathrm{Fe}^{3+}\right)$ iron-bearing deposits on the surface of Mars, ices

56 and atmospheric phenomena. Despite the lower spatial resolution of CaSSIS when compared to

57 HiRISE, the results of this work demonstrate that CaSSIS will not only compliment HiRISE-scale

58 studies of various geological and seasonal phenomena, it will also enhance them by providing

59 additional colour and geologic context through its wider and longer full-colour coverage ( $\sim 9.4 \mathrm{x}$

$6050 \mathrm{~km}$ ), and its increased sensitivity to iron-bearing materials from its two IR bands (RED and

61 NIR). In a few examples, subtle surface changes that were not easily detected by HiRISE were

62 identified in the simulated CaSSIS images. This study also demonstrates the utility of the Gram-

63 Schmidt spectral pan-sharpening technique to extend VNIR colour/spectral capabilities from a

64 lower spatial resolution colour/spectral dataset to a single-band or panchromatic image grayscale

65 image with higher resolution. These higher resolution colour products (simulated CaSSIS or

66 otherwise) are useful as means to extend both geologic context and mapping of datasets with

67 coarser spatial resolutions. The results of this study indicate that the TGO mission objectives, as

68 well as the instrument-specific mission objectives, will be achievable with CaSSIS. 
The payload of the ExoMars 2016 Trace Grace Orbiter (TGO) (Vago et al

71 2015) is designed to detect and characterise the concentration and spatial

72 distribution of trace gases in the Martian atmosphere over various times of day,

73 incidence and spanning all the Martian seasons. Methane $\left(\mathrm{CH}_{4}\right)$, a metastable and

74 short-lived trace gas linked to life and/or active geological processes, was

75 putatively detected on Mars from Earth-based, orbital and surface measurements

76 (e.g., Formisano et al 2004; Mumma et al 2009; Fonti and Marzo 2010; Villanueva

77 et al 2013; Webster et al 2015) and was the catalyst for this mission (Zurek et al

78 2011). The Colour and Stereo Surface Imaging System (CaSSIS) is a moderately

79 high-resolution 4-colour visible/near-infrared (VNIR) stereo camera included as

80 part of the payload of the TGO (Thomas et al this issue). As a VNIR imager pointed

81 at the surface of Mars, CaSSIS is specifically tasked with: 1) characterising possible

82 [surface/subsurface] sources for methane and other trace gases; 2) investigating

83 dynamic surface processes that may contribute to atmospheric gases; and 3)

84 certifying and characterising safety and hazards (e.g., rocks, slopes, etc.)

85 associated with candidate landing sites for ExoMars 2020 and other future surface

86 missions. Furthermore, and similarly to the High-Resolution Imaging Science

87 Experiment (HiRISE) (McEwen et al 2007, 2010) on the Mars Reconnaissance

88 Orbiter (MRO) [Zurek and Smrekar 2007], investigations by the members of the

89 CaSSIS team emphasize a variety of Mars science objectives organized under

90 specific science theme groups (e.g., seasonal processes, impact cratering, etc.).

91 This ensures that CaSSIS will cover diverse areas of study that include and go

92 beyond the focused science objectives of CaSSIS and TGO. The instrument design

93 and anticipated CaSSIS observations are expected to provide high-resolution and

94 full-colour stereo products that will highly complement past, present, and future

95 datasets.

96 This study seeks to assess and simulate the colour and spatial capabilities

97 of CaSSIS and thereby assess how CaSSIS, and its various expected high-level

98 derived data products, will be used to meet both orbiter and instrument

99 objectives, and evaluate to what extent CaSSIS falls short of, complements, or in

100 some cases even exceeds the capabilities of previous imagers (e.g., HiRISE). 
101 Despite the significantly higher spatial resolution of HiRISE, CaSSIS offers three

102 notable advantages over HiRISE including: 4-band colour, a wider image swath,

103 and colour capability across the entire image. CaSSIS can also acquire along-track

104 stereo images on a single orbital pass, and, due to the orbital inclination of the

105 TGO, it can obtain images over a range in local times and incidence; however, we

106 note here that these particular characteristics are not a major focus of this

107 simulation study (for more details, see Thomas et al this issue).

108 In this study, we begin by making use of the CaSSIS instrument spectral 109 response functions (i.e., bandpasses) to evaluate CaSSIS colour sensitivity to 110 various minerals and Fe-bearing surface materials known to exist on Mars. In 111 addition to this, we have developed methods for simulating CaSSIS observations

112 and data products using complementary and coordinated MRO datasets covering 113 various sites of interest on Mars. These simulated CaSSIS products cover spectral 114 mineral/phase type-localities, key morphologic features related to dynamic 115 surface processes, and high-priority landing sites. These simulated CaSSIS 4-band 116 colour image cubes are then used to assess to what extent CaSSIS will continue 117 and expand upon ongoing investigations of various surface processes monitored 118 by HiRISE and previous imagers, such as the Mars Orbiter Camera (MOC), the High119 Resolution Stereo Camera (HRSC), and the Context Imager (CTX). The results of our 120 study herein are anticipated to also help the CaSSIS science and operations teams 121 to determine the best higher-level data products to produce, and best practices

122 for planning targeted colour and stereo observations, and aid colour calibration 123 efforts. As such, this work will enable the team to optimize both operations and 124 maximise the science return for the mission. Lastly, the methods used here to 125 create fully-simulated CaSSIS image cubes provides the means to extend the 126 monitoring of long-term surface changes on Mars with CaSSIS to the pre-TGO era 127 (i.e., back to the onset of the MRO mission in late 2006) by providing a spectrally 128 and spatially consistent MRO-derived CaSSIS-compatible product that may be 129 used to for comparison to actual CaSSIS data to be acquired in 2018 and beyond.

\section{$130 \quad 1.1$ CaSSIS instrument and colour band selection}

131 CaSSIS is a pushframe imager that will provide colour images of the Martian 132 surface at $\sim 4.6 \mathrm{~m} / \mathrm{pixel}$, which will be up to $\sim 9.4 \mathrm{~km}$ wide and possibly up to $\sim 40-$ 
13350 kilometres long from a final circularized orbital altitude of $\sim 400 \mathrm{~km}$. CaSSIS has

134 a fixed pointing of $\sim 10^{\circ}$ off nadir and a rotation mechanism that is used to acquire 135 stereo images in a single orbital pass providing a $22.14^{\circ}$ parallax angle. Unlike all 136 other recent Mars orbiters with high-resolution cameras, the final configuration 137 of the TGO orbit will be at an inclination of $74^{\circ}$ to the equator; although this will 138 limit the ability of CaSSIS to image targets above $74^{\circ} \mathrm{N}$ or $\mathrm{S}$, it provides CaSSIS the 139 means to image at multiple times of day across all seasons and over a larger range 140 of incidence angles.

141 The pushframe imaging configuration utilized by CaSSIS offers some 142 advantages with respect towards achieving a good radiometric calibration solution 143 for colour CaSSIS images of Mars; this is further supported by recent results from 144 the ground calibration effort thus far, which is showing very small errors against 145 standard stars (see Roloff et al this issue). For more detailed information on design 146 and ground calibration of the CaSSIS imaging system, please see Thomas et al and 147 Roloff et al, respectively, this issue.

148 The 4 colour filters of CaSSIS correspond to blue-green (BLU), broadband 149 orange-red (PAN), and two near-infrared colours (RED and NIR), collectively 150 utilizing a detector sensitivity that spans $\sim 400$ to $1100 \mathrm{~nm}$ (Table 1). This 151 wavelength sensitivity range is notably similar to HiRISE (Delamere et al 2010; 152 McEwen et al 2010). The first two CaSSIS bands, blue-green (BLU) and a broadband 153 red (PAN), are very similar to BG and RED filters of HiRISE, respectively (c.f., 154 Delamere et al 2010). With respect to the NIR, CaSSIS provides two bands (RED 155 and NIR) that effectively split the single HiRISE IR band. These two CaSSIS NIR filters 156 are expected to provide extra sensitivity to the detection of Fe-bearing surface 157 materials.

158 Thee four CaSSIS bands will provide a Signal-to-Noise Ratio (SNR) that is $159 \sim 50 \%$ higher than the 3 bands of HiRISE, or $7 \%$ higher SNR ( $>100: 1$ top of 160 atmosphere) based on Mars reference surfaces under similar observational 161 conditions (Thomas et al this issue; Roloff et al this issue). Based on these 162 characteristics, CaSSIS will not only complement HiRISE colour products, but will 163 also provide similar sensitivities to characteristic spectral features in this range 164 that are useful for distinguishing Fe-bearing materials on Mars, as well as ices and 
165 various atmospheric phenomena (e.g., haze, fog, clouds, dust devils, avalanche 166 clouds, etc.).

167 The reflectivity or $\mathrm{I} / \mathrm{F}$ intensity of $\mathrm{CO}_{2}$ and $\mathrm{H}_{2} \mathrm{O}$ ice- and frost-bearing 168 surfaces are higher in blue wavelengths compared to other Mars surface materials 169 (e.g., James et al 1994; Bell et al 1997). The reflectivity of $\mathrm{H}_{2} \mathrm{O}$ is relatively lower 170 than $\mathrm{CO}_{2}$ ice in red and NIR wavelengths, due to a spectral slope towards the NIR 171 and the presence of a relatively narrow and shallow absorption feature centred 172 around $1030 \mathrm{~nm}$ (Figure 1) (e.g., Williams and Ferrigno 1988; Becerra et al 2015).

173 Furthermore, surfaces containing fine-grained ice or frost, especially if the

174 ice/frost is transparent to translucent, will likely have high reflectance values over 175 the entire 400-1100 nm sensitivity range of CaSSIS (e.g., Appéré et al 2011), when 176 compared to ice-free surfaces. Like ice/frost, various atmospheric phenomena 177 (e.g., fog, haze, clouds, active dust devils, avalanche clouds, etc.) also have 178 relatively higher reflectance values over the visible spectrum, and especially over

179 the bluer wavelengths and when compared to the Martian surface that lacks ice 180 or frost (e.g., James et al 1994; Bell et al 1997).

\section{1 [FIGURE 1]}

182 The VNIR colour of metal-bearing minerals is a consequence of metallic 183 cations in the periodic table's first row of transition elements (i.e., Ti to $184 \mathrm{Cu}$ ). However, the colours observed in the most common rock forming minerals 185 are often attributed to variations in the oxidation state of iron $\left(\mathrm{Fe}^{2+}, \mathrm{Fe}^{3+}\right)$ and the 186 crystalline structure (or lack thereof) of the material (Figure 1). Specifically, within 187 the wavelength sensitivity range of CaSSIS, there are electronic transitions and 188 crystal field effects due to the presence of ferrous $\left(\mathrm{Fe}^{2+}\right)$ iron-bearing minerals 189 responsible for strong and diagnostic absorptions at $700-1100 \mathrm{~nm}$ (e.g., mafic 190 minerals such as olivine and pyroxene). The CaSSIS sensitivity range also includes 191 diagnostic broad absorptions extending from the UV to the visible, short of 550 $192 \mathrm{~nm}$, which arise from intervalence charge-transfer transitions of ferric iron $\mathrm{Fe}^{3+}$ 193 and $\mathrm{O}^{2-}$ present in altered ferric $\left(\mathrm{Fe}^{3+}\right)$ iron-bearing minerals (e.g., hematite, 194 nontronite, etc.) (e.g., Adams 1974; Hunt 1977; Hunt and Ashley 1979; Burns 195 1993). Although colour can be characteristic of specific minerals, it is not 196 necessarily diagnostic. However, colour CaSSIS images (especially band ratios) are 
197 expected to be quite helpful towards discriminating ferrous vs. ferric materials,

198 ice- or frost-bearing surfaces, and extending spectral mapping from orbital

199 instruments with coarser spatial scales, such as the Compact Reconnaissance

200 Imaging Spectrometer for Mars (CRISM) and the Observatoire pour la Mineralogie,

201 l'Eau, la Glace et l'Activite (OMEGA).

202 This knowledge of the general spectral behaviour over the CaSSIS

203 wavelength sensitivity range that are due to the presence of various surface

204 materials/atmospheric phenomena (summarized above) is utilized, in conjunction

205 with the CaSSIS band positions, to formulate spectral ratios and parameters that

206 will be used to isolate pixels with high-concentrations or good exposures of these

207 materials on Mars (see Section 3).

\section{Methods}

$209 \quad 2.1$ CaSSIS colour-sensitivity to known minerals on Mars

210 Here we use the CaSSIS instrument response functions to resample, and

211 then subsequently analyse, lab-measured spectra for selected minerals that are

212 known to exist on Mars. A model for the instrument response and spectral

213 sensitivity of CaSSIS (see Figure 2), from approximately 400 to $1100 \mathrm{~nm}$, was built

214 from manufacturer-provided spectral curves for each of the relevant instrument

215 elements, the quantum efficiency of the detector, the transmission of the

216 bandpass filters and the reflectance of the four-mirror telescope optics (Thomas

217 et al this issue). All response curves were measured with a spectral sampling of 1

$218 \mathrm{~nm}$. These response curves were then verified in the laboratory during the pre-

219 flight calibration of the instrument. See Roloff et al (this issue) for a discussion of

220 the observed agreements and discrepancies during pre-flight calibration.

221 The minerals and phases selected for this study are based on published

222 summaries of spectral studies of the Martian surface, in situ detections and the

223 known composition of Martian meteorites (see Ehlmann et al 2011; Carter et al

224 2013; Ehlmann and Edwards 2014; and Viviano-Beck et al 2014 and references

225 therein). The equivalent lab-measured spectra for Martian minerals and phases

226 are derived from the USGS and CRISM mineral spectral libraries (e.g., Clark et al 
228 2017).

\section{9 [FIGURE 2]}

\section{$230 \quad 2.2$ Creating simulated CaSSIS products}

231 The second part of this study entails the creation and assessment of 232 simulated CaSSIS image cubes covering various surface materials, landforms and 233 other sites of interest on Mars based on MRO datasets. For this purpose, we 234 created two types of simulated cubes: "partial" - ones that simulate the CaSSIS 235 spectral/colour bands only, and "fully" - ones that simulate CaSSIS both spectrally 236 (4 bands) and spatially (w.r.t. pixel-scale). We note here that our simulated CaSSIS 237 cubes do not account for differences in modular transfer functions, geometric 238 distortion, system noise, SNR, and atmospheric effects that will inherently vary 239 with respect to the MRO data sources used to create a specific simulated CaSSIS 240 cube and the actual CaSSIS images that will be acquired in the future. Nonetheless, 241 these simulated products will enable us to begin to assess the general 242 spectra/colour and spatial capabilities of CaSSIS.

243 Simulated CaSSIS spectral/colour image cubes are initially created by 244 convolving VNIR spectral information obtained by the CRISM instrument (Murchie 245 et al 2007) with the CaSSIS instrument response functions. However, these initial 246 colour products maintain the spatial scale of the input CRISM cubes (i.e., $18-36$ $247 \mathrm{~m} / \mathrm{pixel}$ ) and constitutes our "partial" simulated product. These colour-only 248 simulated products come as two types of image cubes, a "CRISM-CaSSIS 249 Compatible" $\left(\mathrm{C}^{3}\right)$, and a "Corrected CRISM-CaSSIS Compatible" $\left(\mathrm{C}^{4}\right)$ cube, and are 250 described in more detail below. A fully-simulated CaSSIS cube is produced by 251 merging one of the two previously mentioned simulated colour cubes $\left(C^{4}\right.$, but $C^{3}$ 252 if $\mathrm{C}^{4}$ not available) with a 32-bit radiometrically calibrated I/F panchromatic image 253 from CTX (Malin et al 2007) that is oversampled from $\sim 5-6 \mathrm{~m} /$ pixel to the pixel254 resolution of CaSSIS (i.e., $\sim 4.6 \mathrm{~m} /$ pixel). In only a few cases, resampled 32-bit 255 radiometrically calibrated I/F HiRISE RED mosaic images (McEwen et al 2007, 2010; 256 Delamere et al 2010) were used instead of CTX.

Triple or double coordinated MRO observations (i.e., simultaneous 258 acquisition of CRISM-CTX, CRISM-HiRISE, or CRISM-CTX-HiRISE) are sought to 
259 mitigate issues inherent in observations taken at different times with disparate 260 observation geometries and acquisition circumstances. MRO coordinated 261 observations are ideal for CaSSIS simulated data product generation as they share 262 a common atmospheric state (weather conditions), sun-surface-spacecraft 263 geometry (incidence, emission and phase angles), season $\left(L_{s}\right)$, and no surface 264 changes in areas of Mars known to have seasonal changes. In cases where 265 coordinated observations were unavailable, observations taken at different times 266 were used, but only where surface changes, weather, and geometric discrepancies 267 were deemed to be minimal These simulations were kept to a minimum (only 4 268 cases, see Table 2), as coordinated observations are strongly preferred for the best 269 simulated results.

270 Also in a few cases, and were CTX and HiRISE stereo-derived DTMs were 271 available, we resampled these DTMs to $18.4 \mathrm{~m}$ post (i.e., $3 x$ the anticipated pixel272 scale). For one simulated test case, specifically geared toward assessing spatial 273 changes in the Nili Patera active dune field, a time-series pair of CTX images were 274 controlled to a HiRISE stereo pair and a pre-existing Digital Terrain Model (DTM). 275 For this task, SOCET SET ${ }^{\circledR}$ BAE system photogrammetry software and the 276 techniques summarized by Kirk et al (2008) were employed. Orthorectified CTX 277 images were oversampled to the CaSSIS pixel-scale $(4.6 \mathrm{~m} / \mathrm{pixel})$, and then change 278 detection methods, including animated GIFs and difference maps were applied to 279 see if spatial changes could be detected (see Section 3.5.2 for more details).

280 Radiometrically calibrated Planetary Data System (PDS) datasets for 281 CRISM, CTX and HiRISE were used in this study with all general processing tasks 282 described herein performed with a software package from Harris Geospatial 283 Solutions that includes both Environment for Visualizing Images (ENVI) and the 284 Interactive Data Language (IDL). Integrated Software for Imagers and 285 Spectrometers (ISIS) pre-processing of 32-bit CTX I/F data was accomplished and 286 obtained through the USGS PILOT web interface. Gain/offsets to convert 287 calibrated 8-bit HiRISE images to 32-bit I/F data was accomplished in ENVI via the 288 HiRISE Toolkit available through the PDS. 
The spectral/colour component of a given CaSSIS simulation is derived from

291 a CRISM hyperspectral targeted observation VNIR image cube (VNIR S-detector:

292 364-1055 nm; $6.55 \mathrm{~nm}$ sampling; 20 m/pixel full spatial resolution) (Murchie et al

293 2007). The spectral sampling characteristics of the CRISM instrument allow for the

294 synthesis of CRISM-derived data products that are spectrally compatible with

295 other Martian remote sensing instruments (Seelos et al 2011a), such as CaSSIS

296 (Thomas et al this issue) and HiRISE (McEwen et al 2007).

297 The "CRISM-compatible" data product concept originated as part of an 298 ongoing CRISM/HIRISE inter-instrument calibration effort, which has also been 299 employed in CRISM/HiRISE radiometric data fusion efforts (e.g., Seelos et al $3002011 \mathrm{~b}])$. An overview of the CRISM-HIRISE compatible transform is included as part 301 of our supporting online supplemental materials included with this manuscript 302 (also refer to Figures S1-S3), with the specifically illustrated in Figure S4, and 303 provided as a detailed walkthrough using ENVI/IDL therein.

The transformation process is fundamentally similar to the CRISM-HiRISE 305 one, with the four CaSSIS instrument response functions (BLU, PAN, RED, NIR) 306 taking the place of the three HiRISE response functions (BG, RED, IR) (c.f., Figure 2 307 with Figure S1). The "CRISM-CaSSIS Compatible" $\left(C^{3}\right)$ spectral processing portion 308 of the transformation of a CRISM VNIR image cube consists of the following steps, 309 and is illustrated in Figures $3 a$ and $3 b: 1$ ) Transforming the CRISM reflectance (I/F) 310 image cube to the equivalent spectral radiance; 2) Interpolating the radiance 311 spectra across the CRISM VNIR filter boundary ( $\sim 638 \mathrm{~nm}-\sim 690 \mathrm{~nm})$ to cover the 312 CaSSIS PAN bandpass filter, extrapolating the spectra short-ward of $\sim 410 \mathrm{~nm}$, and 313 long-ward of $1017 \mathrm{~nm}$ as required to cover the full wavelength ranges of CRISM 314 and CaSSIS (for the CaSSIS BLU and NIR bandpass filters, respectively); 3) 315 Oversampling the interpolated/extrapolated radiance spectra to match the 316 spectral sampling of the compatible instrument response functions ( $1 \mathrm{~nm}$ sampling 317 of the CaSSIS response curves); 4) Integrating the interpolated/extrapolated and 318 oversampled radiance spectra with respect to the instrument response functions; 319 and 5) Transforming the result back to I/F.

\section{0 [FIGURE 3]}


The resulting data product inherits the spatial and geometric

322 characteristics and radiometric calibration from the input CRISM image cube, but

323 has the spectral characteristics of the CaSSIS instrument. We note that the $\mathrm{C}^{3}$ data

324 products consist of transforming the as-acquired top-of-atmosphere (TOA) CRISM

325 spectral reflectance (Figure 3a) to the compatible CRISM-CaSSIS 4-band

326 reflectance without any additional data processing or corrections. As a result, the

$327 \mathrm{C}^{3}$ image cubes retain spectral-photometric variations inherent to the CRISM

328 hyperspectral targeted observation data acquisition scenario, specifically the

329 continuously varying observation geometry due to the requisite gimbal motion

330 required for targeted CRISM imaging mode. The varying viewing geometry of

331 CRISM targeted observations typically manifests as a wavelength-dependent

332 along-track gradient with brightening toward the inbound and/or outbound edge

333 of the observation central scan (c.f., Figure $3 a$ and $3 b$ with $3 c$ and $3 d$ ). These signal

334 gradients are due primarily to the change in atmospheric path length, and the

335 strong phase angle and wavelength dependence (over the CRISM VNIR and CaSSIS

336 wavelength range) of atmospheric aerosol scattering (Wolff et al 2009), but also

337 include some contributions from surface material scattering from these

338 observation geometries as well (e.g., Fernando et al 2016).

339 Although CaSSIS will acquire along-track stereo observations at different

340 phase angles, the CRISM continuous gimbal motion is not consistent with CaSSIS

341 push-frame (or HiRISE pushbroom) data acquisition. Therefore, to address this

342 difference in how CRISM and CaSSIS image Mars, an additional data processing

343 procedure is employed that results in data products which are consistent with the

344 CaSSIS data acquisition scenario. We refer to this final spectral/colour data product

345 as a "Corrected CRISM-CaSSIS Compatible" $\left(C^{4}\right)$ image cube. These products

346 incorporate an empirical CRISM geometric correction that transforms the original

347 CRISM image cube (Figure 3a) to a synthetic state as though the data were

348 acquired in a push-broom geometry (Figure 3c). Then the corrected CRISM image

349 cube is transformed into the $\mathrm{C}^{4}$ data product (Figure $3 \mathrm{~d}$ ) by the CRISM-CaSSIS

350 compatible spectral processing as described above. The Empirical Geometric

351 Normalization (EGN) procedure (Seelos et al 2011a) is a key component of the

352 CRISM Targeted Empirical Record (TER) / Map-projected Targeted Reduced Data 
353 Record (MTRDR) data processing pipeline (Seelos et al 2012; 2016), which

354 characterises the geometric dependencies across all segments of a CRISM targeted

355 observation, including the central scan and all available accompanying emission

356 phase function (EPF) segments, and then scales the dependencies out of the

357 central scan data relative to a reference observation geometry. We note that the

$358 \mathrm{C}^{4}$ data products do retain both the photometric geometry (incidence, emission,

359 phase angle) of the source CRISM observation at its corresponding reference

360 geometry, and the combined effects of the surface/atmosphere system. As a

361 result, the $C^{4}$ products have spectral/colour variability consistent with as-acquired

362 TOA CaSSIS image cube. Importantly, the CRISM EGN procedure is dependent on

363 the availability of EPF segments that sample more extreme emission angles than

364 the central scan. Unfortunately, at the beginning of October of 2012, the CRISM

365 gimbal range was restricted due to hardware ageing issues, and the acquisition of

366 EPFs were no longer possible. CaSSIS simulations that make use of CRISM targeted

367 observations acquired since the onset of gimbal range restrictions employ the $C^{3}$

368 products, and as such retain a non-pushbroom CRISM-source spectral-

369 photometric variability. However, the restricted gimbal range also limits the

370 emission and phase angle range sampled by latter-mission CRISM targeted

371 observations, which in turn can limit the geometric-dependent variability.

372 Therefore the $\mathrm{C}^{3}$-derived products can be employed on a case-by-case basis in the

373 evaluation of simulated CaSSIS intra-scene colour variability, particularly when

374 band ratios and other continuum-normalized evaluation criteria are considered,

375 and where it is not possible to generate $\mathrm{C}^{4}$ products.

376 2.2.2 Top of the Atmosphere (TOA) considerations - aerosol scattering in 377 the VNIR

378 The TOA spectral radiance measured by an orbital remote sensing 379 instrument is a function of the wavelength-dependent illumination source (the 380 solar spectral irradiance), surface spectral bidirectional reflectance, and 381 atmospheric radiative transfer. Over the CRISM VNIR and CaSSIS wavelength range 382 Mars atmospheric radiative transfer is dominated by dust (i.e., a ferric oxide 383 spectral signature) and water ice aerosols. Wolff et al (2009) derived the dust 384 aerosol radiative properties from 0.3 to 2.9 microns, in terms of the single 
385 scattering albedo [w], using CRISM EPF observations acquired during the 2007

386 global dust event. The dust particle single scattering albedo spectrum, which is the

387 fraction of incident light scattered by a single dust particle as a function of 388 wavelength, shows strong spectral variation directly related to the dust 389 composition (i.e., absorption coefficient). Dust aerosols scatter more light at longer 390 wavelengths $(w(700 \mathrm{~nm}) \sim 0.97)$ than at shorter wavelengths $(w(500 \mathrm{~nm}) \sim 0.82)$ 391 (see Figure 12 in Wolff et al 2009). There is also a shallow decrease in the dust 392 single scattering albedo with wavelength long-ward of $700 \mathrm{~nm}$ directly related to 393 an increase of the dust aerosol absorption coefficient (see Figures 12 and 13 in 394 Wolff et al 2009). The angular distribution of the light scattering from dust aerosols $395[p(g)]$ is anisotropic (e.g., Ockert-Bell et al 1997) and consequently the observed 396 scattering also depends on observation geometries. The direct consequence of the 397 dust aerosol scattering contribution in the VNIR range is a reddening of surface 398 signatures (i.e., impose a ferric absorption), which can influence the calculation of 399 surface spectral properties such as albedo and colour ratios from TOA spectra (see 400 Figure 1 in Fernando et al 2017). This effect is governed by the quantity of dust in

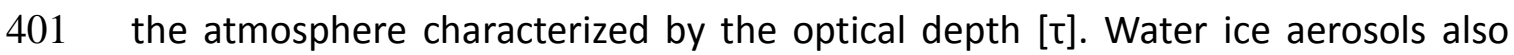
402 moderate the surface signal but the estimation of the radiative effect on a given 403 observation is complicated by their heterogeneous spatial and vertical 404 distributions in the Martian atmosphere as a function of time.

405 CRISM observations used for the "fully"-simulated CaSSIS effort in this 406 study were acquired with varying, but generally with favourable atmospheric 407 conditions (see Table 2). The subset of observations with a high atmospheric dust 408 and/or water ice optical depth ( $\tau_{\text {dust }}>1.0 @ 900$ nm (Wolff et al 2009 ); $\tau_{\text {ice }}>0.2 @$ $409320 \mathrm{~nm}$ (derived from MRO/MARCl data (Wolff, personal communication) must be 410 used with caution, particularly when relating TOA spectral properties (e.g., albedo, 411 and band ratios and spectral parameters) to corresponding calculations applied to 412 library spectra. In the CaSSIS data processing workflow, the EGN procedure 413 accommodates the aerosol scattering anisotropy, but it is a TOA relative correction 414 and so the $\mathrm{C}^{4}$ data products have not been corrected to ground reflectance. 415 Despite this, the variability in the $\mathrm{C}^{4}$ and the fully-simulated CaSSIS data products 416 (described in the next section) does accurately track intra-scene spectral/colour 
417 surface variability (as opposed to atmospheric or photometric effects. However,

418 due to the TOA nature of these products, an exact agreement with known mineral

419 and phase spectral libraries is not generally expected. The magnitude of the

420 surface vs. TOA discrepancy is directly governed by the atmospheric optical depth,

421 and so is minimised in CRISM scenes acquired when the atmosphere is relatively

422 clear (e.g., FRT00003E12; see Table 2).

\subsubsection{Creating a fully-simulated CaSSIS image cube}

The majority of the MRO instrument datasets from CRISM, CTX, and HiRISE PDS data products are not orthorectified, and the MRO instrument teams use

426 slightly different processing steps in the generation of map-projected products.

427 Consequently, even MRO coordinated datasets do not overlay one another 428 precisely. Therefore, image-to-image registration using a network of Ground

429 Control Points (GCPs) must be used to co-register the datasets before they can be 430 combined. This step is often an iterative process, if the topography of the scene is 431 complicated.

432 Overall, GCP-based warping of CRISM-derived $\mathrm{C}^{4}$ image cubes to 32-bit CTX

433 (or HiRISE) calibrated I/F images resampled to $4.6 \mathrm{~m} /$ pixel works well, especially 434 for images with minimal topographic variability. During processing, we noticed 435 that images with significant local-scale topographic variation were difficult to 436 align. Therefore, iterative steps of addition and adjustment of GCPs between the 437 CRISM-derived $\mathrm{C}^{4}$ cube and the corresponding high-resolution panchromatic base 438 image (i.e., 32-bit CTX or HiRISE I/F) was necessary to improve alignment. After the 439 initial selection of GCPs and warping, the warped $\mathrm{C}^{4}$ image is carefully compared 440 with the base image. This facilitates the identification of locations in the image 441 that require adjustment to, or additional, GCPs to minimise 442 misalignment/distortions and maximise the spatial pixel-to-pixel accuracy of the 443 fused transformed and spatially resampled MRO datasets into a final fully444 simulated CaSSIS image cube. This adjustment/addition of GCPs is followed by re445 warping the original $\mathrm{C}^{4}$ cube. This process can be repeated until the 446 misalignment/distortion between the two datasets is minimised. Sufficiently 447 accurate spatial co-registration is generally achieved after defining 75-100 well448 distributed GCPs for images with high-frequency topographic variability. 
Once the CRISM-derived $\mathrm{C}^{4}$ colour products are controlled to the spatially

450 rescaled CTX (or HiRISE) image, a pan-sharpening method is used to combine them

451 into a single cube. Pan-sharpening is a type of ground data processing and

452 spacecraft data-volume/bandwidth saving technique that merges a higher spatial

453 resolution grayscale panchromatic image with lower spatial resolution spectral

454 images to create a higher-resolution colour image or multispectral image cube

455 (e.g., McEwen and Soderblom 1984). The Gram-Schmidt spectral sharpening

456 transform (Laben and Bower 2000) is one of the most preferred pan-sharpening

457 methods used for terrestrial datasets, specifically VNIR, and is employed here to

458 create our final fully-simulated 4-band "CaSSIS" multispectral cube. Unlike many

459 of the other pan-sharpening methods used today (e.g., Hue-Saturation-Value -

$460 \mathrm{HSV}$ ), the Gram-Schmidt transform is a spectral pan-sharpening transform that

461 retains the radiometrically calibrated I/F values of the input images while

462 maximizing colour sharpness and keeping colour distortion to a minimum (Mauer

463 2013). The transform first simulates the panchromatic band or image (i.e., CTX or

464 HiRISE greyscale images) based on the lower spatial resolution spectral bands or

465 image cube (i.e., the CRISM-derived $\mathrm{C}^{3}$ or $\mathrm{C}^{4}$ products). Next, this lower spatial

466 resolution simulated panchromatic band is used as the first band in a forward

467 Gram-Schmidt transform. The first transformed band is then swapped out for the

468 higher spatial resolution panchromatic band or image, and an inverse Gram-

469 Schmidt transform is applied to generate the pan-sharpened spectral bands. The

470 result is a spatially $(4.6 \mathrm{~m} / \mathrm{pixel})$ and spectrally (4-band) simulation of a CaSSIS

471 image cube. Note that we calculate an additional CaSSIS band that we denote as

472 "band 0" (see Table 1). This is a synthesized "blue" wavelength band, which is

473 calculated in a similar fashion to the synthetic "blue" generated for HiRISE RGB

474 images by using the HiRISE "BG" and "RED" bands (Delamere et al 2010). In the

475 case of CaSSIS, the synthesized blue band is generated from the BLU and PAN

476 bands. A detailed walkthrough of the various techniques used here to generate

477 our fully-simulated CaSSIS image cube in ENVI/IDL is provided as part of our

478 supporting online supplemental materials.

479 Individual CRISM VNIR full spatial resolution spectral bands have a SNR in

480 the range of $\sim 100$ to $\sim 500$. The $C^{3}$ and $C^{4}$ data products integrate numerous CRISM 
481 channels in the calculation of the compatible CaSSIS spectral bands. As a result,

482 the $C^{3}$ and $C^{4}$ products have a significantly higher SNR (c.f., Figures $3 a$ and $3 b$ with

483 Figures $3 c$ and $3 d$ ). Although a quantitative calculation of the SNR of fully-

484 simulated CaSSIS image cubes have yet to be determined, it is likely that their SNR

485 are marginally higher than what will be observed with real CaSSIS images. We also

486 note that the SNR for the CaSSIS PAN band will be significantly higher than CTX (for

487 a surface at comparable lighting and atmospheric conditions) and possibly for

488 HiRISE as well (see Thomas et al and Roloff et al this issue).

\subsubsection{Simulated CaSSIS image site selection}

The Viviano-Beck et al (2014) study presents a summary of 31 type-spectra

491 of minerals and phases on Mars using CRISM data and which have been compiled

492 into the MICA (Minerals Identified through CRISM Analysis) spectral library. These

49331 CRISM spectral type-localities are covered by 27 individual CRISM hyperspectral

494 targeted observations (see Table 1 in Viviano-Beck et al [2014] for the specific

495 mineral or phase, image ID, and associated reference(s), location, and

496 corresponding best-matching analogue lab-measured mineral spectra).

\section{7 [FIGURE 4]}

498 In addition to these type-localities, coordinated MRO observations 499 covering key sites that fall under the defined science theme groups of the CaSSIS

500 team were selected for simulation, for a total of 181 simulated locations 501 including Phobos (Figure 4). The resulting set of simulated images generated for

502 this paper is by no means comprehensive with respect to all the science themes

503 and the many ongoing investigations of surface processes on Mars currently being

504 conducted by other moderate- to high-resolution imagers (e.g., HiRISE, CTX, HRSC

505 and MOC). However, these set of simulations should provide ample examples

506 demonstrating the anticipated colour and spatial capabilities of CaSSIS in the

507 context of a few selected key investigations. Preference was given to sites and

508 investigations where the colour and spatial abilities of CaSSIS need to be further

509 tested (e.g., resolving small scale features studied by HiRISE, such as Recurring

510 Slope Lineae) and where it has been speculated that the extended colour

511 capabilities would be particularly useful. The simulations provided here are meant

512 to provide an idea of the strengths/limitations of the CaSSIS dataset returned 
513 during the primary science phase and beyond, and to what extent CaSSIS will

514 compliment and extend existing or concurrent datasets.

515 Lastly, we specifically note here that while some actual CaSSIS images have

516 been acquired during a series of Mars Capture Orbit (MCO) imaging campaigns in

517 November 2016 and February 2017 (see Pommerol et al 2016), and that some

518 comparisons between simulated CaSSIS products and actual images are underway

519 (Pajola et al 2017a), actual image radiometric and geometric calibration efforts are

520 currently in progress (e.g., Tulyakov et al 2017), and therefore the comparisons

521 are beyond the scope of this paper. Furthermore, actual CaSISS images taken

522 during MCO where taken on highly elliptical orbits, which do not provide a good

523 test of the spatial capabilities of CaSSIS at its anticipated pixel-scale resolution.

\section{Results}

\section{$525 \quad 3.1$ Fe-sensitivity with CaSSIS colour image products}

526 Lab-derived spectra for forty-three ferrous iron-bearing, including mafic

527 minerals, and fourteen ferric iron-bearing minerals were resampled to 4-point 528 CaSSIS-convolved spectra (i.e., BLU, PAN, RED and NIR). The hyperspectral lab

529 spectra and CaSSIS-convolved spectra (e.g., Figure 1) can be visually compared to

530 one another to understand the spectral sensitivity that remains after convolution

531 to the 4 bands of CaSSIS from the hundreds of bands of the lab spectra that cover

532 the CaSSIS sensitivity range ( $400-1100 \mathrm{~nm}$ ). Referring back to Figure 1, we can

533 observe that CaSSIS-convolved spectra for ferrous iron-bearing olivine and ferric

534 iron-bearing nontronite are still quite distinguishable from one another, exhibiting

535 strong spectral slopes toward the major absorption features associated with the

536 presence of ferrous and ferric iron in these two example minerals, respectively.

537 While additional comparisons of ferrous iron- (e.g., pyroxenes) and ferric iron-

538 bearing (e.g., hematite, goethite, etc.) mineral/phase spectra show that it is not

539 generally possible to identify a mineral or phase uniquely from 4-point CaSSIS

540 spectra alone, it is nonetheless possible to distinguish ferrous iron- and ferric iron-

541 bearing species from each other rather consistently.

\section{2 [FIGURE 5]}


This is further exemplified by plotting the CaSSIS-convolved ratioed band

544 values based on multiple mineral/phase spectra on bivariate diagrams (e.g., 545 McEwen et al 2007; Delamere et al 2010), and provides another means of 546 assessing the Fe-sensitivity of CaSSIS without having to plot and compare 547 numerous spectra (Figure 5). The plots in Figure 5 show that Fe-bearing minerals 548 behave in a predictable and consistent manner as a function of the CaSSIS band 549 sensitivity to the known locations of absorptions, or a lack thereof. For example, a 550 PAN/BLU ratio is used to highlight minerals and phases with a strong ferric iron $551\left(\mathrm{Fe}^{3+}\right)$ absorption short of $550 \mathrm{~nm}$ (e.g., Figure 1) by producing higher ratio values

552 for minerals and phases that are dominated by ferric iron over ferrous iron $\left(\mathrm{Fe}^{2+}\right)$. 553 We observe an expected greater spread in the value of ferric iron-bearing minerals 554 and phases (mean: 2.11 std. dev.: 0.66) for this ratio because the absorption 555 feature depth for ferric iron varies due to several factors (e.g., mineral 556 composition, grain size, etc.), whereas we see very little spread (mean: 1.11 std. 557 dev.: 0.11) for the values for ferrous iron-bearing minerals and phases that 558 generally lack an absorption feature short of $550 \mathrm{~nm}$. Likewise, we see similar good 559 discrimination behaviours with respect to the PAN/NIR ratio values, which is 560 designed for highlighting minerals and phases with a strong ferrous iron $\left(\mathrm{Fe}^{2+}\right)$ 561 absorption near $1000 \mathrm{~nm}$, when comparing ferrous iron- vs. ferric iron-bearing 562 minerals and phases.

563 Our analysis of the 57 CaSsIS-convolved lab-collected mineral and phase 564 spectra shows that the four CaSSIS colour bands will provide ample sensitivity to 565 Fe-bearing minerals that will rival the ability of HiRISE. This is due to the better 566 SNR of CaSSIS and the splitting of the HIRISE NIR band into two IR bands for CaSSIS 567 (i.e., RED and NIR; see Table 1). This two NIR bands allow for an additional band 568 ratio to be formulated (i.e., RED/PAN) over HiRISE (Delamere et al 2010). The plots 569 in Figure 5 and Table 3 provide a synthesis of the results and illustrate the most 570 useful band ratio combinations for CaSSIS images, which we can use to achieve 571 the best visual discrimination among the various Fe-bearing surface materials 572 known to exist on Mars. To summarize, a PAN/BLU ratio (675 nm / $500 \mathrm{~nm}$ ) 573 provides the strongest discrimination between ferric iron- and ferrous iron- 
574 bearing minerals followed by PAN/NIR ( $675 \mathrm{~nm} / 937 \mathrm{~nm}$ ), and RED/PAN (836 nm

$575 / 675 \mathrm{~nm})$.

576

577

578

579

580

581

582

583

584

585

586

587

588

\subsection{Ice/Frost and/or Atmospheric Phenomena sensitivity with CaSSIS colour image products}

Although Figure 1 shows that CaSSIS should have some sensitivity to the absorption around $1030 \mathrm{~nm}$ due to the presence of ice/frost, the broadband nature of the 4 CaSSIS bands, and the fact that ferrous iron-bearing phases $\left(\mathrm{Fe}^{2+}\right)$ have a stronger absorption around $1000 \mathrm{~nm}$, poses issues for creating a CaSSISderived band ratio or spectral parameter that will readily distinguish pixels that are influenced ice/frost. Basically, a ratio that relies on the band depth of the NIR band will more readily distinguish any ferrous iron-bearing materials in any given scene over $\mathrm{H}_{2} \mathrm{O}$ frost/ice. Therefore, any simple ratio using the NIR band will not unambiguously isolate $\mathrm{H}_{2} \mathrm{O}$ frost/ice in a scene that contains any ferrous iron component.

\section{[FIGURE 6]}

Based on previous methods, single wavelength blue or blue-green image and/or a red- or NIR-to-blue ratio (e.g., James et al 1994; Bell et al 1997; Pelkey et al 2007; Murchie et al 2009; Viviano-Beck et al 2014) have been used with some success to isolate pixels containing a signature of ice/frost and/or atmospheric phenomena in a given image (e.g., see Figure 6). This is because the Martian dust, which covers most of the surface, is bright, red and lacks blue due to a broad ferric $\left(\mathrm{Fe}^{3+}\right)$ iron absorption over the blue wavelengths; whereas, ices and atmospheric phenomena are also very bright, they do not generally lack blue (Figure 6a) (except perhaps in cases where ice may be contaminated with Martian dust). The remaining Martian surfaces that lack both dust and ice are generally bluer because they generally lack ferric $\left(\mathrm{Fe}^{3+}\right)$ iron and possess a ferrous $\left(\mathrm{Fe}^{2+}\right)$ iron component. However, similar to the discussion above, a simple red-or NIR-to-blue ratio alone to isolate the signatures of ices or atmospheric phenomena will be confounded in scenes where both materials are present (e.g., Figure 6b). However, we note that these dust- and ice-free surfaces have the very lowest reflectance or albedo values on Mars (e.g., Syrtis Major). This suggests that a multiband mean reflectance or I/F intensity image (as a proxy for albedo) may be used to aid discrimination of 
606 pixels influences by ice/frost and/or atmospheric phenomena. Unfortunately,

607 natural variations in topography and observation geometries at high-resolution

608 are an issue in a multiband mean reflectance or I/F intensity image as sun-lit light-

609 toned bedrock and Martian dust-bearing surfaces may be just as bright as ice/frost

610 and atmospheric phenomena (e.g., Figure 6c). While a single band blue or blue-

611 green image compared to a colour R-G-B image does a fairly good job at

612 distinguishing ice/frost and atmospheric phenomena, a single band image will still

613 contain illumination effects, which may cause some ambiguity and slow down

614 analysis (c.f., Figure 6a and 6d).

Although we find that each of the different suggestions summarized above cannot be used alone to readily and unambiguously distinguish ice/frost or

617 atmospheric phenomena in images, we have determined a simulated CaSSIS

618 spectral parameter that combines the individual strengths into a single spectral

619 parameter that works exceptionally well, especially when compared to CRISM IR

620 hyperspectral parameters for ices (Pelkey et al 2007; Murchie et al 2009; Viviano-

621 Beck et al 2014) (c.f., Figures 6e and 6f). The spectral parameter is created by

622 dividing a mean multiband reflectance or I/F intensity image by a red- or NIR-to-

623 blue ratio (e.g., NIR/BLU, RED/BLU or PAN/BLU) image, and applying a min/max

624 linear stretch (e.g., Figure 6e). Figure 6e shows that the illumination effects are

625 minimised and that the spectral parameter provides an excellent separation of

626 ice/frost and atmospheric phenomena from other Martian surface components

627 that is better than just a single wavelength blue or blue-green image alone (c.f.,

628 Figures 6 e and 6a). This separation also appears to be possible with a two-band 629 image, one that includes the mean of the blue-green (BLU) band and at least one 630 other CaSSIS band (PAN, RED or NIR), divided by a ratio of the two.

$631 \quad 3.3$ Suggestions for CaSSIS standard colour and band ratio colour 632 composite image products

633 One hundred and eighty-one $C^{3}$ products were generated in this study, and 634 of these 18 were not suitable to be processed as $C^{4}$ products (i.e., CaSSIS colour635 compatible products with the CRISM gimbaled/variable imaging geometry 636 normalized to a reference observation geometry). As previously discussed in 637 Section 2.2.1, this is due to an insufficient number of EPF segments associated 
638 with a given CRISM targeted observations (i.e., less than five). This also includes

639 the CRISM observation of Phobos (FRT00002992), which, despite the 'FRT' class

640 type, was acquired in a manner inconsistent with the geometric normalization

641 procedure. A total of 33 "fully" simulated CaSSIS image cubes were generated

642 (Table 2) based on $C^{4}$ products with the exception of one - the Mars Science Lab

643 (MSL) "Curiosity" landing site (FRS0028346). Only a $\mathrm{C}^{3}$ product was possible for

644 this site as no EPF segments were acquired in association with this

645 CRISM targeted observation taken in early 2013. The 33 fully-simulated CaSSIS

646 cubes cover 30 unique locations on the Martian surface, as six of the cubes

647 represent change detection pairs for the "Inca City", "Arrakis" and Gasa crater

648 sites. A database/table (Table S1) including information on the 181 CRISM

649 observations is included in three formats (.csv, .xlsx and ESRI shapefile .shp) as part

650 of our supporting online supplementary materials, please see these resources and

651 the associated help file for more information on the observations used to generate

652 our CaSSIS simulated products.

653 Standard colour RGB band combination images, and Colour Band Ratio

654 Composites (CBRCs) for all simulated products were created as another tool to

655 assess the colour capability of CaSSIS towards differentiating various surface and

656 atmospheric components. With two near-infrared CaSSIS bands to choose from

657 (Table 1), we can create two IRB (i.e., infrared-red-blue) infrared colour images,

658 which we refer to here as IRB1 and IRB2; these combinations correspond

659 specifically to simulated CaSSIS band combinations of 4-2-1 or NIR-PAN-BLU and

$6603-2-1$ or RED-PAN-BLU in the R-G-B channels, respectively. The synthesized blue

661 wavelength band (i.e., band 0) provides the means to produce a synthetic "true"

662 colour composite image that is similar to the HiRISE RGB (red-green-blue) product,

663 which is created with simulated CaSSIS products by combining the 2-1-0, or PAN-

664 BLU-"Blue", bands in R-G-B channels. Based on the results of our analysis of lab

665 spectra convolved to the CaSSIS instrument response functions, a CBRC

666 combination that combines simulated CaSSIS band ratios RED/PAN, PAN/BLU and

667 PAN/NIR in the R-G-B channels (we refer to this product as CBRC1) to maximize

668 the distinction of Fe-bearing minerals and phases; while for distinguishing ice/frost

669 or atmospheric phenomena and other surface materials, we recommend a CBRC 
670 combination of PAN/BLU, PAN/NIR and Mean[BLU-NIR]/(NIR/BLU) in the R-G-B

671 channels (we refer to this as CBRC2). Our analysis also indicates that a CBRC2

672 image that uses just the BLU band in the blue channel does an adequate job of

673 distinguishing ice/frost or atmospheric phenomena from other surface materials,

674 but the former spectral parameter provides better separation when available. All

675 standard R-G-B images have a default 0.5\% linear stretch applied to them, unless

676 stated otherwise.

677 Figure 7 shows an example set of simulated CaSSIS colour image products 678 based on CRISM observation FRT00003E12, which is the same CRISM observation 679 that was used by Delamere et al (2010) as an example location for the 680 effectiveness of HiRISE colour products to extend hyper- and multi-spectral 681 datasets (e.g., CRISM and THEMIS, respectively). As such, this is also an excellent 682 example location for assessing both HiRISE and CaSSIS colour capabilities, as the 683 Nili Fossae region of Mars is known for its mineral diversity and particularly for 684 both spatially separated and distinctive ferrous- and ferric-bearing surface units. 685 The CBRC1 combination employed here uses all four CaSSIS colour bands and is 686 noted for providing extra sensitivity and discrimination of altered, ferric iron687 bearing materials when compared to the band ratios used for a HiRISE CBRC image 688 combination presented in Delamere et al (2010). The enhanced colour diversity 689 and sensitivity brought out by the simulated CaSSIS CBRC1 image over the HiRISE 690 CBRC is readily observed by comparing the one provided in Figure 7 with Figure 12 691 in Delamere et al (2010) (also see an additional example included in the 692 Composition and Photometry section - section 3.5.1 - which covers the reported 693 CRISM type-locality for the mineral talc). Furthermore, single-band ratio images 694 that use a colour table to highlight pixels with the greatest band strength (bottom 695 row of Figure 7), will be extremely useful as a proxy for exposure/concentration 696 maps that may be utilized for extending orbital mineral mapping, but more 697 importantly as a in situ exploration guide for landed missions.

\section{8 [FIGURE 7]}

699 The Fe-sensitivity of CBRC1 is not only supported by the above example, 700 but by also analysing image-derived spectra extracted from the 4-band $C^{4}$ image 701 cube based on CRISM FRT00003E12 (Figure 8). Here we extracted spectra for 
702 coloured spectral units based on our CBRC1 product (see Figure 7) both before

703 and after implementing a dark subtraction method in ENVI to mitigate issues with

704 wavelength-dependent atmospheric scattering in each of the 4 bands. Despite the

705 TOA nature of the image-derived spectra, the spectra extracted from the $C^{4}$ cube

706 have the general shapes, slopes, and features expected based on the known

707 spectral units and mineral matches for this location on Mars (see Mustard et al

708 2007, 2009; Tornabene et al 2008; Ehlmann et al 2008, 2009; Delamere et al 2010)

709 and when compared to lab-derived spectra. The FRT00003E12 $\mathrm{C}^{4}$ image derived

710 spectra for Fe/Mg-bearing olivines and smectites are similar to the CaSSIS

711 convolved lab-derived spectra shown (c.f., Figure 7 and 1). However, this is only

712 because there are minimal effects from dust and water-ice atmospheric

713 contributions for these TOA spectra (see Table 2 and Figure 1 in Fernando et al

714 [2017] for more details).

715 [FIGURE 8]

$716 \quad 3.4$ General results based on $\mathrm{C}^{4}$ CaSSIS colour products and fully717 simulated CaSSIS image cubes

718 Our 33 fully-simulated 5-band image cubes currently include 4 of 27 CRISM

719 images covering the spectral mineral/phase type-localities presented in Viviano-

720 Beck et al (2014), specifically for olivine/Mg-carbonate, talc, kaolinte, and low-

721 calcium pyroxene (LCP).

722 [FIGURE 9]

723 Figure 9 shows a compilation of CBRC1s generated exclusively from

724 simulated $C^{4}$ CaSSIS colour products based on 24 of the 27 CRISM images covering

725 the spectral mineral/phase type-localities. Notably, the most diversely coloured

726 CBRC1 images in the set (Figure 7 and Figures 9d, 9e, 9l-n, 9p, 9s-x) correspond to

727 mineral localities or locations on Mars that contain excellent exposures of

728 widespread Fe-bearing minerals and phases.

729 In general, the CBRC1 shows ferrous iron-bearing minerals in blue and

730 ferric iron-bearing materials in yellow and orange. Although many of these

731 localities show diverse colours consistent with abundant Fe-bearing minerals and

732 phases, there are a few colour-diverse CBRC1 images shown in Figure 9 that show

733 similar colour diversity that in some cases are actually due to a lack of spectral

734 contrast (red coloured unit; also see spectrum in Figure 8) or a relative lack of Fe-

735 bearing phases. In such cases, the lack of Fe-bearing materials results in minimal 
736 contributions to the blue and green channels of the CBRC1 image; this results in a

737 strongly red or reddish-orange coloured unit. Examples of this include some

738 occurrences of Al-bearing smectites (Figure 9m-n) and chloride-bearing deposits

739 (Figure 9x). An additional example of chlorides with a strong red and orange colour

740 in our CBRC1 product is provided in the Composition and Photometry section

741 below (section 3.5.1).

742 [FIGURE 10]

743 Figure 10 shows an example of an infrared colour image constructed from 744 fully-simulated, 5-band and $4.6 \mathrm{~m} /$ pixel CaSSIS image using bands 4-2-1 (or NIR745 PAN-BLU) in R-G-B. For those that are familiar with HiRISE colour images, the 746 simulated CaSSIS IRB appears remarkably similar in colour to the HiRISE IRB 747 products for this site (see HIRISE IRB for PSP_002176_2025). The fully-simulated 748 cube was cropped for the figure to demonstrate the two anticipated swath width 749 modes planned for CaSSIS imaging (full-width: 9.4 km; short-width: $7.1 \mathrm{~km}$ ) and 750 compared to the HiRISE full-swath width $(\sim 1.2 \mathrm{~km})$. Additional discussion of this 751 example is provided in the Composition and Photometry section (section 3.5.1).

752 Although DTMs are not a focus of this simulation study, HIRISE and CTX 753 DTMs (when available), were resampled to 18.4-m post-spacing, which is based 754 on a simple 3-pixel solution for deriving elevation from stereo images (e.g., Kirk et 755 al 2008). Figure 10 shows an example with the "mesh" function switched on in 756 ENVI; the mesh facilitates a comparison of the post-spacing pixel-scale of the DTM 757 in relation to surface/morphologic features. This illustrates a simple example of 758 what a 3D perspective using a CaSSIS colour image draped over a CaSSIS stereo759 derived DTM would look like, and how CaSSIS might provide constraints on the 760 geometry, thickness and 3D relationships (e.g., stratigraphy) of surface units, and 761 thereby the relative timing of their formation, possible origins and modification 762 histories.

$764 \quad 3.5$ Various examples of CaSSIS simulations by science theme

\subsubsection{Composition and Photometry}

Mars has a global surface composition that is more or less basaltic with a 
768 et al 2002); furthermore, the ice-free surfaces of Mars can be generally divided

769 into low albedo and high albedo surfaces, which strongly correlates with ferric

770 iron-rich dust coverage, where, in general, the higher the dust coverage of the

771 surface, the higher the albedo, and the redder the surface. Past and ongoing multi-

772 and hyper-spectral VNIR and thermal infrared (TIR) instruments currently in orbit

773 around Mars continue to provide more details into the mineral and phase

774 compositional diversity of the surface at regional and local scales. The diverse

775 minerals and phases of Mars can be generally categorized into primary/unaltered

776 (mafic) and secondary/altered, or into groups (e.g., Viviano-Beck et al 2014), such

777 as: 1) primary mafic minerals and iron oxides; 2) sulphates; 3) phyllosilicates; 4)

778 carbonates; and 5) halides and other silicates.

779 Although most of these groups were known to exist on Mars through the 780 study of Martian meteorites (e.g., McSween et al 2002), the first group to be 781 identified spectrally on Mars was the primary mafic (e.g., Adams 1968; McCord 782 and Westphal 1971; Martin et al. 1996) and iron oxides group minerals (e.g., 783 Adams and McCord 1969; McCord et al 1977, 1978; Singer et al 1979). Although 784 several minerals were suggested to exist on the Martian surface based on 785 telescopic observations (e.g., a ferric-bearing phase, pyroxenes, etc.), crystalline 786 grey hematite, a common ferric iron-bearing mineral, was spectrally isolated in the 787 Meridiani Planum region with hyper-spectral data from the Thermal Emission 788 Spectrometer (TES) on the Mars Global Surveyor (Christensen et al 2000). This was 789 followed by the isolation and identification of olivine spectra correlating to specific 790 Fo\# compositions (i.e., Fosterite/Fayalite content) near Nili Fossae and Isidis 791 Planitia (Hoefen et al 2003; Hamilton and Christensen 2005; Koeppen and 792 Hamilton 2008; Tornabene et al 2008); however, the verification of the presence 793 of other primary mafic minerals (e.g., low and high Ca-pyroxenes, and high Ca794 plagioclase) from orbit was based on the inclusion of these mineral spectra to 795 properly model and spectrally unmix TES surface spectra (e.g., Christensen et al 796 2000; Bandfield et al 2001). 798 phases, were in some cases inferred from the results of TES spectral analyses (e.g., 
799 Christensen et al 2001), but were not unambiguously identified until the advent of

800 higher spatial resolution spectrometers (e.g., THEMIS, OMEGA and CRISM). The

801 emergent view of the occurrence of phyllosilicates on Mars, although observed to

802 be concentrated in some specific regions of Mars, is that they are generally

803 observed to be widespread amongst the oldest and most heavily cratered terrains

804 on Mars (e.g., Wray et al 2008; Ehlmann et al 2011; Carter et al 2013; Ehlmann

805 and Edwards 2014). Whereas, concentrations of sulphates detected from orbit

806 occur in two major localities - the canyon system of Valles Marineris (e.g., Bishop

807 et al 2009; Roach et al 2009) and the northern hemisphere circumpolar dunes of

808 Olympia Undae (e.g., Langevin et al 2005; Calvin et al 2009); however, sulphates

809 are far more abundant and extensive on the surface of Mars based on the results

810 of surface investigations by Opportunity, Spirit, Phoenix and Curiosity (Christensen

811 et al 2003; Klingelhöfer et al 2003; Rieder et al 2003; Gellert et al 2006; Morris et

812 al 2006a, 2006b; Kounaves et al 2010; McLennen et al 2014). Chloride deposits

813 are observed to be scattered throughout the Noachian highlands of Mars

814 (Osterloo et al 2008; 2010), and are sometimes associated with phyllosilicate clays

815 (e.g., Glotch et al 2010). The synthesis provided by Ehlmann et al (2011) shows

816 that $\mathrm{Fe} / \mathrm{Mg}$ phyllosicate clays are the most abundant secondary/aqueous

817 alteration minerals identified, with Fe-poor minerals and phases generally being

818 the least abundant. This bodes well for CaSSIS as it will be most sensitive to the

819 Fe-bearing minerals and phases; however, the results of our simulations show that

820 some Fe-poor minerals can appear quite distinctive in colour, specifically in our

821 CBRC1 product. Due to a strong lack of absorptions from either ferrous or ferric

822 iron-bearing materials in some deposits, and certainly when they are juxtaposed

823 amongst a generally Fe-rich background, our CBRC1 product shows Fe-poor

824 minerals and phases, such as Al-bearing phyllosilicate clays and chloride deposits,

825 as strongly red or orange coloured units (also see Figures $9 \mathrm{~m}-\mathrm{n}$ and $9 \mathrm{x}$ ). Figure 11

826 shows an excellent example of the chloride-bearing deposits (reds and oranges)

827 reported by Osterloo et al (2008), which is observed to starkly contrast with

828 ferrous iron-bearing lavas and crater ejecta (blues). We not that their

829 distinctiveness in CBRC1 is non-unique, and thereby CaSSIS can only provide 
830 further context for such minerals in cases where they have already been identified

831 in other datasets (e.g., THEMIS, CRISM, etc.).

\section{2 [FIGURE 11]}

A synthesis of decades of spectral analyses now provides us not only with

834 the identification of key mineral and phase localities on the surface of Mars (see

835 Viviano-Beck et al 2014 and references therein), but also the locations of some of

836 the most spectral diverse regions on the planet (see recent summaries by EhImann

837 et al 2011; Carter et al 2013; EhImann and Edwards 2014). Amongst this body of

838 results, several surface features and regions stand out (e.g., impact craters,

839 channels, valleys/grabens, Valles Marineris, Mawrth Vallis, Nili Fossae region,

840 etc.). These features and locations are anticipated to be highly valued targets for

841 the CaSSIS Composition and Photometry science theme. Herein below, we provide

842 a few fully-simulated CaSSIS examples of some of these features, and from these

843 regions, to demonstrate the capabilities we can expect from CaSSIS for

844 augmenting compositional studies of the Martian surface.

\section{Complex Crater Central Uplifts}

846 Orbital observations continue to reveal the morphologic, spectral and

847 structural complexity of complex crater central uplifts. Although Valles Marineris

848 (Viviano-Beck et al 2016) and other valleys or channels on Mars (e.g., Wray et al

849 2016) provide some of the best subsurface bedrock exposures on Mars, they occur

850 over limited regions and the geometry of the exposures are not always conducive

851 to observation from above. The bedrock exposed in complex crater central

852 features, however, are uplifted, rotated (as much as $90^{\circ}$ ) and exposed at the

853 surface making them ideal for orbital observation from above. A study of over 200

854 observations of well-exposed central uplifts indicate that craters provide the most

855 globally widespread occurrence of bedrock exposures across Mars (e.g.,

856 Tornabene et al 2015). A study focusing on hundreds of complex crater uplift

857 observations revealed three distinct bedrock textural classes: 1) a fractured and

858 massive bedrock (FMB); 2) "mega"-brecciated bedrock (MBB); and 3) layered

859 bedrock (LB). Tornabene et al (2015) also summarized the types and stratigraphic

860 relationships of impact-related deposits and structures, including breccias, clast- 
861 rich and clast-poor melt-bearing deposits, dykes, and both faulting and what

862 appears to be folding observed primarily in layered uplifts (see Caudill et al 2012).

863 Several of the best-exposed and most spectrally diverse central uplifts on Mars

864 have been the focus of detailed studies (Marzo et al 2010; Osinski et al 2011;

865 Quantin et al 2012; Skok et al 2012; Wulf et al 2012; Sun and Milliken 2014, 2015;

866 Nuhn et al 2014, 2015; D’Aoust 2015; Ding et al 2015; Hopkins et al 2016) spanning

867 topics that include: 1) crustal formation and alteration history; 2) impact-induced

868 alteration mechanisms (including hydrothermal); and 3) central uplift formation,

869 and the timing and emplacement of impactite deposits. CaSSIS is anticipated to

870 augment these studies through its stereo and colour capabilities, especially colour

871 coverage, and thereby provide an improved understanding the various topics

872 mentioned above.

\section{3 [FIGURE 12]}

874 Figure 12 shows a summary figure of cropped portions of fully-simulated 875 CaSSIS infrared colour images covering the various bedrock texture classes 876 (Tornabene et al 2015) associated with crater central uplifts. All three bedrock 877 types, including the layered type (LB), are resolved by the simulated CaSSIS 878 images. Figure 13 shows additional details provided by a fully-simulated CaSSIS 879 infrared colour image that covers the central peak of Ritchey crater. These 880 simulation shows us the colour diversity, coverage, and rendered 3D perspective 881 views, that we might expect from CaSSIS. Impactites (e.g., impact melts and 882 breccias) and bedrock are readily resolved and recognized, in addition to their 883 stratigraphic relationships, thanks to the full-colour coverage and 3D perspective 884 provided by the CaSSIS simulation (c.f., 3D and 2D provided in Figure 13). Some 885 impact-related structures and features appear to be resolvable in these 886 simulations, but these features, such as crater-related dykes and smaller meter887 scale lithic clasts and certainly better resolved by HiRISE. This brings up an 888 important point. Where colour may be vital for detailed geologic mapping of such 889 small-scale features, and where HiRISE colour coverage may be lacking or difficult 890 to achieve, the use of CaSSIS colour images over these features can be pan891 sharpened using available HiRISE grayscale images. This is not only desirable, but 
892 highly recommended for not only impact-focused studies, but for other

893 investigations where contiguous high-resolution colour coverage is important

894 (e.g., landing site analysis, etc.). The examples provided in Figures 12 and 13

895 demonstrate how the increased colour coverage provided by the wider swath

896 width of CaSSIS will provide clearer, and in many cases, better context then HiRISE.

897 This is especially true where colour is vital towards identifying and mapping

898 distinct impact-related geologic features and their stratigraphic relationships, and

899 thereby improve our understating of the formation of the timing and formation of

900 said features. To truly optimize studies and our understanding of the geology of

901 complex crater central uplifts, we recommend combining CaSSIS DTM, spectral

902 and colour coverage information with HiRISE and other datasets, such as CRISM

903 and THEMIS-derived thermal inertia.

904 [FIGURE 13]

905 Possible methane producing regions on Mars

906 Focused studies of regions on Mars known to contain olivine and 907 associated alteration minerals and phases, particularly serpentine, will be of key 908 importance to the ExoMars 2016 TGO mission. Figure 7 and Figure 10 covers a 909 unique, morphologically and spectrally diverse location in the Nili Fossae region 910 that has been the focus of several intense studies that have made use for all 911 available VNIR and TIR spectrometer datasets collected from Mars orbit to date 912 (Hoefen et al 2003; Hamilton and Christensen 2005; Mustard et al 2007, 2008;

913 Tornabene et al 2008; Ehlmann et al 2009; Delamere et al 2010). This site includes

914 widespread olivine-bearing deposits (Hamilton et al 2003; Hoefen et al 2003;

915 Hamilton and Christensen 2005; Tornabene et al 2008) juxtaposed with Fe/Mg

916 smectite-bearing deposits (e.g., Mangold et al 2007; Mustard et al 2007, 2008;

917 Ehlmann et al 2009), Mg/Fe carbonates (Ehlmann et al 2008), and serpentine

918 (Ehlmann et al 2010). The presence of these minerals may be linked to methane

919 being produced as a geochemical by-product through the alteration of olivine-

920 bearing materials into serpentine (e.g., Wray and EhImann 2011; Viviano-Beck et

921 al 2013) (also see review by Holm et al 2015). This location is a relevant test case

922 for the ExoMars-TGO mission because it was identified as one of several possible

923 transient/seasonal methane source regions (Mumma et al 2009). The simulated 
924 CaSSIS images show the olivine-bearing, smectite-bearing, and carbonate-bearing 925 surface units as dark blue, orange/yellow, and greenish-yellow, respectively in the

926 CBRC1 image (Figure 7); and cyan, orange/yellow, light-cyan, respectively, in the

927 fully-simulated infrared colour image (Figure 10). Our example shows that the 928 mapping of these minerals detected by CRISM, will be readily extended by the

929 colour and spatial capabilities of CaSSIS by effectively providing a higher resolution

930 colour product and improved geologic context than CRISM or HiRISE colour could 931 provide alone. Colour mapping with HiRISE of this locality would require 932 mosaicking numerous narrow-swath HiRISE colour images, which are not easy to 933 obtain for large contiguous areas under similar observation geometries. Indeed, 934 the coverage required for HiRISE colour mosaicking for large areas are difficult to 935 plan and acquire and, hence has it seldom ever been done for even the highest 936 priority sites on Mars.

The study by Wray and Ehlmann (2011) also describe correlated 938 compositions and surface textures observed within the reported methane source 939 regions (Mumma et al 2009), including Nili Fossae. These regions are generally 940 comprised of older terrains cut by deep fractures and which do not appear to 941 include any evidence of younger volcanic activity. Many of the observed fractures 942 are likely ancient themselves, but a few are also found to be recent (as little as $943 \sim 10^{5}$ years?) as they postdate small aeolian bedforms (Wray and EhImann, 2011).

944 CaSSIS monitoring of these fractured terrains for changes may reveal candidate 945 sources for any trace gas activity that other instruments aboard TGO might detect.

946 The broader colour coverage of CaSSIS will undoubtedly aid in this task, as HiRISE 947 has shown that surface changes can often be seen much more readily in colour 948 data (e.g., McEwen et al 2010; also, see section 3.5.2 below). Although most 949 colour changes may reflect the removal and transport of surficial materials (dust, 950 sand), they may also indicate areas of new mineralization, which would be 951 expected from an active near-surface methane-generating serpentinization 952 processes.

953 Serpentine may be the most obvious mineral to look for in this context, but any 954 newly-fromed distinctly coloured aqueous minerals (e.g., Wray and Ehlmann, 
955 2011; Viviano-Beck et al 2013) may point to modern-day aqueous processes that 956 might relate to contemporaneous trace gas activity. The detection of talc or 957 chlorite may also be equally important, as discussed by Viviano-Beck et al (2013),

958 as the chloritization of smectites may have provided the silica-rich fluids necessary 959 for the serpentinization of olivine in the Nili Fossae area. Figure 14 shows a fully960 simulated CaSSIS product that covers the CRISM spectral mineral/phase type961 locality for the mineral talc. This compositionally diverse example again shows to 962 what extent the full 4-colour capability of CaSSIS will not only enable, but greatly 963 enhance detailed spectral mapping in this area. This example also demonstrates 964 the effectiveness of the CaSSIS CBRC1 that uses all 4 CaSSIS bands, including both 965 NIR bands, over a HiRISE-equivalent CBRC, which is limited to 3 bands and only a 966 single NIR band (Figure 14). The anticipated result from such detailed spectral 967 mapping augmented by CaSSIS colour/morphologic detail, will not only inform us 968 on the general geologic history of methane-source regions such as this, but also 969 place further constraints on the possible connection between surface/subsurface 970 mineral reactions that may be a primary source of methane on Mars today.

\section{1 [FIGURE 14]}

972 It is clearly demonstrated through our simulations that CaSSIS will be ideal 973 for documenting the compositional diversity of the Martian surface. This is due to 974 fact that the 4 bandpasses of CaSSIS are sensitive to the VNIR spectral range, and 975 that Mars hosts such a diversity of Fe-bearing minerals and phases, which produce 976 key absorptions in this range that are related to the iron oxidation state and the 977 coordination of iron in the specific mineral or phase. HiRISE has demonstrated 978 similar colour capabilities over the last decade, providing the most diversely 979 coloured images over regions with minimal contributions from surface dust 980 (Delamere et al 2010; McEwen et al 2010). Indeed, the colour capabilities of HiRISE 981 have also been used successfully to extend map compositional units at scales 982 below those of these spectrometers (e.g., Delamere et al 2010), and provide 983 additional insights into the spatial relationships between different spectral units 984 at a local scale. It is important to note that morphologic and stratigraphic context 985 that are often not realized in just the spectral dataset alone, especially when 
986 presented in a spectral parameter space calculated relative to the spectral 987 continuum (e.g., Pelkey et al 2007; Murchie et al 2009; Viviano-Beck et. 2014), 988 which results in an image that appears "flat" because incidence effects have 989 generally been generally divided out. In summary, the CaSSIS examples shown here demonstrate that CaSSIS will 991 provide a similar extension to spectral mapping, but with better coverage and 992 spectral fidelity then HiRISE. The improved spectral sensitivity is provided by the 993 "splitting" of the HiRISE IR band into two separate bands on CaSSIS (i.e., RED and 994 NIR; see Table 1). This added spectral capability is best exemplified by comparing 995 a simulated CaSSIS CBRC1 with a HiRISE CBRC equivalent constructed from only 3 996 bands (see Figure 14; c.f., Figure 7 and Figure 12 in Delamere et al [2010]). 997 Compared to CaSSIS, HiRISE also has a smaller footprint ( $5-6 \mathrm{~km}$ in width) and a 998 more limited colour swath (Figure 10), which is 20\% of the HiRISE nominal image 999 width ( $\sim 1$ to $1.2 \mathrm{~km}$ in width). This HiRISE colour-coverage limitation has always 1000 made it challenging to "hit" or maximise coverage of a colourful surface target, 1001 and has made it extremely challenging to plan, acquire and mosaic colour images 1002 over large sites of interest (e.g., potential landing sites). Moreover, the length of 1003 a typical full-resolution HiRISE images is generally $\sim 10 \mathrm{~km}$, but typically no more 1004 than $\sim 25 \mathrm{~km}$. CaSSIS will be able to provide full-colour images that are just over $1005 \sim 1.5 x$ the width, and up to $\sim 4 x$ the length of a typical HiRISE ( $\sim x$ the length of the 1006 image in Figure 10). As such, CaSSIS provides improved colour coverage that is $\sim 8$ 1007 times greater than HiRISE per image. Extending the mineral/phase mapping of 1008 coarser-spatial resolution orbiting spectrometers to the spatial scale of CaSSIS will 1009 be necessary for investigating the link between surface composition, and 1010 particularly subsurface composition (e.g., crater-excavated), and the origin of 1011 seasonal trace gases such as methane. These capabilities, in conjunction with the 1012 ability to acquire stereo images on a single orbit and produce DTMs from them, 1013 will facilitate a greater understanding of spectral units and their spatial 1014 relationships, and thereby greatly enhance composition and photometric 1015 investigations into key sites of interest on Mars, including those that are possible 1016 sources for transient methane in the atmosphere. 
1017 3.5.2 Change detection and Seasonal processes

1018 Aeolian Processes

1019 Aeolian bedforms (such as dunes and ripples) record the interaction 1020 between surface material and wind and provide important data about the past 1021 and current surface conditions, as well as the climate in which they formed and 1022 evolved (e.g., Greeley and Iversen 1985). High spatial resolution HiRISE 1023 orthoimages combined with DTMs have allowed for the precise quantification of 1024 dune and ripple displacement rates and for an improved understanding of the 1025 evolution of bedforms under different Martian settings and conditions (Silvestro 1026 et al 2010; Bridges et al 2011, 2013; Ayoub et al 2012; Chojnacki et al 2014, 2015, 1027 2016a; Cardinale et al 2016). However, many of these reports are only based on a 1028 few sets of observations separated by 1-2 Mars-year intervals, and are spatially 1029 limited because of the relatively small HiRISE image footprint ( $\sim-6 \mathrm{~km})$. The more 1030 than double surface coverage of a CaSSIS image will provide the means to 1031 characterise broader-scale changes in dune fields, and possibly bedform 1032 migration, provided that the CaSSIS spatial resolution and length of the time-series 1033 will be sufficient to detect the changes.

1034 Although bedform migration rates in many locations on Mars (e.g., 1035 Herschel crater, Gale crater) are frequently relatively low ( 0.3-0.4 m/year) 1036 (Silvestro et al 2013; Cardinale et al 2016), and would require long time periods 1037 for detection, several locations with faster dunes are better candidates for 1038 monitoring by CaSSIS. Nili Patera is one of such location where ripples and dunes 1039 are observed to migrate rapidly (Silvestro et al 2010; Bridges et al 2012). To test 1040 the potential for future CaSSIS dune monitoring, we generated oversampled (4.6 $1041 \mathrm{~m} /$ pixel) orthorectified CTX images of the Nili Patera dune field to assess if CaSSIS 1042 would be able to spatially resolve changes resulting from dune migration observed 1043 with HiRISE stereo-derived DTMs and orthorectified images in previous studies.

1044 Although the reported displacement at the Nili Patera dune field is on the 1045 order of, or less than, a CaSSIS or CTX pixel ( $<5 \mathrm{~m}$; see Bridges et al 2012), we 1046 detect dune displacement as changes primarily associated with lightening and 1047 darkening between the two images at the top and the bottom of the slip face, and 1048 at the trailing edge of the windward face (see Figure 15 and animated GIFs 
1050 are highlighted by normalizing the difference between the two orthorectified

1051 images, which highlight the pixels with a largest change in the relative intensity

1052 between the two images. The normalized difference image is colour-coded and

1053 then stretched to highlight these pixels, essentially highlighting changes at both

1054 the lower (darkest) and upper (brightest) ends of the histogram. These changes

1055 reflect either a change in the surface tonality/albedo (e.g., dark dune materials on

1056 the windward face moving to reveal an underlying brighter surface) or

1057 illumination. As a dune migrates, we observe Illumination changes on the slopes

1058 of slip face and near the dune crest, typically brightening along the base and

1059 darkening along the advancing crest due to a change in slope and thereby

1060 illumination. The southwest-facing slip faces of the Nili Patera dunes are

1061 illuminated due to the mid-afternoon timing of MRO observations. As the slip face

1062 advances towards the southwest, the illuminated slope also advances to the

1063 southwest. Pixels that previously covered parts of the dune with little to no slope

1064 in the before image, are now covering the base of the illuminated slope of the slip

1065 face. This is consistent with an observable brightening in the pixels near the base

1066 of the slip face in the southwest direction (i.e., magenta leading-edge in Figure 15).

1067 There is also an observable darkening of pixels at the crest of the slip face (i.e.,

1068 blue edge in Figure 15). Essentially, the pixels that are now stoss-ward from the

1069 crest and where the slope faces away from the sun in the after-image, were once

1070 on the sun-facing slopes of the slip face in the before image. We also observe a

1071 brightening of pixels at the trailing edge of the stoss-ward face (i.e., magenta

1072 training-edge in Figure 15). This is consistent with surface brightening in the pixels

1073 that occupy this area because as the dune advances the dark sands are being

1074 actively removed by saltation in this area, which reveals the underlying lighter-

1075 toned surface.

1076 [FIGURE 15]

1077 Based on the CTX orthoimages, which were acquired 7.5 Earth years ( 4

1078 Mars years) apart, the migration rate of the upwind (stoss) dunes is estimated to

1079 be $1.1 \mathrm{~m} /$ year. We note that this rate is slightly higher than the rate measured

1080 for the same dunes from HiRISE orthoimages PSP_005684_1890, and 
1081 ESP_023353_1890 ( $0.8 \mathrm{~m} /$ year), but these are only separated by just over half of 1082 the time frame of the CTX images used here. Another reason for the small 1083 discrepancy ( 1 HiRISE pixel width) could be due to the greater spacecraft-jitter 1084 issues recorded in the CTX orthoimages, and/or an overestimation of dune 1085 displacements. Alternatively, the longer-baseline estimates could be larger due to 1086 the possibility of higher annual sand fluxes in the later years spanned by the 1087 oversampled CTX measurements (2007-2014), over the HiRISE-based observations 1088 (2007-2011). Indeed, large annual variations in sand fluxes that were described 1089 elsewhere on Mars (e.g., Meridiani Planum; see Chojnacki et al 2016a) may be 1090 feasible here. In general, Nili Patera dunes have been shown to have relatively high 1091 rates with respect to other active dunes on Mars (Bridges et al 2013). Even then, 1092 only motion of the fastest dunes found in the stoss-ward section of the dune field 1093 is measurable at the oversampled $4.6 \mathrm{~m} /$ pixel resolution and a relatively long time1094 series or temporal baseline.

1095 Without ongoing studies of the Nili Patera dune field and other zones of 1096 known active aeolian activity by HiRISE and CaSSIS, previous comparisons and 1097 estimation of rates remain somewhat speculative. With the spatial resolution, 1098 colour capability, and the ability to acquire stereo pairs on a single orbit, the new 1099 CaSSIS data will be able to continue to detect changes and provide refined 1100 estimated on dune migration on Mars given the acquisition of a time-series of 1101 images over a sufficient period (i.e., several Martian years). Ripple movement 1102 detection will not likely be possible at CaSSIS resolution scales.

1103 Recurring Slope Lineae (RSL)

1104 Recurring Slope Lineae (RSL) are dark-toned linear features that initiate at 1105 bedrock outcrops and extend down steep slopes (McEwen et al 2011). Individual 1106 slopes may have hundreds of lineae, with widths up to $5 \mathrm{~m}$ and lengths as long as $11071.5 \mathrm{~km}$ (McEwen et al 2014). RSL appear and lengthen gradually or incrementally, 1108 fade when inactive, and recur each year, normally in the warmest season (McEwen 1109 et al 2011). HiRISE has also detected newly-formed slumps associated with RSL 1110 fans in at least 7 locations (Chojnacki et al 2016b), which are now part of an 1111 ongoing investigation to understand these enigmatic seasonal features. These 1112 slumps begin, like RSL, as dark-toned features that are up to $30 \mathrm{~m}$ wide, which will 
1113 be readily resolvable by CaSSIS. In some cases, RSL have been observed to leave

1114 behind bright or distinctly coloured deposits (Chojnacki et al 2016b). Seasonal RSL

1115 activity has been shown to be associated with the transient presence of hydrated

1116 salts, which strongly suggests that brines play some role in the formation (Ojha et

1117 al 2015). Recently, Dundas et al (2016) showed RSL are confined to angle-of-

1118 repose slopes or steeper $\left(>28^{\circ}\right)$, indicating they are predominantly granular flows

1119 whose activity may be triggered by or somehow associated with small amounts of

1120 water. Transient uniform darkening of fans upon which the RSL form, such as in

1121 Valles Marineris, also occur (McEwen et al 2014) and may also be due to

1122 deliquescence of small amounts of water (Heinz et al 2016) or some, as of yet,

1123 unknown process.

1124 Given the width, and colour or relatively darker-tonality of these features,

1125 in addition to the SNR, the point-spread function (PSF) and pixel-scale ( $\sim .6$

$1126 \mathrm{~m} / \mathrm{pixel}$ ) of CaSSIS, the largest observed lineae, and slopes with high densities of

1127 smaller RSL should be detectable. This is further supported by the fact that the

1128 largest RSL fans have been characterised with 6 m/pixel CTX images (Chojnacki

1129 et al 2016b). Figure 16 provides a portion of a fully-simulated CaSSIS infrared

1130 colour image covering a portion of the RSL-monitoring site in the central uplift of

1131 Horowitz crater. A close-up image reveals several dark-toned slope streaks that

1132 are indeed HiRISE-confirmed RSL (c.f., with Figure 1 in McEwen et al [2011]), which

1133 have been shown to have clear incremental seasonal growth through repeat

1134 imaging.

1135 [FIGURE 16]

1136 The time of day when RSL are most active remains unconstrained at this

1137 time. This is largely because MRO is in a Sun-synchronous orbit and can only 1138 observe in the middle afternoon - the driest time of day. However, the $74^{\circ}-$

1139 inclined polar orbit of TGO migrates through all local times 1-2 times per Mars

1140 year, affording unique opportunities to image in the early morning hours when

1141 deliquescent liquids are most stable on the surface (Gough et al 2011). CaSSIS

1142 images will be vital towards testing the "deliquescence" hypothesis for the role of

1143 brines in the origin and evolution of RSL features via the detection of early-

1144 morning wet patches and their patterns in space and time. If the RSL or their fans

1145 are transiently dark due to deliquescence (i.e., wetting), then this process is not 
1146 likely to be restricted to steep slopes; rather, it causes downhill flows (RSL) only

1147 on the steepest slopes. Therefore, a key goal for CaSSIS would be to detect

1148 transient dark patches at this earlier time of day. Unfortunately, CaSSIS can only

1149 re-image these locations in the afternoon several months before or after the

1150 morning images, so that seasonal changes will make the identification and

1151 characterization of diurnal changes difficult. Fortunately, a coordinated

1152 investigation with TGO and MRO can mitigate this issue. HiRISE and CTX can image

1153 these sites in the mid-afternoon hours within a couple of weeks of the CaSSIS

1154 early-morning images. New techniques in cross-instrument time-series change

1155 detection methods (e.g., Unsalan and Boyer, 2004) will be used to create a

1156 consistent time-series set of images that uses camera models and DTMs as inputs;

1157 these inputs are specifically used to minimize the effects of distortions, different

1158 lighting conditions and different resolutions between the images in the set, and

1159 thereby facilitate cross-instrument change detection. This technique may also be

1160 used to study changes associated with the other features described in the 1161 remaining subsections below.

1162 Active Gullies

1163 The term "gully" is used on Mars to describe a kilometre-scale erosion1164 deposition system, where material is transported downslope via a channel. They 1165 are divided into three parts: an alcove, a transport-dominated channel and a 1166 depositional apron, or fan (Malin and Edgett 2000) where the materials of the fan 1167 materials are sourced from the wall into which the alcove formed. The results from 1168 the most comprehensive global survey utilizing CTX images at $\sim 5-6 \mathrm{~m} /$ pixel 1169 indicate that well-developed gullies are found commonly on steep slopes at 1170 latitudes poleward of $30^{\circ}$ in both hemispheres, and that there are tens of 1171 thousands of individual gullies located in at least 4861 separate sites (e.g., craters, 1172 massifs, valleys, etc.) (Harrison et al 2015). Smaller gullies are also observed in 1173 equatorial regions with HiRISE images, some of which are commonly associated 1174 with RSLs (McEwen et al 2014; Auld and Dixon 2016). Gullies are found on steep 1175 slopes independent of slope type; hence, they are most commonly found on inner 1176 crater walls, but are also found on crater-rim slopes, valley walls, massifs, pits, 1177 dunes and slopes associated with both complex crater central peaks and pits (e.g., 
1178 Balme et al 2006). Between $30-40^{\circ}$ latitude in both hemispheres, gullies are almost

1179 exclusively pole-facing; poleward of $40^{\circ}$, gullies exhibit less of an orientation

1180 preference, but are generally more equator-facing (Harrison et al 2015; Conway

1181 et al 2017). When latitudinal variation is normalised as a function of the availability

1182 of steep slopes, no particular latitude appears to have a higher density of gullies

1183 than any other; they are, however, less common in areas with high concentrations

1184 of glacier-like landforms (Conway et al 2017). The orientation preference of gullies

1185 at certain latitude ranges (Harrison et al 2015; Conway et al 2017) strongly points

1186 to a link between the Martian climate and their formation (e.g., Costard et al 1187 2002).

1188 Repeat imaging by MOC, CTX, and HiRISE continues to reveal that gullies 1189 are evolving under the present-day climate regime (Malin et al 2006; Diniega et al 1190 2010; Dundas et al 2010, 2012, 2015, submitted; Harrison et al 2009; Raack et al 1191 2015). This activity is quite common, with changes observed at $14 \%$ of non-dune 1192 monitoring sites in the southern hemisphere (Dundas et al 2015). Dune gullies are 1193 reported to be even more active (e.g., Pasquon et al 2016) than gully sites that 1194 occur in bedrock and other deposits. The morphologic expression of active gullies 1195 includes the: 1) appearance of metre-to-decametre scale failure scars in the 1196 source regions; 2) evacuation/deposition of sediments on the order of metres 1197 deep from pre-existing channels; 3) transport of metre-scale boulders; 4) 1198 formation of new channels on fans; and 5) formation of new deposits. The new 1199 deposits present the largest surface area (up to hundreds of metres wide and $~ 1$ $1200 \mathrm{~km}$ in length) and range from digitate deposits with no detectable thickness at 1201 HiRISE-scale to metre-thick boulder-bearing lobes. These deposits can be light-, 1202 dark-, or neutral-toned relative to the surroundings, and are sometimes distinctive 1203 in colour. Of these HiRISE-detected changes, new deposits associated with gully 1204 activity are of a scale that are most likely observable by CaSSIS.

\section{5 [FIGURE 17]}

1206 The gullies of Gasa crater are amongst the most highly active on Mars 1207 today. The gullies in Gasa crater are notable because of their very deeply incised 1208 alcoves (de Haas et al 2015; Okubo et al 2011) and accumulation of deposits over 1209 time which has led to the formation of very large fan deposits (relative to other 
1210 gully fans on Mars) that have merged to become a bajada (Kolb et al 2010; Schon

1211 and Head 2012). At least 15 documented changes have been observed between

1212 2007-2016 (Dundas et al 2017); however, it is highly likely that all the gullies in

1213 the crater are active on timescales of a few decades or less.

$1214 \quad$ Here we present a two-image change detection time-series using two fully-

1215 simulated CaSSIS images of Gasa crater taken 2 years ( 1 Mars year) apart, one

1216 from 2009 and the other from 2011 (Figure 17). Both pre-existing light- and dark-

1217 toned gully deposits are clearly visible in our simulated CaSSIS image (Figure 17a-

1218 e), in addition to the new dark gully deposits which appeared between 2009 and

12192011 (c.f., with Figure 8 in Dundas et al 2012). Figure 17b and 17e shows new gully

1220 deposits, including one specific deposit in the northwestern corner, included and

1221 discussed in the studies by Dundas et al (2012; 2015). This deposit formed

1222 somewhere between $L_{s}=109-152^{\circ}$ of Mars Year 30 (June $24-S e p t .20,2010$ ) and

1223 resulted in substantial topographic changes at the meter scale (see animated GIF

1224 included as part of our supporting online supplemental materials and Dundas et

1225 al [2012]), including lobate deposits and channel widening. Interestingly, the new

1226 deposit in the northwestern part of Gasa closely matches the background surface

1227 brightness in a greyscale HiRISE RED image (i.e., its nearly invisible at red

1228 wavelengths), but is extremely distinctive in the colour swath of HiRISE (c.f.,

1229 Figures 8a and 8b in Dundas et al 2015). This deposit is also easily identified in our

1230 fully-simulated CaSSIS infrared colour image shown in Figure 17b. The

1231 distinctiveness in colour is attributed to NIR-band sensitivity to ferrous-bearing

1232 bedrock, originally documented by Okubo et al (2011), which can be seen exposed

1233 in the gully alcoves that have incised deeply into the carter wall of Gasa.

1234 In order to determine the process(es) responsible for new mass wasting 1235 events in gullies, it is important to understand both their seasonal timing and their 1236 relationship with seasonal volatiles (surface $\mathrm{CO}_{2}$ or water frost). Repeat-imaging 1237 with HiRISE has revealed that the majority of monitored sites are active in the 1238 winter or early spring when $\mathrm{CO}_{2}$ frost is present (Diniega et al 2010; Dundas et al 12392012,2015 ; Raack et al 2015), but the appearances of some of the low-relief light1240 toned deposits are better correlated with the presence of $\mathrm{H}_{2} \mathrm{O}$ frost (Vincendon 1241 2015), or small patches of $\mathrm{CO}_{2}$ frost (Dundas et al submitted). With its narrow field 
1242 of view, HiRISE is not able to monitor many sites frequently - especially in colour;

1243 a handful of flows can be constrained to intervals of days or weeks, but most

1244 intervals between images span months or years.

1245 The full-colour coverage over a $~ 9.4-\mathrm{km}$ swath provided by CaSSIS will be 1246 ideal for readily detecting deposits like those observed in Gasa, which are most

1247 distinctive in colour IRB images. As such, new deposits detected by CaSSIS can be

1248 later followed up with HiRISE to document the finer meter-scale details. Our

1249 simulated CaSSIS time-series set of images covering Gasa crater also demonstrates

1250 that CaSSIS will be able to clearly detect changes associated with active mass

1251 wasting features, as demonstrated by our example in which a new feature is

1252 detected in the non-gullied southwestern crater wall (c.f., Figure $17 f$ and $17 \mathrm{~g}$ ).

1253 CaSSIS will also be ideal for looking for flows burying seasonal frost (c.f., Dundas

1254 et al 2015). Furthermore, CaSSIS will be able to take images at different times of

1255 the day, which will allow us to determine whether transient morning/evening

1256 surface frosts are present at the sites of active gullies and monitor east-facing

1257 crater walls and slopes without the persistent shadowing that afternoon

1258 observations must contend with. Such observations with CaSSIS are anticipated

1259 provide vital inputs into models that will place further constraints on whether or

1260 not gullies are active via a "wet" or "dry" processes on Mars today (e.g., Pilorget

1261 and Forget 2016).

1262 New and recent impacts

1263 Over five hundred new or recent impact sites on Mars have now been

1264 imaged by MOC, CTX, and HiRISE. These recent impact sites are most easily seen

1265 due to their large dark-toned blast zones, and are most easily detected in dusty

1266 regions by the MOC wide-angle camera and/or CTX, which are then imaged and

1267 confirmed subsequently by MOC narrow-angle camera and/or HiRISE (Malin et al

1268 2006; Daubar et al 2013). HIRISE imaging shows that many of these recent impacts

1269 manifest as clusters of individual craters, which provides information on the

1270 strengths and densities of the impactor bodies that created them, impact

1271 incidence, and atmospheric thickness (e.g., Ivanov et al 2009).

1272 One of the most intriguing investigations into recent impacts on Mars

1273 revealed that they act as probes of subsurface ice in the mid- to high-latitude 
1274 regions (Byrne et al 2009; Dundas et al 2014). Based on studies of repeat imaging 1275 provided by HiRISE, the impact-exposed ice may remain bright and distinctly 1276 coloured for periods of months to years (Dundas and Byrne, 2010; Dundas et al 1277 2014); this suggests that the ice is nearly pure, and not consistent with an ice1278 cemented regolith. In Figure 18, we compare a simulated CaSSIS image with a full-

1279 resolution HiRISE IRB image of a recent 20-m diameter impact crater showing 1280 bright deposits consistent with water-ice excavated by the crater (Dundas et al 1281 2014). We can see in this comparative example that the simulated CaSSIS image 1282 reproduces most of the details of the crater that are visible to HiRISE. This includes 1283 the distribution of its dark-toned ejecta rays and, more significantly, the brighter 1284 icy deposits. This example demonstrates that CaSSIS will not only be able to 1285 support studies of current impact rates on Mars, but also provide constraints on 1286 the distribution of subsurface ice at the mid- to high-latitudes (e.g., Daubar et al 1287 2013; Dundas et al 2014). The distribution of ground ice serves as a proxy for the 1288 recent climate, integrated over the time required for ground ice to sublimate or 1289 deposit. The current extent of ice-exposing impacts acts as a lower limit on the 1290 extent of ground ice, which is more extensive than expected. This suggests an 1291 average atmospheric humidity somewhat higher than present (Byrne et al 2009; 1292 Dundas et al 2014), and/or some other factor(s) with an equivalent effect on ice 1293 stability. The larger than expected extent of ground ice in the northern 1294 hemisphere may also be inherited from previous climates rather than reflecting 1295 present day conditions making interpreting these results ambiguous. However, 1296 the southern ground ice boundary is not as sensitive to recent changes of orbital 1297 elements as that in the northern hemisphere (Chamberlain and Boynton 2007). 1298 Knowledge of the extent of the southern hemisphere ice boundary would thus 1299 help to resolve the ambiguity between the ice resulting from unexpectedly-humid 1300 present-day conditions versus being a relic of a previous climate that is 1301 unexpectedly slow to catch up to current conditions.

\section{2 [FIGURE 18]}

1303 Like CTX, CaSSIS is expected to have sufficient spatial capability for 1304 detecting new impacts in dusty regions of Mars. Although it clearly has the 1305 advantages of colour and a slightly higher spatial resolution over CTX, the CaSSIS 
1306 areal coverage will be $\sim 3$ times narrower than CTX ( $\sim 9.4$ vs. $30 \mathrm{~km}$ swath width).

1307 Additionally, the TGO data rate may be much lower than that of MRO, which will

1308 likely limit CaSSIS surface coverage. As such, CTX is likely to remain the best

1309 dataset for finding new features due to its ability to provide extensive repeat

1310 coverage and global-mosaicking capabilities, while CaSSIS and HiRISE would be

1311 able to provide follow-up investigations of any new CTX-detected recent impact

1312 candidates (Malin et al 2006; Daubar et al 2013). One exception to this is

1313 observations over low-albedo regions, where CTX images are noisy, and recent

1314 impacts do not create a large contrasted spot on the surface. This includes the

1315 majority of the southern mid- to high-latitudes, where CaSSIS may be better suited

1316 to discover new icy impacts, which are needed to determine the ice table

1317 boundary in the southern hemisphere. Because CaSSIS will be able to resolve these

1318 new impacts well, it will be an excellent tool for monitoring the darkening and

1319 reddening of their ejecta as the surface-exposed ice sublimates.

1320 Sublimation processes: Frosting/Defrosting

1321 Every winter the polar regions of Mars are covered with a seasonal polar

1322 cap composed of $\mathrm{CO}_{2}$ ice that extends down to latitudes of approximately $50^{\circ}$

1323 (Leighton and Murray 1966), with some patches of $\mathrm{CO}_{2}$ frost forming closer to the

1324 equator under favourable conditions (Schorghofer and Edgett 2005). Seasonal $\mathrm{CO}_{2}$

1325 ice deposits can reach around one metre in thickness (Smith et al 2001), which

1326 during the spring sublimates, and thereby mobilizes sediment at the Martian

1327 surface (e.g., Kieffer 2000). Despite the limited access to the poles due the TGO

1328 orbit being inclined $74^{\circ}$, CaSSIS will still have access to interesting regions with

1329 active seasonal processes shaping the terrain. With the ability to turn off-nadir,

1330 CaSSIS may be able to image near-polar targets as high as $75^{\circ}$ latitude.

1331 [FIGURE 19]

1332 A rather distinctive example of seasonal sublimation-abetted sediment 1333 mobilisation features are araniforms, or "spiders", found in the south polar 1334 regions of Mars (Malin and Edgett 2001). Figure 19 illustrates a type-locality, 1335 dubbed "Inca City", where this morphologic feature commonly occurs. These 1336 landforms are thought to be caused by jets of gas escaping through cracks from 1337 the seasonal slab of $\mathrm{CO}_{2}$ that forms on the surface in the winter months (Kieffer et 
1338 al 2006). These features are suggested to be produced by the increasing and more

1339 direct sunlight (higher sun elevation) with the progression of spring, which largely

1340 transmits through the translucent slab $\mathrm{CO}_{2}$ ice, and thereby causes the underlying

1341 surface to differentially and drastically heat-up with respect to the overlying slab

1342 ice. This differential heating between the ice and surface results in vigorous

1343 sublimation at contact between the slab ice and surface, thereby initially trapping

1344 and pressurising the sublimated gas. When a weak spot in the ice ruptures due to

1345 the mounting pressure, fractures form conduits that allow the gas to escape; with

1346 repeated activity along these conduits, branching "channels" are carved into the

1347 surface that are quasi-radial to the initiation site giving rise to their distinctive

1348 dendritic or spider-like appearance (Kieffer 2000; Piqueux et al 2003; Kieffer et al

1349 2006; Kieffer 2007). The escaping gas jets into the ambient atmosphere and

1350 deposits the surface material both locally as a dark spot and as a fan-shaped

1351 deposit with respect to the predominant direction of the wind or, in some cases,

1352 down local slopes. Dark spots and fans are clearly resolved in the simulated CaSSIS

1353 change-detection image pair, and some changes can also be discerned between

1354 the two images (Figure 19; see animated GIF included as part of our supporting

1355 online supplemental materials). Unfortunately, CaSSIS will not be able to resolve

1356 individual araniform fractures/channels that are resolved by HiRISE, so monitoring

1357 the changes of these features will specifically remain a HiRISE activity. However,

1358 CaSSIS will certainly be able to aid with systematic monitoring of their most

1359 prominent features - dark-toned spots and fans (as seen in Figure 19). New

1360 araniform detections and activity was recently aided by a HiRISE-based citizen

1361 science project. The project measured fan azimuths with the aim of using these

1362 features as a "weathervanes" for monitoring seasonal changes to local and

1363 regional wind directions (Aye et al 2016). CaSSIS image coverage and resolution

1364 would provide the means to readily extend such work since the fans are readily

1365 resolved at a decametre-scale.

1366 The observation of an active plume remains a requirement to validate the 1367 aforementioned formation-model for araniforms. Such an observation has eluded

1368 HiRISE due to the sun-synchronous orbit of MRO, an afternoon observation time,

1369 and temporal repeatability of no less than 2 hours near the poles. Models suggest 
1370 that the plumes are likely to be short-lived, and most active around noon. The

1371 capability of CaSSIS to image at various times of day, due to the orbital inclination

1372 of TGO, and the ability to capture a stereo pair mere minutes-apart, may enable

1373 observations of plumes jetting above the surface around that time of day.

1374 Although the "Inca City" site is outside the latitudes observable by CaSSIS, there

1375 are numerous araniform sites reported between $70^{\circ} \mathrm{N}$ and $75^{\circ} \mathrm{N}$ (e.g., Piqueux et

1376 al 2003). One example is HiRISE image ESP_039781_1075, which is located at

$1377 \sim 72.3^{\circ} \mathrm{S}-$ well within reach of a CaSSIS observation.

\section{8 [FIGURE 20]}

1379 A similar sublimation process for producing araniform features is thought

1380 to be responsible for seasonal dark-toned deposits that manifest as "flows" and

1381 "spots" that are visible on frost coated sand dunes in the mid- to high-latitudes of

1382 both hemispheres (Malin and Edgett 2001; Hansen et al 2013). However, some

1383 workers have argued for a role of liquid brines instead of sublimation of seasonal

1384 frost to explain these features (e.g., Kereszturi et al 2009). Figure 20 shows such a

1385 simulated CaSSIS change-detection pair location dubbed "Arrakis", which resolves

1386 multiple dark-toned spots and deposits at the base of the slopes of the dunes that

1387 appear to merge or grow between the season pairs (see animated GIF included as

1388 part of our supporting online supplemental materials). Although "Arrakis" is just

1389 beyond the reach of CaSSIS, a portion of the north polar erg observed between

$1390300^{\circ} \mathrm{E}$ and $350^{\circ} \mathrm{E}$ and below $75^{\circ}$ is accessible to CaSSIS.

1391 Another example of an active seasonal process currently under

1392 investigation are changes associated with linear dune gully features (e.g., Reiss

1393 and Jaumann 2003; Diniega et al 2013; Pasquon et al 2016). Linear dune gullies are

1394 peculiar landforms, which have only been identified on Mars, and are typified by

1395 those observed on the southwest-facing slip face of the Russell crater "mega"-

1396 dune site. Linear dune gullies are comparatively distinctive from other Martian

1397 gullies as they are characterized by an abbreviated alcove, and a long, narrow

1398 channel with little evidence of any terminal deposition. Such gullies have only

1399 been identified in the southern hemisphere of Mars and are thought to be

1400 restricted to $\sim 33$ locations at latitudes poleward of $35^{\circ}$ (Pasquon et al 2016).

1401 Pasquon et al (2016) showed that these gullies are active towards the end of the 
1402 seasonal defrosting and suggested that changes may be episodic rather than

1403 incremental. One hypothesis explaining the formation of linear dune gullies entails

1404 the initial carving or lengthening of a gully channel from blocks of ice that dislodge

1405 themselves from shadowed cold traps in alcoves, which then sublimate as they

1406 travel downward on a largely defrosted and much warmer surface (Diniega et al 1407 2013).

1408 The simulated CaSSIS infrared colour image shown in Figure 21 resolves the 1409 dune gully alcoves and channels, and distinctive colours associated with both frost 1410 and sites of sublimation (i.e., dark-toned spots and slope-streaks). The example 1411 clearly demonstrates that new channels and the lengthening of existing channels 1412 may be resolved clearly with CaSSIS. The extended CaSSIS colour coverage over

1413 HiRISE may help determine relationships between frosted surfaces and the active 1414 sites. Although highly unlikely, with the ability of CaSSIS to acquire stereo on a 1415 single orbit, it may be possible to observe the ice blocks while in motion down 1416 slope.

1417 [FIGURE 21]

1418 Studies of other seasonal changes associated with polar dunes also include 1419 the formation of new alcoves and associated debris aprons, and the erasure of 1420 older ones, which are typically undetectable with HiRISE in less than 3 years 1421 (Hansen et al 2011; Diniega et al 2017). An outstanding question of this study is: 1422 what is the driving mechanism for triggering sand avalanches that form new 1423 alcoves and fans? Is it simply aeolian degradation, or some other seasonal process 1424 driven by volatiles (Horgan and Bell 2012; Hansen et al 2015)? HiRISE already has 142510 sites that are being actively monitored every year and that are within the 1426 latitudinal imaging limits of CaSSIS. A correlation between new alcove formation 1427 and regional snowstorms is currently under investigation (Hayne et al 2016). 1428 CaSSIS images will allow us to extend our temporal and spatial colour-coverage to 1429 a larger region for analysis. CaSSIS stereo-derived DTMs will help us to determine 1430 if some dunes are over-steepened by autumnal winds, and whether they are thus 1431 susceptible to sand avalanches. New alcoves detected with HiRISE can be quite 1432 large, as much as $\sim 60$ to $100 \mathrm{~m}$ wide, which will be readily detectable by CaSSIS. 
In summary, the evolution of seasonal processes linked to defrosting and

1434 sublimation of surface ice can be monitored using CaSSIS images. Particularly 1435 useful will be the full colour-coverage, single-orbit acquisition stereo capabilities 1436 and the opportunity to image at different times of day. CaSSIS will enable 1437 improved constraints on the extent and rate of seasonal defrosting through full1438 colour coverage of the area covered by $\mathrm{CO}_{2}$ frost over the span of several images 1439 taken in a seasonal time-series. The fact that that polar dunes are so active, in 1440 addition to being comprised of low-albedo materials, has made the extraction of 1441 HiRISE or CTX stereo-derived DTMs challenging, but CaSSIS offers full-colour, at a 1442 higher SNR, and stereo images that will be taken with very little time delay, which 1443 will vastly improve our ability to produce elevation and orthorectified time-series 1444 data products required to validate the changes observed between images.

\section{3.5.3 Landing Sites and Future Exploration}

1446 One of the most important and fundamental mission objectives for Mars 1447 orbiters and their instrument payload is supporting the scientific exploration and 1448 engineering/safety of landed assets on the surface (e.g., Golombek et al 2003, 1449 2016; Grant et al 2011). This requires an assortment of datasets that generally 1450 provide information on surfaces properties that include: 1) geologic 1451 context/morphology (both context and high-resolution images); 2) 1452 topography/morphometry (elevation/slope); 3) composition (spectral and colour 1453 images); and 4) physical properties (thermal and/or radar). The uses of these 1454 datasets for this particular purpose generally entails a full-characterisation and 1455 synthesis of both the scientific merits of the site, as well as both the landing safety, 1456 traversability, and accessibility to sites of interest determined from orbit.

1457 Indeed, one of three mission objectives for CaSSIS is to: "certify and 1458 characterise safety and hazards (e.g., rocks, slopes, etc.) associated with candidate 1459 landing sites for ExoMars 2020, and other future surface missions" (Thomas et al 1460 this issue). It is important to note that this particular supportive role not only 1461 entails pre-landing characterisation, but now also includes: 1) monitoring during 1462 the Entry Decent and Landing (EDL) phase (Phoenix and Curiosity); 2) observations 1463 of landing sites immediately following EDL (Spirit, Opportunity and Phoenix); 3) 
1464 monitoring during the mission (Spirit, Opportunity, Phoenix and Curiosity); 4)

1465 observations of landing sites after the mission (Vikings 1 and 2, Pathfinder,

1466 Phoenix and Spirit); and 5) searching for and assessing what happened to "lost"

1467 missions to the surface (e.g., Schiaparelli EDM, Beagle 2 and possibly Mars 3, etc.).

1468 Such collections of landing site images have provided vast insights into pre- syn-

1469 and post-mission activities (e.g., McEwen et al 2010; Seelos et al 2014).

1470 EDL images can provide important insights into the events leading up to 1471 landing on Mars, while imaging of landing sites both before and after EDL provide 1472 the means to facilitate localization of our surface assets. The ability of HiRISE to 1473 resolve the landers/rovers themselves, in addition to rover tracks, have also been 1474 invaluable in these regards (e.g., McEwen et al 2010; Golombek et al 2010, 2011; 1475 Geissler et al 2011; Arvidson et al 2015). High-resolution orbital images provide 1476 the best resource for determining both local- and regional-scale geologic context 1477 and for matching observations with rover/lander acquired panoramic image 1478 mosaics. This is not as easily accomplished with spectral and other types of 1479 datasets that are coarser resolution (e.g., MOLA and THEMIS, etc.), but high1480 resolution orbital images may act as a "bridge" to match these coarser resolution 1481 datasets to that of the local-scale of the lander/rover. The augmented geologic 1482 context provided by the synthesis of the in situ investigations of by surface 1483 missions with orbital datasets, including decameter-scale geologic mapping of 1484 landing sites, have facilitated the long-term planning of active roving missions, and 1485 maximised the science return of both landed and roving missions alike (e.g., 1486 Golombek et al 2003; McEwen et al 2010; Grant et al 2011). Meter- to decameter1487 scale elevation data derived from stereo images are critical towards assessing 1488 potential landing site hazards, and the traversability of a landing site and its 1489 surrounding regions for long-term planning and science investigations of complex 1490 features, such as the ejecta and rim-wall region of impact craters (e.g., Golombek 1491 et al 2003; McEwen et al 2010).

1492 In this section, we provide simulated CaSSIS images as examples of past, 1493 current and future candidate landing sites. Although surface roughness and slope 1494 are two of the most important engineering parameters to assess during the 
1495 landing site proposal and selection stages (e.g., Golombek et al 2003; Kirk et al 1496 2003, 2008; Beyer and Kirk 2012; Fergason et al 2016), it is beyond the scope of 1497 this particular study to perform a full hazard and safety assessment of our 1498 simulated CaSSIS landing site images. Our goal is to simply assess the ability of 1499 anticipated CaSSIS images to resolve and characterise various aspects of a landing 1500 site, as well as provide site monitoring support for an active surface mission.

$1501 \quad$ Before presenting and discussing our simulated CaSSIS images covering 1502 various landing sites, it is worth noting two examples of post-EDL imaging by CTX, 1503 which is a reasonable proxy for the spatial capabilities of CaSSIS as these two 1504 imagers have similar pixel-scales. The post-EDL imaging of the Phoenix lander is 1505 our first example. Figure S7 shows a close-up of a low emission angle $\left(\sim 0.5^{\circ}\right) \sim 6$ $1506 \mathrm{~m} /$ pixel CTX image taken on July 20th in 2008 of the Phoenix landing site, which 1507 resolves three dark spots and one bright one. Of the three dark spots, one is 1508 relatively larger than the other two, with the other two being more or less similar 1509 in size. One of these similar-sized dark spots (slightly lighter than the other) is 1510 associated with a nearby cluster of bright pixels, which are consistent with the 1511 backshell impact site and the parachute, respectively. The other similar-sized 1512 (slightly darker) dark spot is consistent with the impact site of the heatshield. The 1513 largest dark spot is attributed to the removal of unconsolidated, light-toned and 1514 fine-grained surface materials from the firing of the retrorockets of Phoenix lander 1515 during landing. A single darker pixel can be observed in the central region of the 1516 largest dark spot and is suggested to possibly represent the Phoenix lander. The 1517 lander when fully deployed is approximately 5.52 meters in diameter, so it is 1518 conceivable. If CTX had colour capabilities, like HiRISE and CaSSIS does, it may have 1519 been possible to determine if the dark pixel is indeed consistent with the lander, 1520 as solar panels are distinctive in the infrared colour image. Indeed, a rescaled 1521 HiRISE IRB image, using a pixel aggregate resampling algorithm to rescale to 4.6 $1522 \mathrm{~m} /$ pixel, shows a single blue pixel in the central region of the largest dark spot, 1523 which is consistent with subpixel mixing of the relatively dust-free solar panels of 1524 the Phoenix lander early on in the mission (Figure S7).

1525 Our second CTX-based example is based on the post-EDL image of the 1526 ExoMars Schiaparelli EDL Demonstrator Module (EDM). Due to the fact that the 
1527 exact location of the EDM was initially unknown, and the large 30-km swath width

1528 of a CTX image, CTX was the first to document and observe surface changes

1529 associated with the components of the Schiaparelli EDM (see MRO-views of the

1530 Schiaparelli EDM Landing Site). Despite the high emission angle $\left(\sim 25^{\circ}\right)$ and a $\sim 6$

$1531 \mathrm{~m} /$ pixel spatial resolution of the initial CTX image (see CTX image 1532 J08_47975_1779_02S006W), the location of surface changes associated with the

1533 four crucial components of the Schiaparelli EDM were resolved. This includes the 1534 parachute, the impact sites of the module, and both the rear (attached to the 1535 parachute) and front heatshields. The information provided by CTX, and the 1536 subsequent follow-up with HiRISE colour, in conjunction with spacecraft 1537 telemetry, were critical to the investigation of the EDL events that led to the 1538 demise of the Schiaparelli EDM.

1539 These two above CTX examples indicate that CaSSIS will be able to resolve 1540 some of the aspects of future EDL events on Mars. Of course, the higher SNR and 1541 colour capabilities will provide additional benefits in this regard.

\section{Landing Sites of Past and Current Missions}

1543 In this section, we describe three fully-simulated CaSSIS images of landing 1544 sites from past and ongoing missions.

1545 Figure 22 shows portions of a fully-simulated CaSSIS infrared colour image 1546 constructed from a coordinated CRISM cube and CTX image of the Mars 1547 Exploration Rover (MER) "Spirit" landing site in Gusev crater, taken on the $15^{\text {th }}$ of 1548 February 2010. This was just one month before Spirit fell silent near the location 1549 known as "Troy" (red star in Figure 22) just to the west of the 90-m wide feature 1550 known as "Home Plate". This is the location where the rover got entrenched in 1551 unconsolidated deposits almost one year prior to when the CRISM-CTX-HiRISE 1552 image set was acquired. The Spirit rover landing site (orange star in Figure 22), 1553 rover, and rover tracks are not resolvable in the simulated CaSSIS image; however, 1554 numerous dust storm events, and dust devil activity since Spirit arrived the 1555 Columbia Hills in 2006, likely erased any distinctive surface changes associated 1556 with the EDL and traverse of Spirit prior to its long-term science investigation at 1557 the "Home Plate". 
Figure S8 shows a comparison of the coverage HiRISE IRB images and the

1560 surface coverage provided by a single fully-simulated CaSSIS infrared colour image, 1561 which is approximately $10 \mathrm{~km}$ at its narrowest (i.e., close to the nominal $9.4 \mathrm{~km}$ 1562 swath width of CaSSIS). This comparison example demonstrates the challenges of 1563 obtaining HiRISE IRB coverage with reasonably similar observation angles, and the 1564 relative ease the CaSSIS full-colour swath-width will provide colour context. This 1565 is particularly relevant for this example, as the Columbia Hills and surrounding 1566 plans, which are an active site of change with respect to dust devil activity; as such, 1567 mosaics produced from images over active sites, and where sufficient time has 1568 elapsed between acquisitions, will suffer from multiple mismatches. The full1569 colour coverage of CaSSIS will provide more than $8 x$ the colour coverage of a 1570 HiRISE colour image ( 1-1.2 km wide), providing colour coverage and context for 1571 landing sites as well as other key sites of interest.

Figure 23 shows two fully-simulated CaSSIS images of the Curiosity MSL 1573 rover landing site. One of the landing site shortly after EDL, and including coverage 1574 of a portion of the long-term science target for MSL - Aeolis Mons (i.e., "Mount 1575 Sharp"). The fully-simulated CaSSIS image of Aeolis Mons, or Mount Sharp, 1576 provides a beautiful infrared colour context image that may prove useful for MSL 1577 long-term planning; indeed, the exploration of the Gale central mound is just 1578 beginning as of writing this paper. While, the post-EDL image of the Curiosity MSL 1579 rover taken in January of 2013, 5 months after landing, shows similarities to both 1580 the post-EDL CTX images of Phoenix lander (see Figure S7) and the Schiaparelli 1581 EDM images. Our fully-simulated CaSSIS image shows that the landing site, and 1582 the descent stage ("sky crane") multiple-impact site, can all be resolved with some 1583 detail. Unlike the previous Spirit rover example, rover tracks are indeed present 1584 and resolvable with full-resolution HiRISE images taken at the time of the MRO 1585 coordinated image set observations used for our simulation. As such, this 1586 particular fully-simulated CaSSIS image provides a better test to observe if the 1587 surface contrasts produced by rover tracks are sufficient to effect subpixel mixing 1588 to where they might be resolved. Unfortunately, and even when considering the 1589 larger size of MSL with respect to the MERs, neither the rover nor the rover tracks 
1590 are resolvable in the simulated image. This is even the case when using the HiRISE

1591 RED mosaic image resampled to the CaSSIS pixel-scale instead of CTX. While it is

1592 not likely that CaSSIS will resolve landed assets on the Martian surface or rover

1593 tracks from its nominal 400-km orbit, it might be possible to resolve these features

1594 from a lower orbit. Resampling the HiRISE RED mosaic image to $2 \mathrm{~m} /$ pixel shows

1595 that the rover tracks are discernible, which suggests that resolving such features

1596 might be possible in the event that the TGO orbit is adjusted to include a periapsis

1597 of $\sim 200 \mathrm{~km}$.

1598 [FIGURE 23]

1599 Landing Sites for Future Missions: ExoMars 2020 and Mars 2020

1600 In 2013, the ESA ExoMars 2020 landing site call was released to the

1601 scientific community, which included two overarching mission objectives: 1) to

1602 search for signs of past and present life on Mars; and 2) to investigate the

1603 water/geochemical environment as a function of depth in the shallow subsurface.

1604 To satisfy these requirement eight landing sites were suggested (seven located

1605 around Chryse Planitia, and one located on Isidis Planitia), all characterised by

1606 evidence in support of potentially long exposures to aqueous environments (e.g.,

1607 Erkeling et al 2013; Vago et al 2017; Pajola et al 2016a). At the time of writing this

1608 paper, only two of the three finalists from early 2017 (Bridges et al 2017) are

1609 currently under consideration. One is the Oxia Planum site, an extensive clay-

1610 bearing flat $300 \mathrm{~km}$ across, located at the just south of the margin of the crustal

1611 dichotomy and Chryse Planitia (Carter et al 2016; Quantin et al 2016; Pajola et al

1612 2017b), and the other is situated amongst the well-exposed clay-bearing terrain

1613 of Mawrth Vallis (Gross et al 2016).

1614 Figure 24 shows a simulated CaSSIS synthetic "true" colour image and

1615 simulated CaSSIS CBRC1 image covering the southeastern portion of the Oxia

1616 Planum landing ellipse. In HiRISE images the "capping unit" and smectite clay-

1617 bearing unit, described by previous workers (Carter et al 2016; Quantin et al 2016),

1618 seems to have different VNIR colours than the majority of the surface. The

1619 "capping unit" manifests as a generally spectrally "bland", darker-toned crater

1620 retaining unit (also see Figure S9). Although the smectite clay-bearing unit does 
1621 not appear to be distinctive in our fully-simulated CaSSIS synthetic "true" colour

1622 (or infrared colour - not shown), the unit can be readily mapped as an orange unit

1623 in the simulated CBRC1 image (Figure 24). This is due to the ferric absorptions

1624 associated with the Fe-bearing smectites observed in CRISM data (Carter et al

1625 2016; Quantin et al 2016). The CBRC1 orange unit is consistent with a subtle, light-

1626 toned beige unit (orange arrows) in the RGB image, which underlies the distinctive

1627 "capping unit". This and other examples herein clearly demonstrate that CaSSIS-

1628 based CBRC images are particularly useful for expanding the spectral mapping

1629 from CRISM (and other spectral datasets). We note that there are similar

1630 relationships between different colour units in Mawrth Vallis (Gross et al 2016) as

1631 the one described and shown here with the Oxia Planum example. That would be

1632 readily mapped out in CaSSIS images.

\section{3 [FIGURE 24]}

1634 Following the definition of the scientific threshold criteria for the NASA 1635 Mars 2020 landing site call in 2013 (Mustard, Beaty and Bass 2013), multiple

1636 landing sites have been proposed (e.g., Rice et al 2013; Edwards and EhImann

1637 2015; Pajola et al 2016a, 2016b). The scientific selection criteria state that a

1638 candidate landing site must provide: 1) a geologic setting and history that can be

1639 characterised from orbit and in situ; 2) preservation of an ancient habitable

1640 environment; 3) access to samples with high biosignature preservation potential;

1641 and 4) returned samples of abundance, diversity, and quality that will be used to

1642 address questions regarding astrobiology; and planetary evolution.

1643 As of writing this paper, eight landing sites for Mars 2020 (see Golombek

1644 et al 2016b) have been narrowed down to 3 finalists by the scientific community.

1645 The currently favoured Mars 2020 landing site is Jezero crater (Figure S10), which

1646 is located in the Syrtis Major quadrangle. The crater is thought to have once been

1647 flooded with water, and currently contains a clay-bearing fan-shaped deposit that

1648 is consistent with a lacustrine delta (see Goudge et al 2015). This site is followed

1649 by: the northeast portion of Syrtis Major (a.k.a., "NE Syrtis"), and the Columbia

1650 Hills within the Gusev crater basin (Figure 22). All eight sights going into the $3^{\text {rd }}$

1651 Mars 2020 landing site workshop, held in February of 2017, showed substantial

1652 scientific merit (Golombek et al 2016b). It is conceivable that one of the sites that 
1653 did not make the final cut, sites such as Eberswalde crater (Figure S11), Mawrth

1654 Vallis (Figure S12), "Nili Fossae Trough" (Figures 9d, 25 and S13), Southwestern

1655 Melas Basin, and Holden crater, may still be considered over the next year or so

1656 based on any redeeming engineering safety merits.

1657 By combining the spatial and colour details with elevation data provided

1658 by CaSSIS, and synthesizing them with spectrometer data such as CRISM, we would

1659 not only greatly enhance the strategic and long-term planning of a rover mission,

1660 but improve on our ability for the rover to sample and cache the diverse

1661 compositions that are dispersed throughout a given landing site. As an example,

1662 we present a portion of a fully-simulated CaSSIS infrared colour image of the

1663 scoured deposits of the Hargraves ejecta blanket (Figure 25), which covers the

1664 eastern portion of the proposed Mars 2020 rover "Nili Fossae Trough" landing

1665 ellipse. The Hargraves crater ejecta, observed and described in brief by McEwen

1666 et al (2010), offers access to one of the best-exposed ejecta blankets on Mars. The

1667 ejecta exhibits a sharp juxtaposition of diverse "mega"-brecciated clasts and

1668 matrix materials comprised of the diverse target lithologies offered by the Nili

1669 Fossae region. The target materials of this region largely include unaltered, ferrous

1670 iron-bearing materials (pyroxene-bearing clasts and possible impact melt-bearing

1671 materials), and altered ferric iron-bearing materials consistent with $\mathrm{Fe} / \mathrm{Mg}$

1672 smectites, but also includes other alteration phases such as serpentine and

1673 carbonates. Both infrared colour and CBRC1 images in Figure 25 show sharp

1674 contacts between unaltered (blues in the CBRC1) and altered (yellows/oranges in

1675 the CBRC1) surface materials representing chemical redox gradients. Notably,

1676 these sharp redox boundaries have been shown to be important sites on Earth for

1677 sustaining metabolic processes for chemotrophic life (e.g., De Marais et al 2003).

1678 CaSSIS is well-designed to map out these Fe-redox boundaries in detail and will

1679 thus provide support for planning in situ investigations, and sampling, of the best-

1680 candidate materials that may preserve biosignatures of chemophilic lifeforms.

1681 [FIGURE 25] 
1683 et al this issue; Cremonese et al 2017) for assessing the engineering and safety

1684 constraints of landing sites, CaSSIS will provide some improvements over HiRISE

1685 towards the scientific characterisation of landing sites. This includes improved

1686 NIR-sensitivity, higher coverage in full-colour, and the means for rapid stereo

1687 completion and DTM production. The larger footprint and full-colour coverage will

1688 facilitate the extension of spectral and colour mapping needed to plan and explore

1689 within a landing ellipse or beyond (i.e., "go to" sites of interest). In the past, it has

1690 been extremely difficult to acquire complete coverage of large landing ellipses

1691 with imagers that have small image footprints on the surface (MOC-NAC and

1692 HiRISE); thus, some landing site characterization studies have had to rely on

1693 staggered surface statistics (e.g., the Insight lander; see Golombek et al 2016).

1694 CaSSIS has $~ 1.5 x$ the width and up to $4 x$ the length of a typical HiRISE image; as

1695 such, CaSSIS will improve our ability to cover and mosaic a landing ellipse and

1696 various surrounding sites of interest. The $4.6 \mathrm{~m} / \mathrm{pixel}$ full-colour and CBRC mosaics

1697 possible with CaSSIS will enable decameter-scale mapping, both morphologically

1698 and spectrally/thermophysically via the extension of coarser resolution datasets.

1699 These synthesized map products will provide invaluable geologic context. The

1700 synthesis and context provided by these products with with the in situ results from

1701 the suite of instruments included as part of the payload of the rover will augment

1702 long-term planning goals on an active mission, and maximise our understanding

1703 of the geologic history and potential habitability of the site being explored.

1704 In the event of a future EDL failure, and assuming future landed hardware

1705 size and mass are similar to recent missions, a CaSSIS image immediately following

1706 the event would be able to answer several questions such as: 1) Did the parachute

1707 open? and 2) Did the heat shield and backshell separate? A "no" to the first

1708 question would likely result in an image of a single disturbed surface spot or small

1709 crater. If "yes", we would expect to see a few bright pixels representing the

1710 parachute on the surface. If "yes" for question two, we would see additional

1711 disturbed surface spots representing the impact sites for these components. 


\section{Summary \& Conclusions}

1713 This study uses the instrument response function for the Colour and Stereo

1714 Surface Imaging System (CaSSIS) imager on the ExoMars Trace Gas Orbiter (TGO)

1715 to assess the sensitivity of the anticipated dataset to common phases on Mars

1716 identified by past orbiting and in situ platforms. This study also provides a

1717 methodology to produce a robusts simulation, both spectrally and spatially, of

1718 CaSSIS image cubes that are constructed from previously acquired and

1719 coordinated Mars Reconnaissance Orbiter (MRO) observations.

$1720 \quad$ Using these simulated CaSSIS products, our analysis focuses on assessing

1721 the spatial and spectral performance and capabilities of CaSSIS, and provides

1722 insight into the best suggestions for higher-level derived colour data products to

1723 produce when real CaSSIS data are obtained. For example, prescribed sets of

1724 band-ratio colour composites are shown to enhance studies that are keen on

1725 identifying the spatial distribution of ferrous $\left(\mathrm{Fe}^{2+}\right)$ and ferric $\left(\mathrm{Fe}^{3+}\right)$ surface

1726 materials, as well as rapidly differentiating between ice/frost and atmospheric

1727 phenomena from other surface materials for seasonal, atmospheric and

1728 climatological studies (e.g., haze, fog, clouds, dust devils, avalanche clouds, etc.).

1729 These simulations also demonstrate that CaSSIS images will continue to provide

1730 insight into spatial and temporal changes that occur due to various active surface

1731 phenomena (e.g., seasonal deposition/sublimation of ices, gullies, RSL, dust

1732 avalanche streaks, dust devil tracks, landed/roving surface missions, etc.).

1733 Despite the lower spatial resolution of CaSSIS when compared to HiRISE,

1734 this work demonstrates that CaSSIS will not only supplement HiRISE-scale

1735 investigations of various geological and seasonal phenomena, but enhance them

1736 by providing: 1) additional geologic context with its wider and longer full-colour

1737 coverage; 2 ) increased sensitivity to iron-bearing materials from two IR bands (RED

1738 and NIR) with the ability to produce colour band ratio composite images to

1739 facilitate mapping the spatial distribution and stratigraphic relations of

1740 compositionally diverse surface materials; 3) greater flexibility in monitoring

1741 seasonal and recent recurrent changes due to variations in local time of image

1742 acquisition enabled by the TGO orbit; and 4) near-simultaneous acquisition of

1743 stereo images (along-track stereo), which will ensure the stereo pairs are always 
1744 taken under similar atmospheric and illumination conditions, and perhaps even

1745 capture rapidly evolving processes "in action" (e.g., dust devils, $\mathrm{CO}_{2}$ jets, 1746 avalanches, etc).

1747 Our simulated CaSSIS images demonstrate the detection of subtle surface 1748 changes that were either ambiguous or not sampled by the HiRISE colour swath or 1749 footprint (e.g., Gasa crater active gullies, and additional dune movements in Nili 1750 Patera). This result demonstrates the power of the improved spatial colour1751 coverage of CaSSIS over HiRISE, as well as demonstrating that CaSSIS has sufficient 1752 resolving power to detect most surface changes currently being studied with 1753 HiRISE. However, we note that surface mission landers/rovers and rover tracks 1754 were not resolved in our simulated images. This clearly demonstrates the need for 1755 a full-colour sub-meter pixel-scale imager on a future orbiter (e.g., on the Next 1756 Mars Orbiter - NeMO 2022) to enable the monitoring and tracking of future 1757 robotic, and possibly manned, missions to Mars.

1758 Lastly, spectral pan-sharpening is commonly used for terrestrial datasets 1759 to mitigate data management and volume issues, or when there is a lack of 1760 coverage by other high-resolution colour imaging platforms. Indeed, the 1761 robustness of our methodology, and the resulting fully-simulated CaSSIS image 1762 cube product substantiates them as a stand-alone product, not just for the 1763 planning for the acquisition of real CaSSIS images and calibration efforts, but as a 1764 supplemental colour product for comparison and co-analysis with other Mars 1765 datasets. Fully-simulated CaSSIS image cubes may also be used to extend a time 1766 series set of images used for the monitoring of seasonal targets and change 1767 detection sites to the pre-TGO era (i.e., fully-simulated CaSSIS cubes produced 1768 from coordinated MRO datasets spanning 2006 to present). These simulated 1769 products will provide the means to detect changes that occur over longer time1770 scales on Mars (e.g., dune migration). Finally, we recommend that colour/spectral 1771 pan-sharpening of higher-resolution panchromatic grayscale images from CTX and 1772 HiRISE, beyond this simulation, would also be of great benefit to ongoing studies 1773 of the Martian surface. We note that the methodology detailed herein can be 1774 readily modified towards this specific goal. These products would be immediately 1775 useful where colour-coverage may be vital, and where HiRISE colour coverage is 
1776 specifically lacking or difficult to achieve. Indeed, the use of actual CaSSIS colour

1777 images to pan-sharpen available HiRISE RED mosaic grayscale images is not only

1778 desirable, but highly recommended for high-level investigations of the Martian

1779 surface, such as landing site characterisation, selection and exploration.

1780 The results of this study indicate that the TGO mission objectives, as well

1781 as the instrument-specific mission objectives, will be achievable with CaSSIS.

\section{Acknowledgements}

1783 The authors wish to thank the spacecraft and instrument engineering teams for the successful

1784 completion of the instrument. CaSSIS is a project of the University of Bern and funded through

1785 the Swiss Space Office via ESA's PRODEX programme. The instrument hardware development

1786 was also supported by the Italian Space Agency (ASI) (ASI-INAF agreement no.I/018/12/0),

1787 INAF/Astronomical Observatory of Padova, and the Space Research Center (CBK) in Warsaw.

1788 Support from SGF (Budapest), the University of Arizona (Lunar and Planetary Lab.) and NASA are

1789 also gratefully acknowledged.

1790 The lead author wishes to personally acknowledge funding and support through the Planetary

1791 [ExoMars] Co-Investigator programme from the Canadian Space Agency (CSA) (14EXPUWO-002)

1792 and a Canadian NSERC Discovery Grant programme (RGPIN/04215-2014). A special thanks to the

1793 science and operations teams of the CRISM, CTX and HiRISE instruments, from which the

1794 spectacular coordinated datasets/images, which were required to simulate CaSSIS, would not be

1795 possible.

1796 We would also like to acknowledge DTM technician Allison McGraw at University of Arizona for 1797 her contribution towards generating the HiRISE and CTX stereo-derived DTMs used in this study. 
1800 Adams J. B., Lunar and martian surfaces: petrologic significance of absorption bands in the near-infrared. Science 159, 1453-1455 (1968)

1803

1804

1805

1806

1807

1808

1809

1810

1811

1812

1813

1814

1815

1816

1817

1818

1819

1820

1821

1822

1823

1824

1825

1826

1827

1828

1829

1830

1831

1832

1833

1834

1835

1836

1837

1838

1839

1840

1841

1842

1843

1844

Adams J. B., McCord T. B., Mars: Interpretation of spectral reflectivity of light and dark regions. J. Geophys. Res. 74(20), 4851-4856 (1969)

Adams J.B., Visible and near-infrared diffuse reflectance spectra of pyroxenes as applied to remote sensing of solid objects in the solar system. Journal of Geophysical Research 79(32), pp.4829-4836 (1974)

Aharonson O., Zuber M.T., Smith D.E., Neumann, G.A., Feldman, W.C., Prettyman T.H., Depth, distribution, and density of $\mathrm{CO} 2$ deposition on Mars. Journal of Geophysical Research: Planets 109, E5 (2004)

Appéré T. et al., Winter and spring evolution of northern seasonal deposits on Mars from OMEGA/Mars Express. J. Geophys. Res. 116, E05001 (2011). http:// dx.doi.org/10.1029/2010JE003762

Arvidson, R.E., Bell, J.F., Catalano, J.G., Clark, B.C., Fox, V.K., Gellert, R., Grotzinger, J.P., Guinness, E.A., Herkenhoff, K.E., Knoll, A.H. and Lapotre, M.G.A., 2015. Mars Reconnaissance Orbiter and Opportunity observations of the Burns formation: crater hopping at Meridiani Planum. Journal of Geophysical Research: Planets, 120 (3), 429-451.

Auld, K. S., Dixon, J. C. (2016). A classification of martian gullies from HiRISE imagery. Planetary and Space Science, 131, 88-101.

Aye, K. M., Schwamb, M. E., Portyankina, G., \& Hansen, C. J. (2016). Analysis Pipeline and Results from the PlanetFour Citizen Science Project. 47th Lunar and Planetary Science Conference abst. 3056.

Ayoub, F., Avouac, J.P., Newman, C.E., Richardson, M.I., Lucas, A., Leprince, S. and Bridges, N.T., 2014. Threshold for sand mobility on Mars calibrated from seasonal variations of sand flux. Nature communications, 5.

Bailen, M.S., Sucharski, R.M., Akins, S.W., Hare, T.M. and Gaddis, L.R., 2013, March. Using the PDS Planetary Image Locator Tool (PILOT) to Identify and Download Spacecraft Data for Research. In Lunar and Planetary Science Conference (Vol. 44, p. 2246).

Balme, M., Mangold, N., Baratoux, D., Costard, F., Gosselin, M., Masson, P., Pinet, P., Neukum, G., 2006. Orientation and distribution of recent gullies in the southern hemisphere of Mars: Observations from High Resolution Stereo Camera/Mars Express (HRSC/MEX) and Mars Orbiter Camera/Mars Global Surveyor (MOC/MGS) data. J. Geophys. Res. Planets 111, doi:10.1029/2005JE002607.

Bandfield, J.L., Hamilton, V.E. and Christensen, P.R., 2000. A global view of Martian surface compositions from MGS-TES. Science, 287(5458), pp.1626-1630.

Bell, J. F., Wolff, M. J., James, P. B., Clancy, R. T., Lee, S. W., \& Martin, L. J. (1997). Mars surface mineralogy from Hubble Space Telescope imaging during 1994-1995: Observations, calibration, and initial results. Journal of Geophysical Research: Planets, 102(E4), 9109-9123.

Becerra, P., Byrne, S. and Brown, A.J., 2015. Transient bright "halos" on the South Polar Residual Cap of Mars: Implications for mass-balance. Icarus, 251, pp.211-225. 
1845

1846

1847

1848

1849

1850

1851

1852

1853

1854

1855

1856

1857

1858

1859

1860

1861

1862

1863

1864

1865

1866

1867

1868

1869

1870

1871

1872

1873

1874

1875

1876

1877

1878

1879

1880

1881

1882

1883

1884

1885

1886

1887

1888

1889

1890

1891

Beyer, R. A., \& Kirk, R. L. (2012). Meter-scale slopes of candidate MSL landing sites from point photoclinometry. Space science reviews, 170(1-4), 775-791.

Bishop, J.L., Parente, M., Weitz, C.M., Noe Dobrea, E.Z., Roach, L.H., Murchie, S.L., McGuire, P.C., McKeown, N.K., Rossi, C.M., Brown, A.J. and Calvin, W.M., 2009. Mineralogy of Juventae Chasma: Sulfates in the light-toned mounds, mafic minerals in the bedrock, and hydrated silica and hydroxylated ferric sulfate on the plateau. Journal of Geophysical Research: Planets, 114(E2).

Bridges, J. C., Loizeau, D., Sefton-Nash, E., Vago, J., Williams, R. M. E., Balme, M., ... \& WG, E. L. S. S. (2017). Selection and Characterisation of the ExoMars 2020 Rover Landing Sites. Lunar and Planetary Science 48, 1964, 2378.

Bridges, N.T., Ayoub, F., Avouac, J.P., Leprince, S., Lucas, A. and Mattson, S., 2012a. Earth-like sand fluxes on Mars. Nature, 485(7398), pp.339-342.

Bridges, N.T., Bourke, M.C., Geissler, P.E., Banks, M.E., Colon, C., Diniega, S., Golombek, M.P., Hansen, C.J., Mattson, S., McEwen, A.S. and Mellon, M.T., 2012b. Planet-wide sand motion on Mars. Geology, 40(1), pp.31-34.I

Bridges, N.T., Geissler, P., Silvestro, S. and Banks, M., 2013. Bedform migration on Mars: Current results and future plans. Aeolian Research, 9, pp.133-151.

Burns, R.G., 1970. Mineralogical applications of crystal field theory, Cambridge University Press, New York.

Burns, R. G. (1993). In Remote Geochemical Analysis: Elemental and Mineralogical Composition, edited by C.M. Pieters, and P.A.J. Englert, Cambridge University Press, 4, 3-29.

Byrne, S., Dundas, C.M., Kennedy, M.R., Mellon, M.T., McEwen, A.S., Cull, S.C., Daubar, I.J., Shean, D.E., Seelos, K.D., Murchie, S.L. and Cantor, B.A., 2009. Distribution of mid-latitude ground ice on Mars from new impact craters. Science, 325(5948), pp.1674-1676.

Calvin, W.M., Roach, L.H., Seelos, F.P., Seelos, K.D., Green, R.O., Murchie, S.L. and Mustard, J.F., 2009. Compact Reconnaissance Imaging Spectrometer for Mars observations of northern Martian latitudes in summer. Journal of Geophysical Research: Planets, 114(E2).

Cardinale, M., Silvestro, S., Vaz, D.A., Michaels, T., Bourke, M.C., Komatsu, G. and Marinangeli, L., 2016. Present-day aeolian activity in Herschel Crater, Mars. Icarus, 265, pp.139-148.

Carter, J., Poulet, F., Bibring, J.P., Mangold, N. and Murchie, S., 2013. Hydrous minerals on Mars as seen by the CRISM and OMEGA imaging spectrometers: Updated global view. Journal of Geophysical Research: Planets, 118(4), pp.831-858.

Carter, J., Quantin, C., Thollot, P., Loizeau, D., Ody, A. and Lozach, L., 2016, March. Oxia Planum: A Clay-Laden Landing Site Proposed for the ExoMars Rover Mission: Aqueous Mineralogy and Alteration Scenarios. In Lunar and Planetary Science Conference (Vol. 47, p. 2064).

Caudill, C.M., Tornabene, L.L., McEwen, A.S., Byrne, S., Ojha, L. and Mattson, S., 2012. Layered MegaBlocks in the central uplifts of impact craters. Icarus, 221(2), pp.710-720.

Chamberlain, M.A. and Boynton, W.V., 2007. Response of Martian ground ice to orbit-induced climate change. Journal of Geophysical Research: Planets, 112(E6). 
1892

1893

1894

1895

1896

1897

1898

1899

1900

1901

1902

1903

1904

1905

1906

1907

1908

1909

1910

1911

1912

1913

1914

1915

1916

1917

1918

1919

1920

1921

1922

1923

1924

1925

1926

1927

1928

1929

1930

1931

1932

1933

1934

1935

1936

1937

1938

Chojnacki, M., Burr, D.M. and Moersch, J.E., 2014. Valles Marineris dune fields as compared with other martian populations: Diversity of dune compositions, morphologies, and thermophysical properties. Icarus, 230, pp.96-142.

Chojnacki, M., Johnson, J.R., Moersch, J.E., Fenton, L.K., Michaels, T.I. and Bell, J.F., 2015. Persistent aeolian activity at Endeavour crater, Meridiani Planum, Mars; new observations from orbit and the surface. Icarus, 251, pp.275290.

Chojnacki, M., Urso, A., Fenton, L. K., \& Michaels, T. I. (2016a). Aeolian dune sediment flux heterogeneity in Meridiani Planum, Mars. Aeolian Research.

Chojnacki, M., A. McEwen, C. Dundas, L. Ojha, A. Urso, and S. Sutton (2016b), Geologic context of recurring slope lineae in Melas and Coprates Chasmata, Mars, J. Geophys. Res. Planets, 121, 1204-1231, doi:10.1002/2015JE004991.

Christensen, P.R., Bandfield, J.L., Smith, M.D., Hamilton, V.E. and Clark, R.N., 2000a. Identification of a basaltic component on the Martian surface from Thermal Emission Spectrometer data. Journal of Geophysical Research, 105(E4), pp.9609-9621.

Christensen, P.R., Bandfield, J.L., Clark, R.N., Edgett, K.S., Hamilton, V.E., Hoefen, T., Kieffer, H.H., Kuzmin, R.O., Lane, M.D., Malin, M.C. and Morris, R.V., 2000b. Detection of crystalline hematite mineralization on Mars by the Thermal Emission Spectrometer: Evidence for near-surface water. Journal of Geophysical Research: Planets, 105(E4), pp.9623-9642.

Christensen, P.R., Bandfield, J.L., Bell III, J.F., Gorelick, N., Hamilton, V.E., Ivanov, A., Jakosky, B.M., Kieffer, H.H., Lane, M.D., Malin, M.C. and McConnochie, T., 2003. Morphology and composition of the surface of Mars: Mars Odyssey THEMIS results. Science, 300(5628), pp.2056-2061.

Christensen, P., Wyatt, M., Glotch, T., Rogers, A., Anwar, S., Arvidson, R., . . Wolff, M. (2004). Mineralogy at Meridiani Planum from the Mini-TES Experiment on the Opportunity Rover. Science, 306(5702), 1733-1739. Retrieved from http://www.jstor.org/stable/3839768

Christensen, P.R., Engle, E., Anwar, S., Dickenshied, S., Noss, D., Gorelick, N. and Weiss-Malik, M., 2009, December. JMARS-A Planetary GIS. In AGU Fall Meeting Abstracts (Vol. 1, p. 06).

Clark, R.N., Swayze, G.A., Gallagher, A.J., King, T.V. and Calvin, W.M., 1993. The US Geological Survey, Digital Spectral Library: Version 1 (0.2 to 3.0 um) (No. 93-592). Geological Survey (US).

Clark, R.N., Swayze, G.A., Wise, R., Livo, K.E., Hoefen, T., Kokaly, R.F. and Sutley, S.J., 2007. USGS digital spectral library splib06a. US Geological Survey, Digital Data Series, 231.

Conway, S.J., Harrison, T.N., Soare, R.J., Britton, A., Steele, L., 2017. Reanalysis of global data on orientation and slope of gullies on Mars. Geol. Soc. Lond. Spec. Publ. in review.

Costard, F., Forget, F., Mangold, N., Peulvast, J.P., 2002. Formation of recent Martian debris flows by melting of near-surface ground ice at high obliquity. Science 295, 110-113. doi:10.1126/science.1066698

Cremonese, G., Simioni, E., Re, C., Mudric, T., Lucchetti, A., Massironi, M., ... \& Thomas, N. (2017). First Mars Surface Stereo Reconstruction with the 
CaSSIS Stereo Camera. Lunar and Planetary Science Conference 48, 1964, 1464.

D'Aoust, Bianca, "Morphological, Structural and Spectral Mapping of the Central Uplifts of the Alga and Verlaine Craters, Mars" (2015). Electronic Thesis and Dissertation Repository. 3441. http://ir.lib.uwo.ca/etd/3441

Daubar, I.J., McEwen, A.S., Byrne, S., Kennedy, M.R. and Ivanov, B., 2013. The current martian cratering rate. Icarus, 225(1), pp.506-516.

de Haas, T., Conway, S.J., Krautblatter, M., 2015. Recent (Late Amazonian) enhanced backweathering rates on Mars: paracratering evidence from gully-alcoves? J. Geophys. Res. Planets 120, 2169-2189. doi:10.1002/2015JE004915

Delamere, W.A., Tornabene, L.L., McEwen, A.S., Becker, K., Bergstrom, J.W., Bridges, N.T., Eliason, E.M., Gallagher, D., Herkenhoff, K.E., Keszthelyi, L. and Mattson, S., 2010. Color imaging of Mars by the High Resolution Imaging Science Experiment (HiRISE). Icarus, 205(1), pp.38-52.

Des Marais, D. J., Nuth III, J. A., Allamandola, L. J., Boss, A. P., Farmer, J. D., Hoehler, T. M., ... \& Spormann, A. M. (2003). The NASA astrobiology roadmap. Astrobiology, 8(4), 715-730.

Ding, N., Bray, V.J., McEwen, A.S., Mattson, S.S., Okubo, C.H., Chojnacki, M. and Tornabene, L.L., 2015. The central uplift of Ritchey crater, Mars. Icarus, 252, pp.255-270.

Diniega, S., Byrne, S., Bridges, N.T., Dundas, C.M., McEwen, A.S., 2010. Seasonality of present-day Martian dune-gully activity. Geology 38, 1047-1050. doi:10.1130/G31287.1

Diniega, S., Hansen, C.J., McElwaine, J.N., Hugenholtz, C.H., Dundas, C.M., McEwen, A.S. and Bourke, M.C., 2013. A new dry hypothesis for the formation of martian linear gullies. Icarus, 225(1), pp.526-537.

Diniega, S., C.J. Hansen, A. Allen, N. Grigsby, Z. Li, T. Perez, M. Chojnacki, Duneslope activity due to frost and wind, throughout the north polar erg, Mars. Geol. Soc. London Spec. Pub., (2017) in press, doi:

Dundas, C.M., McEwen, A.S., Diniega, S., Byrne, S., Martinez-Alonso, S., 2010. New and recent gully activity on Mars as seen by HiRISE. Geophys. Res. Lett. 37, doi:10.1029/2009gl041351. doi:10.1029/2009gl041351

Dundas, C.M., Diniega, S., Hansen, C.J., Byrne, S., McEwen, A.S., 2012. Seasonal activity and morphological changes in martian gullies. Icarus 220, 124-143. doi:10.1016/j.icarus.2012.04.005

Dundas, C.M., Byrne, S., McEwen, A.S., Mellon, M.T., Kennedy, M.R., Daubar, I.J. and Saper, L., 2014. HiRISE observations of new impact craters exposing Martian ground ice. Journal of Geophysical Research: Planets, 119(1), pp.109-127.

Dundas, C.M., Diniega, S., McEwen, A.S., 2015. Long-Term Monitoring of Martian Gully Formation and Evolution with MRO/HiRISE. Icarus 251, 244-263. doi:10.1016/j.icarus.2014.05.013

Dundas, C.M., McEwen, A.S. and Milazzo, M.P., 2016, March. How wet is recent Mars? Insights from Gullies and RSL. In Lunar and Planetary Science Conference (Vol. 47, p. 2327). 
1985

1986

1987

1988

1989

1990

1991

1992

1993

1994

1995

1996

1997

1998

1999

2000

2001

2002

2003

2004

2005

2006

2007

2008

2009

2010

2011

2012

2013

2014

2015

2016

2017

2018

2019

2020

2021

2022

2023

2024

2025

2026

2027

2028

2029

2030

2031

2032

Dundas, C. M., McEwen, A. S., Diniega, S., Hansen, C. J., Byrne, S., McElwaine, J. N. The formation of gullies on Mars today. Geol. Soc. London Spec. Pub., in press, doi:10.1144/SP467.5.

Edwards, C.S., Christensen, P.R. and Hamilton, V.E., 2008. Evidence for extensive olivine-rich basalt bedrock outcrops in Ganges and Eos chasmas, Mars. Journal of Geophysical Research: Planets, 113(E11).

Edwards, C.S. and Ehlmann, B.L., 2015, December. Carbon Sequestration on Mars: Constraints from the Nili Fossae Carbonate Plains. In AGU Fall Meeting Abstracts.

Ehlmann, B.L., Mustard, J.F., Murchie, S.L., Poulet, F., Bishop, J.L., Brown, A.J., Calvin, W.M., Clark, R.N., Des Marais, D.J., Milliken, R.E. and Roach, L.H., 2008. Orbital identification of carbonate-bearing rocks on Mars. Science, 322(5909), pp.1828-1832.

Ehlmann, B.L., Mustard, J.F., Swayze, G.A., Clark, R.N., Bishop, J.L., Poulet, F., Des Marais, D.J., Roach, L.H., Milliken, R.E., Wray, J.J. and Barnouin-Jha, O., 2009. Identification of hydrated silicate minerals on Mars using MROCRISM: Geologic context near Nili Fossae and implications for aqueous alteration. Journal of Geophysical Research: Planets, 114(E2).

Ehlmann, B.L., Mustard, J.F. and Murchie, S.L., 2010. Geologic setting of serpentine deposits on Mars. Geophysical research letters, 37(6).

EhImann, B.L., Mustard, J.F., Murchie, S.L., Bibring, J.P., Meunier, A., Fraeman, A.A. and Langevin, Y., 2011. Subsurface water and clay mineral formation during the early history of Mars. Nature, 479(7371), pp.53-60.

Ehlmann, B.L. and Edwards, C.S., 2014. Mineralogy of the Martian surface. Annual Review of Earth and Planetary Sciences, 42, pp.291-315.

Erkeling, G., Reiss, D., Hiesinger, H., Poulet, F., Carter, J., Ivanova, M.A., Hauber, E. and Jaumann, R., 2013. Two New Candidate Landing Sites for the European 2018 ExoMars Mission Near Libya Montes Alluvial Fans, In Lunar and Planetary Science Conference, (Vol. 44, p. 2378)

Fergason, R.L., Kirk, R.L., Cushing, G., Galuszka, D.M., Golombek, M.P., Hare, T.M., Howington-Kraus, E., Kipp, D.M. and Redding, B.L., 2016. Analysis of Local Slopes at the InSight Landing Site on Mars. Space Science Reviews, pp.125.

Fernando, J., Schmidt, F., \& Douté, S. (2016). Martian surface microtexture from orbital CRISM multi-angular observations: A new perspective for the characterization of the geological processes. Planetary and Space Science, 128, 30-51.

Fernando, J., Douté, S., McEwen, A., Byrne, S., Thomas, N. (2017). Mars atmospheric dust contamination of surface albedo and color measurements. In Lunar and Planetary Science Conference, vol. 48, p. 1635. 2014.

Fonti, S., and Marzo, G. A. (2010). Mapping the methane on Mars. Astronomy \& Astrophysics, 512, A51.

Formisano, V., Atreya, S., Encrenaz, T., Ignatiev, N., \& Giuranna, M. (2004). Detection of methane in the atmosphere of Mars. Science, 306(5702), 1758-1761.

Gendrin, A., Mangold, N., Bibring, J.P., Langevin, Y., Gondet, B., Poulet, F., Bonello, G., Quantin, C., Mustard, J., Arvidson, R. and LeMouélic, S., 2005. Sulfates 
in Martian layered terrains: the OMEGA/Mars Express view. Science, 307(5715), pp.1587-1591.

Gellert, R., et al. (2006), Alpha particle X-ray spectrometer (APXS): Results from Gusev crater and calibration report, J. Geophys. Res., 111, E02S05, doi:10.1029/2005JE002555.

Geissler, P.E., Sullivan, R., Golombek, M., Johnson, J.R., Herkenhoff, K., Bridges, N., Vaughan, A., Maki, J., Parker, T. and Bell, J., 2010. Gone with the wind: Eolian erasure of the Mars Rover tracks. Journal of Geophysical Research: Planets, 115(E7).

Glotch, T.D., Bandfield, J.L., Tornabene, L.L., Jensen, H.B. and Seelos, F.P., 2010. Distribution and formation of chlorides and phyllosilicates in Terra Sirenum, Mars. Geophysical Research Letters, 37(16).

Golombek, M.P., Grant, J.A., Parker, T.J., Kass, D.M., Crisp, J.A., Squyres, S.W., Haldemann, A.F.C., Adler, M., Lee, W.J., Bridges, N.T. and Arvidson, R.E., 2003. Selection of the Mars Exploration Rover landing sites. Journal of Geophysical Research: Planets, 108(E12).

Golombek, M., Robinson, K., McEwen, A., Bridges, N., Ivanov, B., Tornabene, L. and Sullivan, R., 2010. Constraints on ripple migration at Meridiani Planum from Opportunity and HiRISE observations of fresh craters. Journal of Geophysical Research: Planets, 115(E7).

Golombek, M., Grant, J., Kipp, D., Vasavada, A., Kirk, R., Fergason, R., Bellutta, P., Calef, F., Larsen, K., Katayama, Y. and Huertas, A., 2012. Selection of the Mars Science Laboratory landing site. Space science reviews, 170(1-4), pp.641-737.

Golombek, M., Kipp, D., Warner, N., Daubar, I.J., Fergason, R., Kirk, R.L., Beyer, R., Huertas, A., Piqueux, S., Putzig, N.E. and Campbell, B.A., 2016a. Selection of the InSight landing site. Space Science Reviews, pp.1-91.

Golombek, M.P., Grant, J.A., Farley, K.A., Williford, K., Chen, A., Otero, R.E. and Ashley, J.W., 2016b, March. Downselection of landing sites proposed for the Mars 2020 Rover Mission. In Lunar and Planetary Science Conference (Vol. 47, p. 2324).

Gough, R.V., Chevrier, V.F. and Tolbert, M.A., 2016. Formation of liquid water at low temperatures via the deliquescence of calcium chloride: Implications for Antarctica and Mars. Planetary and Space Science, 131, pp.79-87.

Grant, J.A., Golombek, M.P., Grotzinger, J.P., Wilson, S.A., Watkins, M.M., Vasavada, A.R., Griffes, J.L. and Parker, T.J., 2011. The science process for selecting the landing site for the 2011 Mars Science Laboratory. Planetary and Space Science, 59(11), pp.1114-1127.

Greeley, R., and Iversen, J.D., 1985, Wind as a geological process on Earth, Mars, Venus and Titan: Cambridge Planetary Science Series 4: Cambridge, Cambridge University Press, $333 \mathrm{p}$.

Hamilton, V.E. and Christensen, P.R., 2005. Evidence for extensive, olivine-rich bedrock on Mars. Geology, 33(6), pp.433-436.

Hansen, C.J., Bourke, M., Bridges, N.T., Byrne, S., Colon, C., Diniega, S., Dundas, C., Herkenhoff, K., McEwen, A., Mellon, M. and Portyankina, G., 2011. Seasonal erosion and restoration of Mars' northern polar dunes. Science, 331(6017), pp.575-578. 
Hansen, C.J., Byrne, S., Portyankina, G., Bourke, M., Dundas, C., McEwen, A., Mellon, M., Pommerol, A. and Thomas, N., 2013. Observations of the northern seasonal polar cap on Mars: I. Spring sublimation activity and processes. Icarus, 225(2), pp.881-897.

Hansen, C.J., Diniega, S., Bridges, N., Byrne, S., Dundas, C., McEwen, A. and Portyankina, G., 2015. Agents of change on Mars' northern dunes: $\mathrm{CO}_{2}$ ice and wind. Icarus, 251, pp.264-274.

Hare, T.M., Akins, S.W., Sucharski, R.M., Bailen, M.S. and Anderson, J.A., 2013, March. Map projection web service for PDS images. In Lunar and Planetary Science Conference (Vol. 44, p. 2068).

Harrison, T. N. , Malin, and K. S. Edgett (2009), Present-day Gully Activity Observed by the Mars Reconnaissance Orbiter (MRO) Context Camera (CTX), Bulletin of the American Astronomical Society 41, 3, 113, abstract 57.03.

Harrison, T.N., Osinski, G.R., Tornabene, L.L., Jones, E., 2015. Global Documentation of Gullies with the Mars Reconnaissance Orbiter Context Camera and Implications for Their Formation. Icarus 252, 236-254. doi:10.1016/j.icarus.2015.01.022

Hayne, P.O., Hansen, C.J., Byrne, S., Kass, D.M., Kleinböhl, A., Piqueux, S., McCleese, D.J., Diniega, S. and Portyankina, G., 2016. SNOWFALL VARIABILITY AND SURFACE CHANGES IN THE POLAR REGIONS OF MARS. LPI Contributions, 1926.

Heinz, J., Schulze-Makuch, D., \& Kounaves, S. P. (2016). Deliquescence-induced wetting and RSL-like darkening of a Mars analogue soil containing various perchlorate and chloride salts. Geophysical Research Letters, 43(10), 48804884.

Hoefen, T. M., Clark, R. N., Bandfield, J. L., Smith, M. D., Pearl, J. C., \& Christensen, P. R. (2003). Discovery of olivine in the Nili Fossae region of Mars. Science, 302(5645), 627-630.

Holm, N.G., Oze, C., Mousis, O., Waite, J.H. and Guilbert-Lepoutre, A., 2015. Serpentinization and the formation of $\mathrm{H} 2$ and $\mathrm{CH} 4$ on celestial bodies (planets, moons, comets). Astrobiology, 15(7), pp.587-600.

Hopkins, R.T., Tornabene, L.L. and Osinski, G.R., 2017. The central uplift of Elorza Crater: Insights into its geology and possible relationships to the Valles Marineris and Tharsis regions. Icarus, 284, pp.284-304.

Horgan, B.H. and Bell, J.F., 2012. Seasonally active slipface avalanches in the north polar sand sea of Mars: Evidence for a wind-related origin. Geophysical Research Letters, 39(9).

Hunt, G. R., and Salisbury, J. W., 1970, Visible and near-infrared spectra of minerals and rocks-I. Silicate minerals, Modern Geology, v. 1, p. 283-300.

Hunt, G.R., 1977. Spectral signatures of particulate minerals in the visible and near infrared. Geophysics, 42(3), pp.501-513.

Hunt, G.R. and Ashley, R.P., 1979. Spectra of altered rocks in the visible and near infrared. Economic Geology, 74(7), pp.1613-1629.

Ivanov, B.A., Melosh, H.J., McEwen, A.S., HiRISE Team, 2009. Small impact crater clusters in high resolution HiRISE images - II. Lunar Planet. Sci. 40. Abstract 1410. 
James, P. B., Clancy, R. T., Lee, S. W., Martin, L. J., Singer, R. B., Smith, E., ... \& Zurek, 1991 observations. Icarus, 109(1), 79-101.

Kieffer, H.H., 2000, August. Annual punctuated CO2 slab-ice and jets on Mars. In Second International Conference on Mars Polar Science and Exploration (Vol. 1, p. 93).

Kieffer, H.H., Christensen, P.R. and Titus, T.N., 2006. CO2 jets formed by sublimation beneath translucent slab ice in Mars' seasonal south polar ice cap. Nature, 442(7104), pp.793-796.

Kieffer, H.H., 2007. Cold jets in the Martian polar caps. Journal of Geophysical Research: Planets, 112(E8).

Kirk, R.L., Howington-Kraus, E., Redding, B., Galuszka, D., Hare, T.M., Archinal, B.A., Soderblom, L.A. and Barrett, J.M., 2003. High-resolution topomapping of candidate MER landing sites with Mars Orbiter Camera narrow-angle images. Journal of Geophysical Research: Planets, 108(E12).

Kirk, R. L., Howington-Kraus, E., Rosiek, M. R., Anderson, J. A., Archinal, B. A., Becker, K. J., ... \& Holmberg, I. M. (2008). Ultrahigh resolution topographic mapping of Mars with MRO HiRISE stereo images: Meter-scale slopes of candidate Phoenix landing sites. Journal of Geophysical Research: Planets, 113(E3).

Klingelhöfer, G., Morris, R., Bernhardt, B., Schröder, C., Rodionov, D., De Souza, P., ... Arvidson, R. (2004). Jarosite and Hematite at Meridiani Planum from Opportunity's Mössbauer Spectrometer. Science, 306(5702), 1740-1745. Retrieved from http://www.jstor.org/stable/3839769

Koeppen, W.C. and Hamilton, V.E., 2008. Global distribution, composition, and abundance of olivine on the surface of Mars from thermal infrared data. Journal of Geophysical Research: Planets, 113(E5).

Kolb, K.J., McEwen, A.S., Pelletier, J.D., 2010. Investigating gully flow emplacement mechanisms using apex slopes. Icarus 208, 132-142.

Kounaves, S. P., Hecht, M. H., Kapit, J., Quinn, R. C., Catling, D. C., Clark, B. C., ... \& Shusterman, J. (2010). Soluble sulfate in the martian soil at the Phoenix landing site. Geophysical Research Letters, 37(9).

Kereszturi, A., Möhlmann, D., Berczi, S., Ganti, T., Horvath, A., Kuti, A., Sik, A. and Szathmary, E., 2010. Indications of brine related local seepage phenomena on the northern hemisphere of Mars. Icarus, 207(1), pp.149-164.

Laben, C.A. and Brower, B.V., Eastman Kodak Company, 2000. Process for enhancing the spatial resolution of multispectral imagery using pansharpening. U.S. Patent 6,011,875.

Langevin, Y., Poulet, F., Bibring, J.P. and Gondet, B., 2005. Sulfates in the north polar region of Mars detected by OMEGA/Mars Express. Science, 307(5715), pp.1584-1586.

Leighton, R.B. and Murray, B.C., 1966. Behavior of carbon dioxide and other volatiles on Mars. Science, 153(3732), pp.136-144.

Malin, M.C., Edgett, K.S., 2000. Evidence for recent groundwater seepage and surface runoff on Mars. Science 288, 2330-2335. doi:10.1126/science.288.5475.2330 
Malin, M.C. and Edgett, K.S., 2001. Mars global surveyor Mars orbiter camera: interplanetary cruise through primary mission. Journal of Geophysical Research: Planets, 106(E10), pp.23429-23570.

Malin, M.C., Edgett, K.S., Posiolova, L.V., McColley, S.M., Dobrea, E.Z.N., 2006. Present-day impact cratering rate and contemporary gully activity on Mars. Science 314, 1573-1577.

Mangold, N., Poulet, F., Mustard, J.F., Bibring, J.P., Gondet, B., Langevin, Y., Ansan, V., Masson, P., Fassett, C., Head, J.W. and Hoffmann, H., 2007. Mineralogy of the Nili Fossae region with OMEGA/Mars Express data: 2. Aqueous alteration of the crust. Journal of Geophysical Research: Planets, 112(E8).

Martin, P.D., Pinet, P.C., Bacon, R., Rousset, A. and Bellagh, F., 1996. Martian surface mineralogy from 0.8 to $1.05 \mu \mathrm{m}$ TIGER spectro-imagery measurements in Terra Sirenum and Tharsis Montes formation. Planetary and space science, 44(8), pp.859-888.

Maurer, T., 2013. How to pan-sharpen images using the Gram-Schmidt pansharpen method-a recipe. International Archives of the Photogrammetry, Remote Sensing and Spatial Information Sciences, 1, p.W1.

McCord, T. B. and Westphal, J. A., 1971. Mars: narrow-band photometry from 0.3 to 2.5 microns of surface regions during the 1969 apparition. Astrophys. J. 168, 141-153.

McCord, T. B., Huguenin, R. L., Mink, D. and Pieters, C. M., 1977. Spectral reflectance of martian areas during the 1973 opposition: Photoelectric filter photometry 0.33-I. $10 \mathrm{pm}$. Icarus 31, 25-39.

McCord, T. B., Clark, R. N. and Huguenin, R. L., 1978. Mars: Near-infrared spectral reflectance and compositional implication. J. Geophys. Res., 83(BII), 54335441.

McLennan, S.M., Anderson, R.B., Bell, J.F., Bridges, J.C., Calef, F., Campbell, J.L., Clark, B.C., Clegg, S., Conrad, P., Cousin, A. and Des Marais, D.J., 2014. Elemental geochemistry of sedimentary rocks at Yellowknife Bay, Gale crater, Mars. Science, 343(6169), p.1244734.

McSween, H.Y., 2002. The rocks of Mars, from far and near. Meteoritics \& Planetary Science, 37(1), pp.7-25.

McEwen, A. S., \& Soderblom, L. A. (1984). High-resolution color images of lo. LUNAR AND PLANETARY SCIENCE XV, P. 529. Abstract.

McEwen, A.S., Eliason, E.M., Bergstrom, J.W., Bridges, N.T., Hansen, C.J., Delamere, W.A., Grant, J.A., Gulick, V.C., Herkenhoff, K.E., Keszthelyi, L. and Kirk, R.L., 2007. Mars reconnaissance orbiter's high resolution imaging science experiment (HiRISE). Journal of Geophysical Research: Planets, 112(E5).

McEwen, A.S., Banks, M.E., Baugh, N., Becker, K., Boyd, A., Bergstrom, J.W., Beyer, R.A., Bortolini, E., Bridges, N.T., Byrne, S. and Castalia, B., 2010. The high resolution imaging science experiment (HiRISE) during MRO's primary science phase (PSP). Icarus, 205(1), pp.2-37.

McEwen, A.S., Ojha, L., Dundas, C.M., Mattson, S.S., Byrne, S., Wray, J.J., Cull, S.C., Murchie, S.L., Thomas, N. and Gulick, V.C., 2011. Seasonal flows on warm Martian slopes. Science, 333(6043), pp.740-743.

McEwen, A.S., Dundas, C.M., Mattson, S.S., Toigo, A.D., Ojha, L., Wray, J.J., Chojnacki, M., Byrne, S., Murchie, S.L. and Thomas, N., 2014. Recurring 
slope lineae in equatorial regions of Mars. Nature geoscience, $7(1)$, pp.5358.

McLennan, S. M., Anderson, R. B., Bell, J. F., Bridges, J. C., Calef, F., Campbell, J. L., ... \& Des Marais, D. J. (2014). Elemental geochemistry of sedimentary rocks at Yellowknife Bay, Gale crater, Mars. Science, 343(6169), doi:10.1126/science.1244734.

Morris, R. V., et al. (2006a), Mössbauer mineralogy of rock, soil, and dust at Gusev crater, Mars: Spirit's journey through weakly altered olivine basalt on the plains and pervasively altered basalt in the Columbia Hills, J. Geophys. Res., 111, E02S13, doi:10.1029/2005JE002584.

Morris, R. V., et al. (2006b), Mössbauer mineralogy of rock, soil, and dust at Meridiani Planum, Mars: Opportunity's journey across sulfate-rich outcrop, basaltic sand and dust, and hematite lag deposits, J. Geophys. Res., 111, E12S15, doi:10.1029/2006JE002791.

Mumma, M. J., Villanueva, G. L., Novak, R. E., Hewagama, T., Bonev, B. P., DiSanti, M. A., ... \& Smith, M. D. (2009). Strong release of methane on Mars in northern summer 2003. Science, 323(5917), 1041-1045.

Murchie, S., Arvidson, R., Bedini, P., Beisser, K., Bibring, J.P., Bishop, J., Boldt, J., Cavender, P., Choo, T., Clancy, R.T. and Darlington, E.H., 2007. Compact reconnaissance imaging spectrometer for Mars (CRISM) on Mars reconnaissance orbiter (MRO). Journal of Geophysical Research: Planets, 112(E5).

Mustard, J.F., Poulet, F., Head, J.W., Mangold, N., Bibring, J.P., Pelkey, S.M., Fassett, C.I., Langevin, Y. and Neukum, G., 2007. Mineralogy of the Nili Fossae region with OMEGA/Mars Express data: 1 . Ancient impact melt in the Isidis Basin and implications for the transition from the Noachian to Hesperian. Journal of Geophysical Research: Planets, 112(E8).

Mustard, J.F., Murchie, S.L., Pelkey, S.M., Ehlmann, B.L., Milliken, R.E., Grant, J.A., Bibring, J.P., Poulet, F., Bishop, J., Dobrea, E.N. and Roach, L., 2008. Hydrated silicate minerals on Mars observed by the Mars Reconnaissance Orbiter CRISM instrument. Nature, 454(7202), pp.305-309.

Mustard, J.F., Ehlmann, B.L., Murchie, S.L., Poulet, F., Mangold, N., Head, J.W., Bibring, J.P. and Roach, L.H., 2009. Composition, morphology, and stratigraphy of Noachian crust around the Isidis basin. Journal of Geophysical Research: Planets, 114(E2).

Ockert-Bell, M. E., J. F. Bell, J. B. Pollack, C. P. McKay, and F. Forget (1997), Absorption and scattering properties of the Martian dust in the solar wavelengths, J. Geophys. Res., 102, 9039-9050, doi:10.1029/96JE03991.

Ojha, L., Wilhelm, M.B., Murchie, S.L., McEwen, A.S., Wray, J.J., Hanley, J., Massé, M. and Chojnacki, M., 2015. Spectral evidence for hydrated salts in recurring slope lineae on Mars. Nature Geoscience, 8(11), pp.829-832.

Okubo, C.H., Tornabene, L.L., Lanza, N.L., 2011. Constraints on mechanisms for the growth of gully alcoves in Gasa crater, Mars, from two-dimensional stability assessments of rock slopes. Icarus 211, 207-221.

Osinski, G.R., Tornabene, L.L. and Grieve, R.A., 2011. Impact ejecta emplacement on terrestrial planets. Earth and Planetary Science Letters, 310(3), pp.167181. 
Osterloo, M.M., Hamilton, V.E., Bandfield, J.L., Glotch, T.D., Baldridge, A.M., Christensen, P.R., Tornabene, L.L. and Anderson, F.S., 2008. Chloridebearing materials in the southern highlands of Mars. Science, 319(5870), pp.1651-1654.

Osterloo, M.M., Anderson, F.S., Hamilton, V.E. and Hynek, B.M., 2010. Geologic context of proposed chloride-bearing materials on Mars. Journal of Geophysical Research: Planets, 115(E10).

Oze, C. and Sharma, M., 2005. Have olivine, will gas: Serpentinization and the abiogenic production of methane on Mars. Geophysical Research Letters, 32(10).

Pajola, M., Rossato, S., Baratti, E., Mangili, C., Mancarella, F., McBride, K., \& Coradini, M. (2016a). The Simud-Tiu Valles hydrologic system: A multidisciplinary study of a possible site for future Mars on-site exploration. Icarus, 268, 355-381.

Pajola, M., Rossato, S., Carter, J., Baratti, E., Pozzobon, R., Erculiani, M. S., ... \& McBride, K. (2016b). Eridania Basin: An ancient paleolake floor as the next landing site for the Mars 2020 rover. Icarus, 275, 163-182.

Pajola, M., Simioni, E., Lucchetti, A., Re, C., Cremonese, G., Thomas, N., Pommerol, A., Tornabene, L. \& the CaSSIS Team (2017a). Refining the boundary between the Phobos Blue/Red spectral units with the ExoMars-CaSSIS imagery. European Planetary Science Congress 2017, Vol. 11, EPSC201768-1.

Pajola, M., Rossato, S., Baratti, E., Pozzobon, R., Quantin, C., Carter, J., \& Thollot, P. (2017b, in press). Boulder abundances and size-frequency distributions on Oxia Planum-Mars: Scientific implications for the 2020 ESA ExoMars rover. Icarus.

Pasquon, K., Gargani, J., Massé, M., Conway, S.J., 2016. Present-day formation and seasonal evolution of linear dune gullies on Mars. Icarus 274, 195-210. doi:10.1016/j.icarus.2016.03.024

Pelkey, S.M., Mustard, J.F., Murchie, S., Clancy, R.T., Wolff, M., Smith, M., Milliken, R., Bibring, J.P., Gendrin, A., Poulet, F. and Langevin, Y., 2007. CRISM multispectral summary products: Parameterizing mineral diversity on Mars from reflectance. Journal of Geophysical Research: Planets, 112(E8).

Pilorget, C., \& Forget, F. (2016). Formation of gullies on Mars by debris flows triggered by $\mathrm{CO} 2$ sublimation. Nature Geoscience, 9(1), 65-69.

Piqueux, S., Byrne, S., \& Richardson, M. I. (2003). Sublimation of Mars's southern seasonal $\mathrm{CO} 2$ ice cap and the formation of spiders. Journal of Geophysical Research: Planets, 108(E8).

Planetary Data System (PDS), Geosciences Node, Spectral Library: http://speclib.rsl.wustl.edu/, accessed 2017

Quantin, C., Flahaut, J., Clenet, H., Allemand, P. and Thomas, P., 2012. Composition and structures of the subsurface in the vicinity of Valles Marineris as revealed by central uplifts of impact craters. Icarus, 221(1), pp.436-452.

Raack, J., Reiss, D., Appéré, T., Vincendon, M., Ruesch, O., Hiesinger, H., 2015. Present-Day Seasonal Gully Activity in a South Polar Pit (Sisyphi Cavi) on Mars. Icarus 251, 226-243. doi:j.icarus.2014.03.040 
2313

2314

2315

2316

2317

2318

2319

2320

2321

2322

2323

2324

2325

2326

2327

2328

2329

2330

2331

2332

2333

2334

2335

2336

2337

2338

2339

2340

2341

2342

2343

2344

2345

2346

2347

2348

2349

2350

2351

2352

2353

2354

2355

2356

2357

2358

2359

Reiss, D. and Jaumann, R., 2003. Recent debris flows on Mars: Seasonal observations of the Russell Crater dune field. Geophysical Research Letters, 30(6).

Rieder, R., Gellert, R., Anderson, R., Brückner, J., Clark, B., Dreibus, G., . . . Zipfel, J. (2004). Chemistry of Rocks and Soils at Meridiani Planum from the Alpha Particle X-ray Spectrometer. Science, 306(5702), 1746-1749. Retrieved from http://www.jstor.org/stable/3839770

Roach, L.H., Mustard, J.F., Murchie, S.L., Bibring, J.P., Forget, F., Lewis, K.W., Aharonson, O., Vincendon, M. and Bishop, J.L., 2009. Testing evidence of recent hydration state change in sulfates on Mars. Journal of Geophysical Research: Planets, 114(E2).

Roloff, V., et al. On-Ground Performance and Calibration of the ExoMars Trace Gas Orbiter CaSSIS Imager, submitted to SSR, November 2016

Schon, S.C., Head, J.W., 2012. Gasa impact crater, Mars: Very young gullies formed from impact into latitude-dependent mantle and debris-covered glacier deposits? Icarus 218, 459-477. doi:10.1016/j.icarus.2012.01.002

Schorghofer, N. and Edgett, K.S., 2006. Seasonal surface frost at low latitudes on Mars. Icarus, 180(2), pp.321-334.

Seelos, F. P., S. L. Murchie, D. C. Humm, O. S. Barnouin, F. Morgan, H. W. Taylor, C. Hash, and CRISM Team (2011a), CRISM Data Processing and Analysis Products Update - Calibration, Correction, and Visualization, in Lunar and Planetary Institute Science Conference Abstracts, vol. 42, p. 1438.

Seelos, F. P., S. L. Murchie, A. McGovern, M. P. Milazzo, and K. E. Herkenhoff (2011b), CRISM/HiRISE Correlative Spectroscopy, in AGU Fall Meeting Abstracts, vol. 23, p. 1714.

Seelos, F. P., M. F. Morgan, H. W. Taylor, S. L. Murchie, D. C. Humm, K. D. Seelos, O. S. Barnouin, C. E. Viviano, and CRISM Team (2012), CRISM Map Projected Targeted Reduced Data Records (MTRDRs) - High Level Analysis and Visualization Data Products, in Planetary Data: A Workshop for Users and Software Developers, vol. Open-File Report 2014-1056, pp. 159-162, USGS, Flagstaff, AZ.

Seelos, F. P., C. E. Viviano-Beck, M. F. Morgan, G. Romeo, J. J. Aiello, and S. L. Murchie (2016), CRISM Hyperspectral Targeted Observation PDS Product Sets - TERs and MTRDRs, vol. 47, p. 1783.

Seelos, K. D., F. P. Seelos, C. E. Viviano-Beck, S. L. Murchie, R. E. Arvidson, B. L. Ehlmann, and A. A. Fraeman (2014), Mineralogy of the MSL Curiosity landing site in Gale crater as observed by MRO/CRISM, Geophys. Res. Lett., 41, 4880-4887, doi:10.1002/2014GL060310.

Silvestro, S., Vaz, D.A., Fenton, L.K. and Geissler, P.E., 2011. Active aeolian processes on Mars: A regional study in Arabia and Meridiani Terrae. Geophysical Research Letters, 38(20).

Silvestro, S., Vaz, D.A., Ewing, R.C., Rossi, A.P., Fenton, L.K., Michaels, T.I., Flahaut, J. and Geissler, P.E., 2013. Pervasive aeolian activity along rover Curiosity's traverse in Gale Crater, Mars. Geology, 41(4), pp.483-486.

Skok, J.R., Mustard, J.F., Tornabene, L.L., Pan, C., Rogers, D. and Murchie, S.L., 2012. A spectroscopic analysis of Martian crater central peaks: Formation of the ancient crust. Journal of Geophysical Research: Planets, 117(E11). 
Smith, D.E., Zuber, M.T. and Neumann, G.A., 2001. Seasonal variations of snow depth on Mars. Science, 294(5549), pp.2141-2146.

Sun, V.Z. and Milliken, R.E., 2014. The geology and mineralogy of Ritchey crater, Mars: Evidence for post-Noachian clay formation. Journal of Geophysical Research: Planets, 119(4), pp.810-836.

Sun, V.Z. and Milliken, R.E., 2015. Ancient and recent clay formation on Mars as revealed from a global survey of hydrous minerals in crater central peaks. Journal of Geophysical Research: Planets, 120(12), pp.2293-2332.

Thomas, N., et al. The Colour and Stereo Surface Imaging System (CaSSIS) for the ExoMars Trace Gas Orbiter, submitted to SSR, November 2016

Tornabene, L.L., Moersch, J.E., McSween, H.Y., Hamilton, V.E., Piatek, J.L. and Christensen, P.R., 2008. Surface and crater-exposed lithologic units of the Isidis Basin as mapped by coanalysis of THEMIS and TES derived data products. Journal of Geophysical Research: Planets, 113(E10).

Tornabene, L.L., Osinski, G.R., Barlow, N.G., Bray, V.J., Caudill, C.M., D'Aoust, B., Ding, N., Hopkins, R., Nuhn, A.M., Mayne, A. and McEwen, A.S., 2015. Meter-to Decameter-Scale Characteristics of Central Uplifts Revealed by the Mars Reconnaissance Orbiter. LPI Contributions, 1861, p.1043.

Tulyakov S, Ivanov A, Thomas N, Roloff V, Pommerol A, Cremonese G, Weigel T, Fleuret $\mathrm{F}$ Geometric calibration of Colour and Stereo Surface Imaging System of ESA's Trace Gas Orbiter. ArXiv e-prints, (2017). 1707.00606

Unsalan, Cem., and K. L. Boyer. (2004). A system to detect houses and residential street networks in multispectral satellite images. Proceedings of the 17th International Conference on Pattern Recognition (ICPR '04), Volume 3.

Vago, J., O. Witasse, H. Svedhem, P. Baglioni, A. Haldemann, G. Gianfiglio, T. Blancquaert, D. McCoy, and R. de Groot (2015), ESA ExoMars program: The next step in exploring Mars, Solar System Research, 49, 518-528.

Villanueva, G. L., Mumma, M. J., Novak, R. E., Radeva, Y. L., Käufl, H. U., Smette, A., ... \& Hartogh, P. (2013). A sensitive search for organics $(\mathrm{CH} 4, \mathrm{CH} 3 \mathrm{OH}$, $\mathrm{H} 2 \mathrm{CO}, \mathrm{C} 2 \mathrm{H} 6, \mathrm{C} 2 \mathrm{H} 2, \mathrm{C} 2 \mathrm{H} 4)$, hydroperoxyl ( $\mathrm{HO} 2)$, nitrogen compounds $(\mathrm{N} 2 \mathrm{O}, \mathrm{NH} 3, \mathrm{HCN})$ and chlorine species $(\mathrm{HCl}, \mathrm{CH} 3 \mathrm{Cl})$ on Mars using groundbased high-resolution infrared spectroscopy. Icarus, 223(1), 11-27.

Vincendon, M., 2015. Identification of Mars gully activity types associated with ice composition. J. Geophys. Res. Planets 120, 1859-1879. doi:10.1002/2015JE004909Viviano, C.E., Moersch, J.E. and McSween, H.Y., 2013. Implications for early hydrothermal environments on Mars through the spectral evidence for carbonation and chloritization reactions in the Nili Fossae region. Journal of Geophysical Research: Planets, 118(9), pp.1858-1872.

Viviano-Beck, C.E., Seelos, F.P., Murchie, S.L., Kahn, E.G., Seelos, K.D., Taylor, H.W., Taylor, K., Ehlmann, B.L., Wiseman, S.M., Mustard, J.F. and Morgan, M.F., 2014. Revised CRISM spectral parameters and summary products based on the currently detected mineral diversity on Mars. Journal of Geophysical Research: Planets, 119(6), pp.1403-1431.

Webster, C. R., Mahaffy, P. R., Atreya, S. K., Flesch, G. J., Mischna, M. A., Meslin, P. Y., ... \& Martín-Torres, J. (2015). Mars methane detection and variability at Gale crater. Science, 347(6220), 415-417. 
2407 Williams Jr, R. S., \& Ferrigno, J. G. (1988). Satellite image atlas of glaciers of the world (No. 1386). US Geological Survey.

Wolff, M. J., Smith, M. D., Clancy, R. T., Arvidson, R., Kahre, M., Seelos, F., ... \& Savijärvi, H. (2009). Wavelength dependence of dust aerosol single scattering albedo as observed by the Compact Reconnaissance Imaging Spectrometer. Journal of Geophysical Research: Planets, 114(E2).

Wulf, G., Poelchau, M.H. and Kenkmann, T., 2012. Structural asymmetry in martian impact craters as an indicator for an impact trajectory. Icarus, 220(1), pp.194-204.

Wray, J.J., Ehlmann, B.L., Squyres, S.W., Mustard, J.F. and Kirk, R.L., 2008. Compositional stratigraphy of clay-bearing layered deposits at Mawrth Vallis, Mars. Geophysical Research Letters, 35(12).

Wray, J.J., Murchie, S.L., Bishop, J.L., EhImann, B.L., Milliken, R.E., Wilhelm, M.B., Seelos, K.D. and Chojnacki, M., 2016. Orbital evidence for more widespread carbonate-bearing rocks on Mars. Journal of Geophysical Research: Planets, 121(4), pp.652-677.

Wray, J.J. and EhImann, B.L., 2011. Geology of possible Martian methane source regions. Planetary and Space Science, 59(2), pp.196-202.

Wyatt, M.B. and McSween, H.Y., 2002. Spectral evidence for weathered basalt as an alternative to andesite in the northern lowlands of Mars. Nature, 417(6886), pp.263-266.

Zurek, R.W. and Smrekar, S.E., 2007. An overview of the Mars Reconnaissance Orbiter (MRO) science mission. Journal of Geophysical Research: Planets, 112(E5).

Zurek, R. W., Chicarro, A., Allen, M. A., Bertaux, J. L., Clancy, R. T., Daerden, F., ... \& Smith, M. D. (2011). Assessment of a 2016 mission concept: The search for trace gases in the atmosphere of Mars. Planetary and Space Science, 59(2), 284-291. 


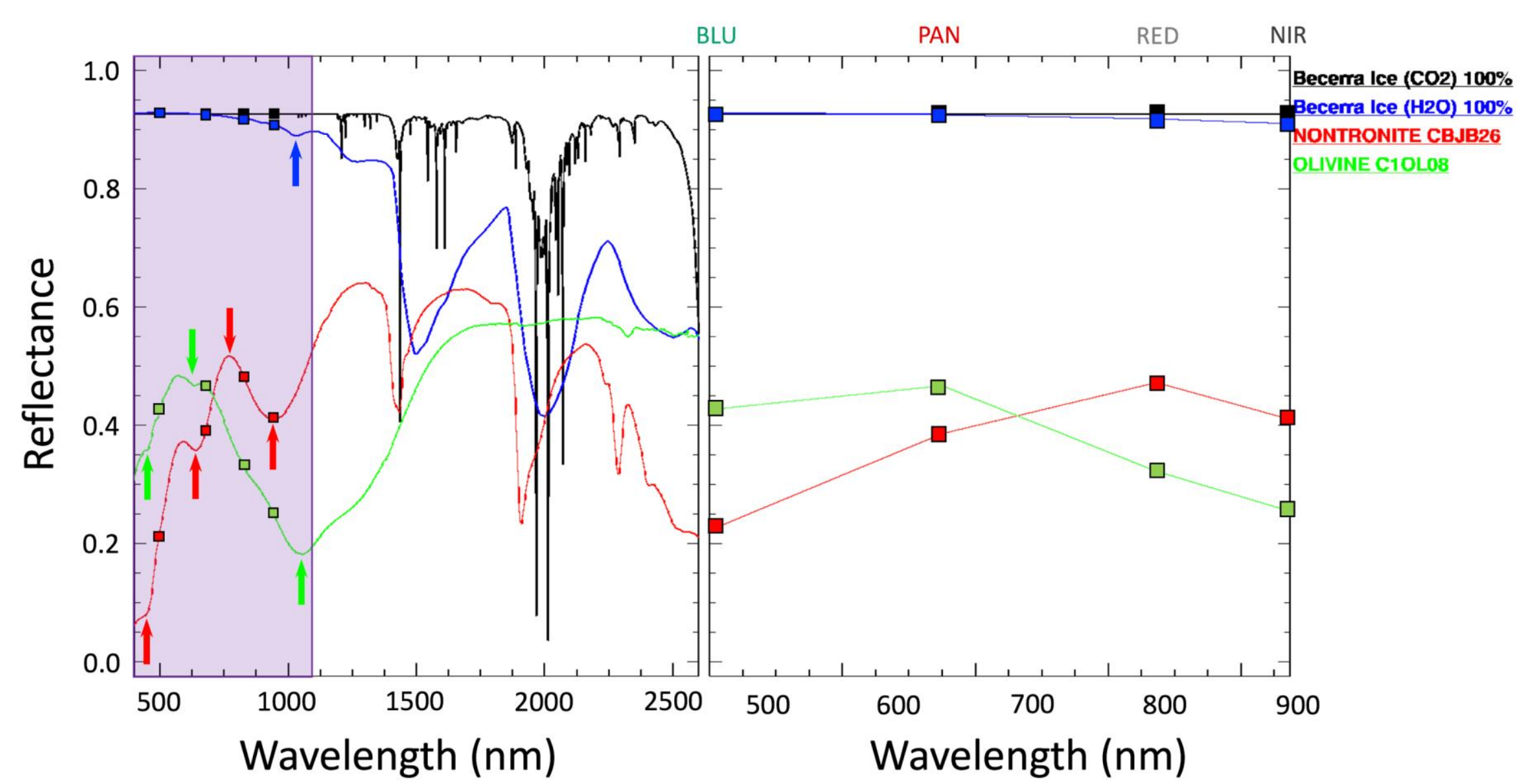

Figure 1 Selected ferric $\left(\mathrm{Fe}^{3+}\right.$ ) iron-bearing (nontronite - ID\# CBJB26) and ferrous ( $\mathrm{Fe}^{2+}$ ) iron-rich (olivine - ID\# C10L08) mineral spectra (300 bands) from the CRISM spectral library (left) and Hapke-modeled cos (i)-corrected I/F ice spectra (750 bands) for pure $\mathrm{CO}_{2}$ and $\mathrm{H}_{2} \mathrm{O}$ at an incidence of $68^{\circ}$ (after Beccera et al 2015) compared to their 4-point CaSSIS convolved equivalents (right). Arrows show the general location of key diagnostic spectral features, which the CaSSIS bands (squares) would be sensitive to. The shaded region on the left denotes the instrument filter response range of CaSSIS ( $\sim 00-1100 \mathrm{~nm} ;$ c.f., Figure 2 and see Thomas et al this issue). 


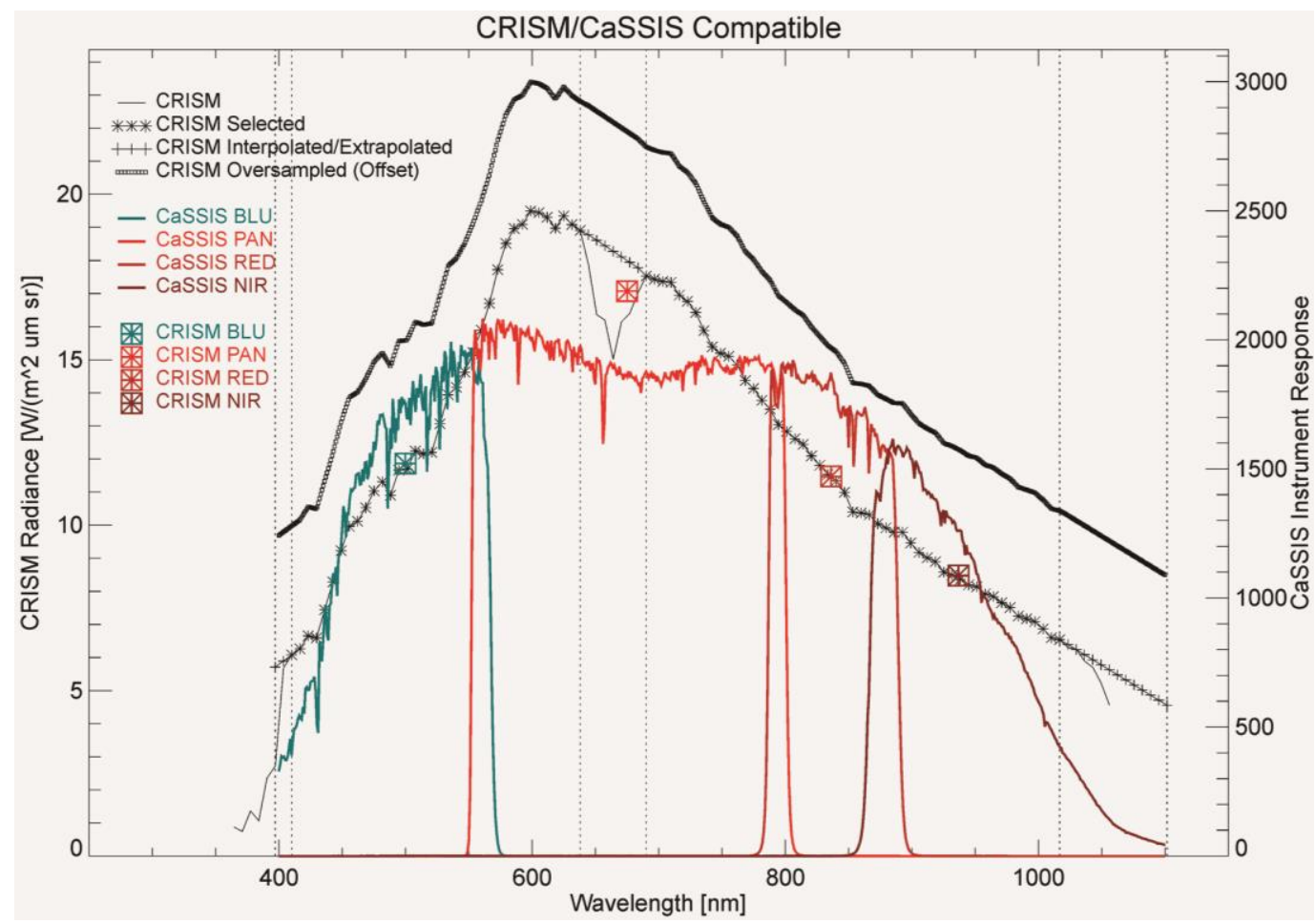

Figure 2 CRISM-CaSSIS compatible spectral processing. CRISM FRT00003E12 median radiance spectrum at native spectral sampling (unadorned black line), selected channels (black asterisks) with interpolated and extrapolated points (black crosses), and oversampled (black squares offset), CaSSIS instrument response functions (coloured lines - refer to alternate axis on right), and CRISM/CaSSIS compatible radiance values (large coloured symbols). Vertical dotted lines indicate interpolation and extrapolation wavelength boundaries. Each CRISM VNIR I/F spectrum in the input image cube is transformed to spectral radiance, interpolated/extrapolated to cover the full spectral range of the CaSSIS response, oversampled to the CaSSIS response spectral sampling, integrated by the response functions, and the result transformed back to I/F. 

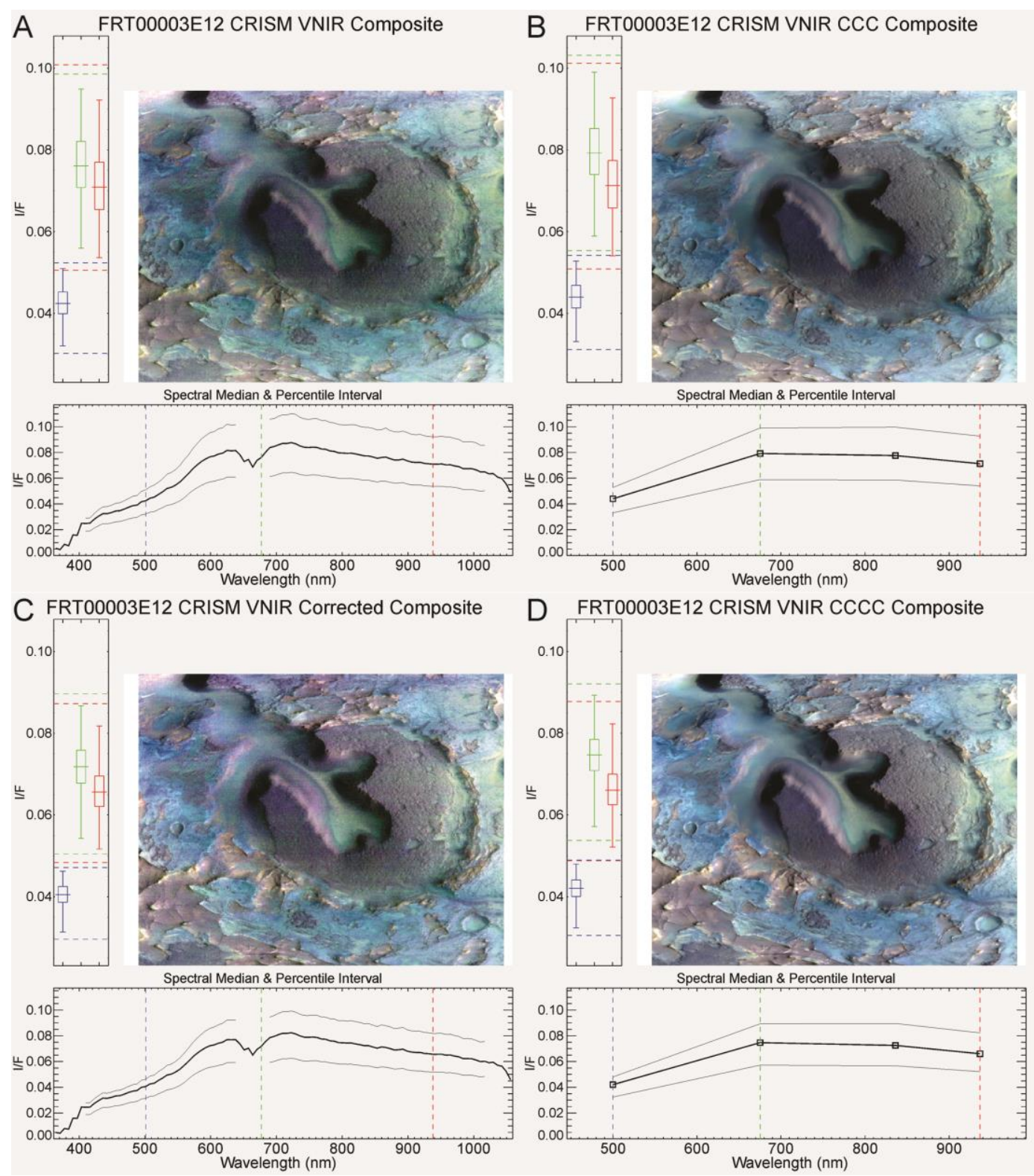

Figure 3 CRISM-CaSSIS Compatible $\left(C^{3}\right)$ and Corrected CRISM-CaSSIS Compatible $\left(C^{4}\right)$ processing progression presented as a series of 3-panel plots for CRISM observation FRT00003E12. Each 3panel plot is composed of a sensor space (non-map projected) RGB composite ( $10-12 \mathrm{~km}$ in width) with a $0.25 \%$ linear stretch on each band, a spectral plot with the median spectrum for the scene and a 1-99\% spectral envelope, and box-plots illustrating the data distribution of the RGB composite bands. The vertical dashed lines on the spectral plot indicate the spectral bands that are mapped into the RGB composite channels, and the horizontal dashed lines on the box-plots indicate the stretch limits applied to each RGB composite band. (A) The RGB composite bands are individual CRISM channels that closely match the effective wavelengths of the CaSSIS bandpass (see Table 1). Note that effective wavelength of the CaSSIS PAN band ( $675 \mathrm{~nm}$ ) is in the spectral region affected by the CRISM VNIR filter boundary. (B) Visualization of the corresponding CRISMCaSSIS Compatible $\left(\mathrm{C}^{3}\right)$ data product. The spectrum for each spatial pixel in $(\mathbf{A})$ has been processed to CaSSIS 4-band colour as described in the text. (C) CRISM observation FRT00003E12 after application of the Empirical Geometric Normalization. (D) Corrected CRISM-CaSSIS Compatible ( ${ }^{4}$ ) data product (simulated CaSSIS colours at $18-20 \mathrm{~m} /$ pixel). The spectrum for each spatial pixel in (C) has been processed to CaSSIS 4-band colour as described in the text. 


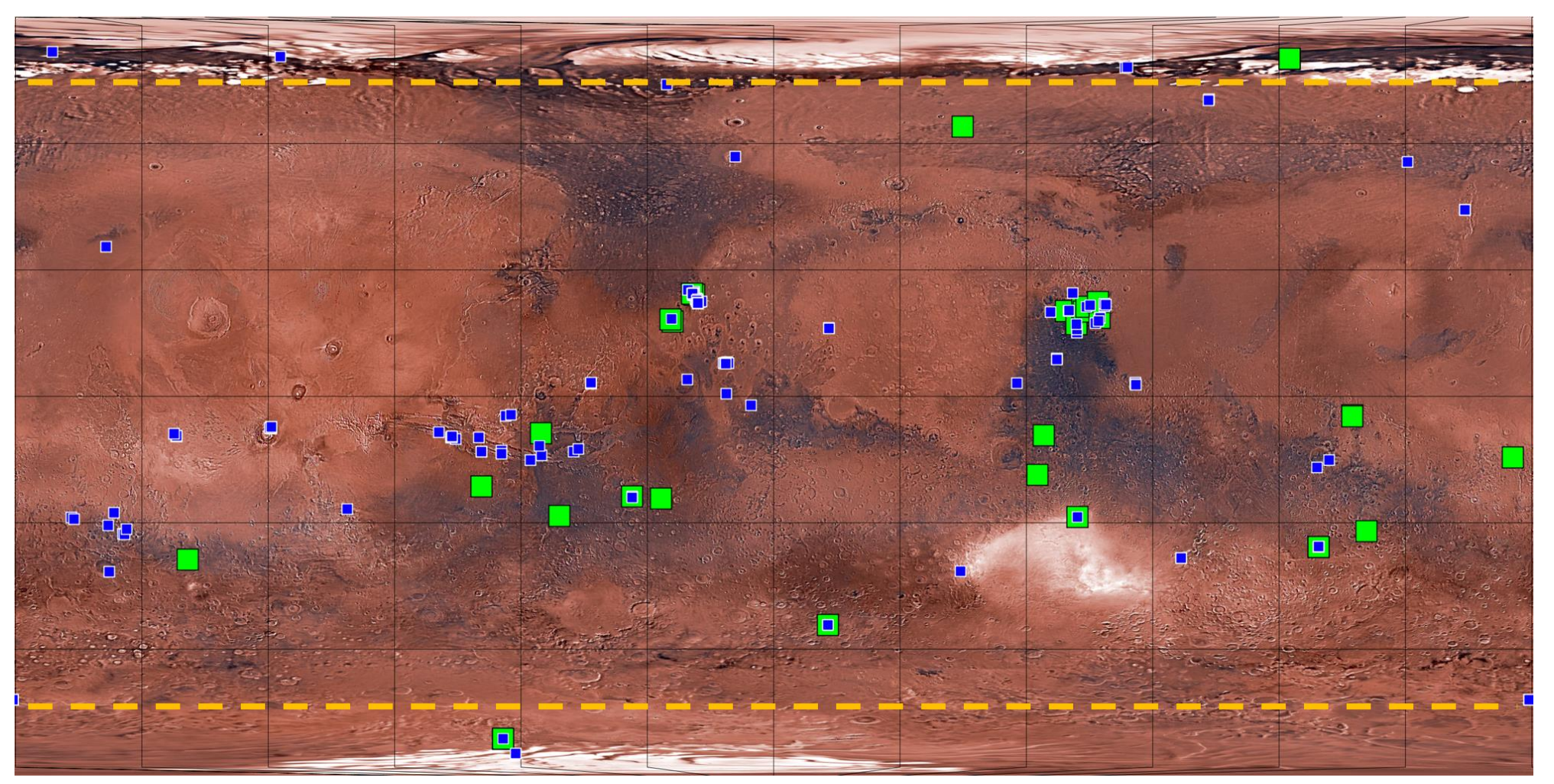

Figure 4 Map of target locations of 180 simulated CaSSIS images on Mars. Green squares denote the locations of the 33 "fully"-simulated CaSSIS image cubes, which includes 30 unique locations; while the smaller blue squares indicate the locations of the CRISM-CaSSIS compatible colour only products (i.e., the $\mathrm{C}^{3}$ and $\mathrm{C}^{4} \mathrm{CRISM}$-derived cubes), which retain the spatial resolution of the CRISM cube from which they were derived ( $18-36 \mathrm{~m} / \mathrm{pixel})$. This includes several of the CRISM mineral/phase localities reported in VivianoBeck et al (2014). The location map uses the Viking colour MDIM 2.1. mosaic to show that the simulations are selected from diverse surfaces ranging in brightness and ferric/ferrous content. The map also shows that our simulated CaSSIS images cover diverse surfaces across a wide latitudinal and longitudinal range. The dashed-line denotes the $74^{\text {th }}$ parallel in both hemispheres marking the approximate boundary of CaSSIS observations based on its orbital inclination. See Table S1 and the associated help file included with our supporting online supplementary materials for more information on the 181 simulated locations (180 surface locations plus one of Phobos). Background image credits: JPL/NASA and USGS/NASA-AMES. 


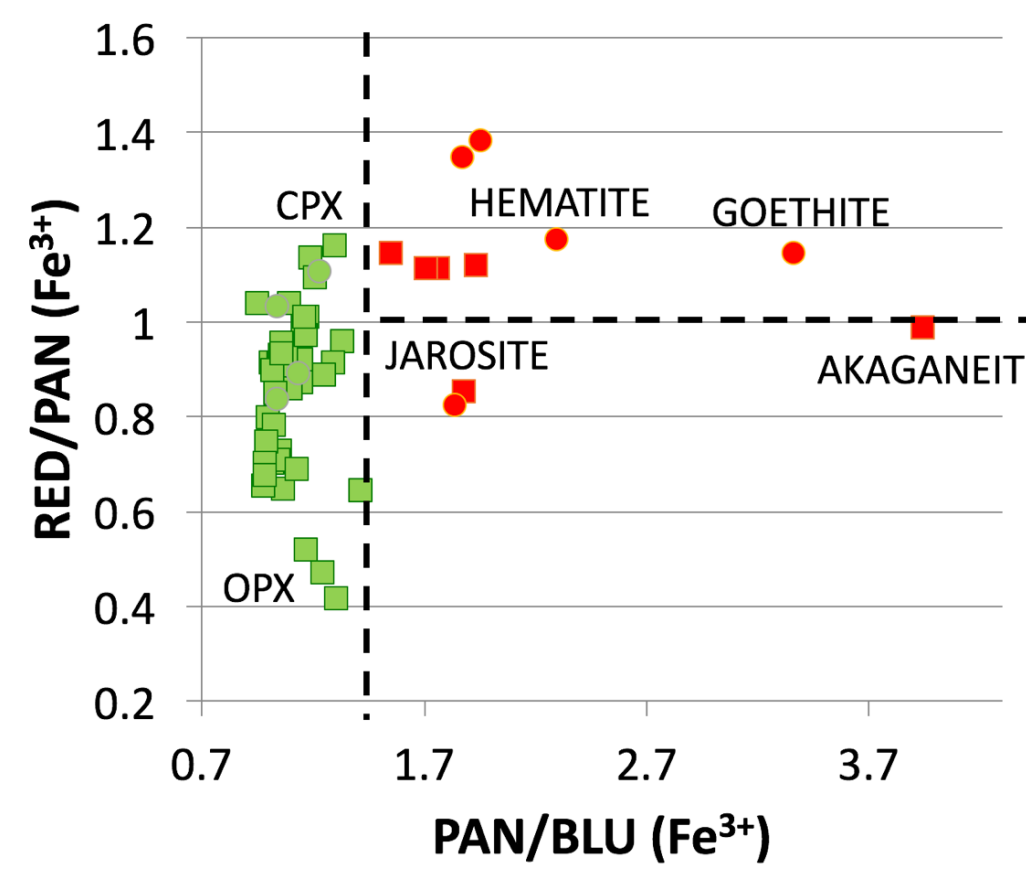

$\square$ CRISM Lib: Ferrous

- CRISM Lib: Ferric

USGS Lib: Ferrous

- USGS Lib: Ferric

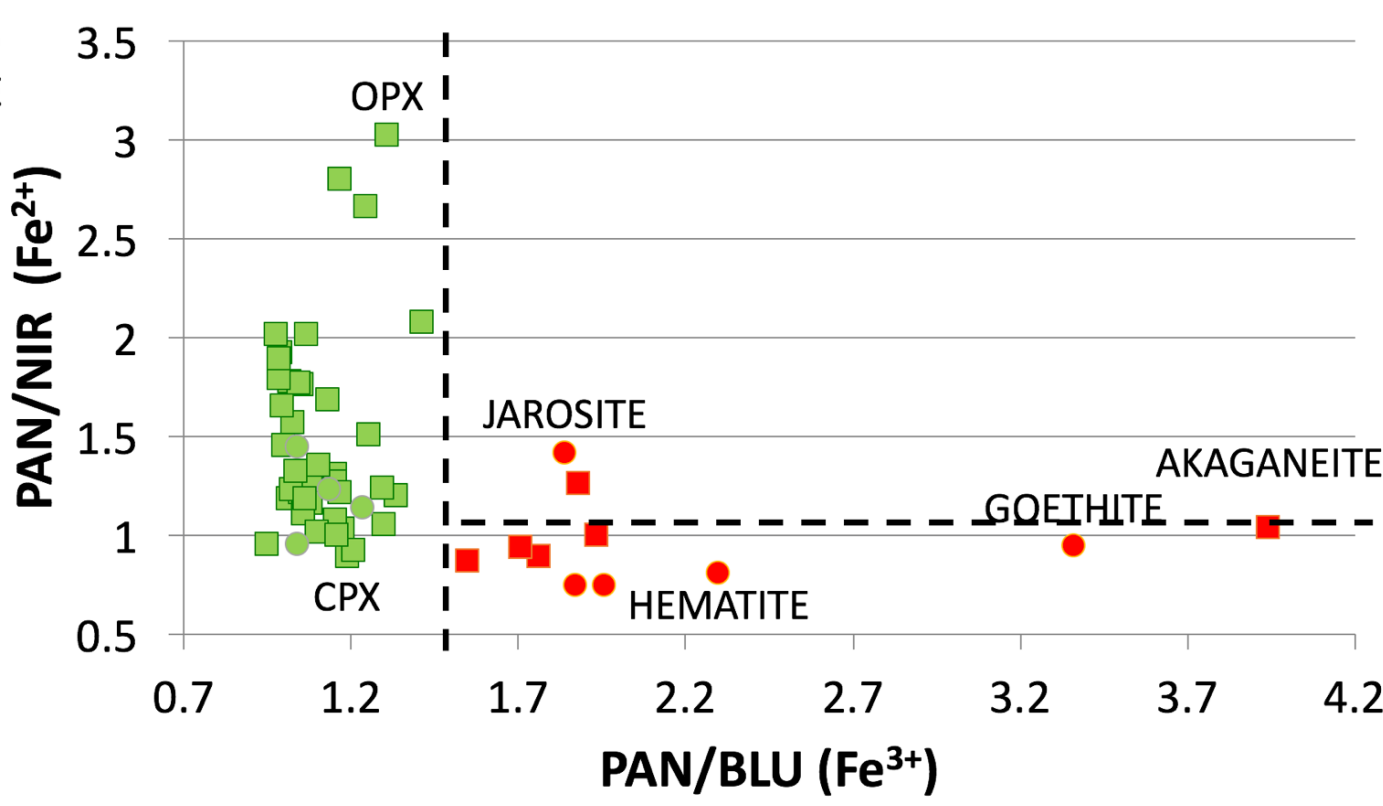

Figure 5 Band ratio bivariate plots of CaSSIS instrument response-convolved laboratory spectra of selected ferrous ( $\left(\mathrm{Fe}^{2+}\right)$ iron (green) and ferric $\left(\mathrm{Fe}^{3+}\right)$ iron $(\mathrm{red})$ bearing minerals from the USGS and CRISM team spectral mineral libraries (Clark et al 1993; http://speclib.rsl.wustl.edu/). The plot indicates that 2PAN/1BLU ratios fully discriminate ferrous from ferric minerals, with all ferric minerals generally having 2PAN/1BLU values of $<1.41$ (most $<1.3$ ); and all ferric with 2PAN/1BLU values $>1.55$ (most $>1.7$ ). The 2PAN/4NIR does a reasonable job of discriminating ferrous from ferric minerals (right), with most ferrous materials having values generally $>1$ and most ferric materials with values $<1$. The 3RED/2PAN provides additional discrimination of ferric minerals (left). Dashed lines demark the general ferrous vs. ferric domains (See text and Table 3 for additional information on these ratios). 


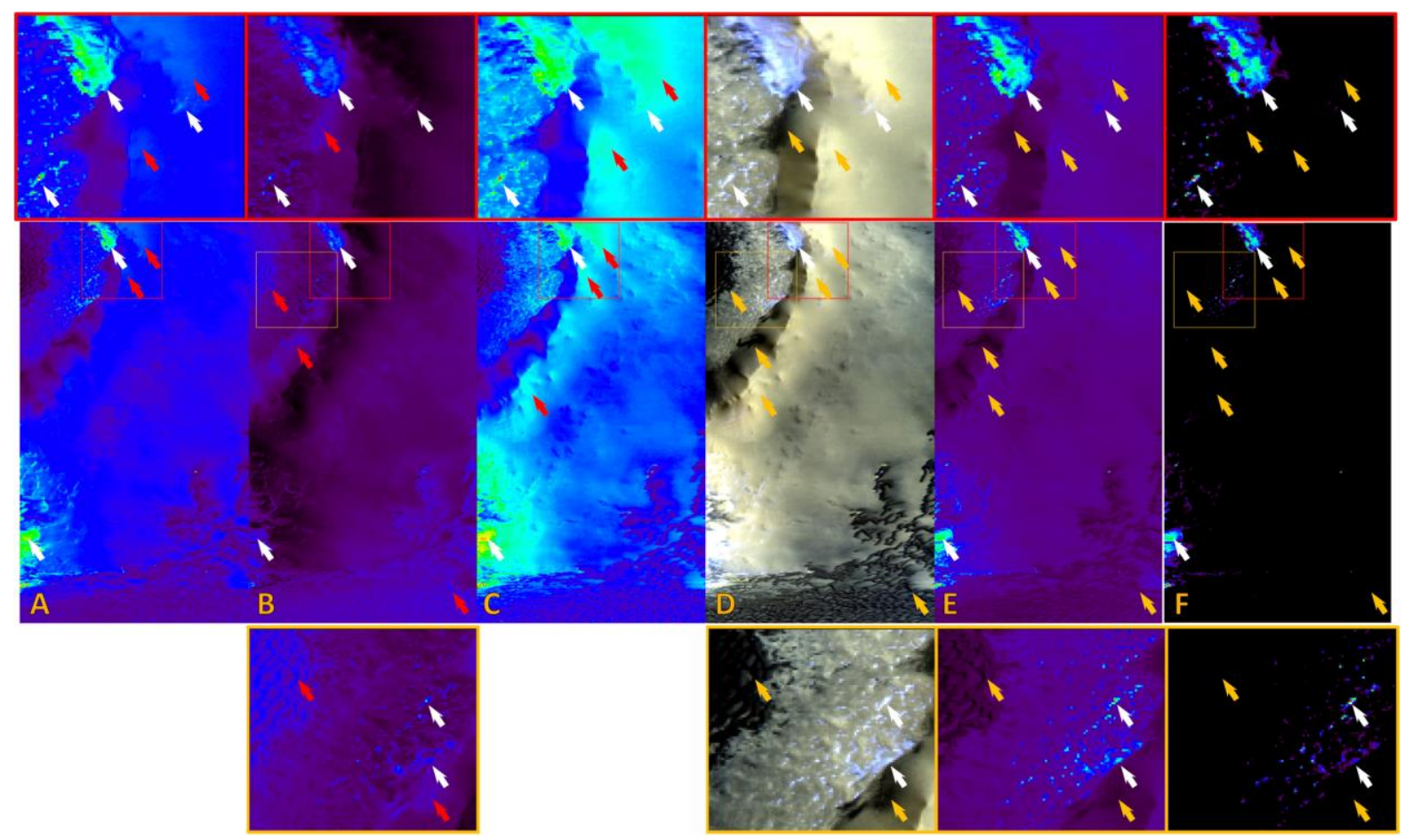

Figure 6 A comparison of a set of simulated CaSSIS products (A-E) to a CRISM IR 1500nm banddepth spectral parameter image (i.e., BD1500) for $\mathrm{H}_{2} \mathrm{O}$ ice (F). The CRISM cube used here is derived from S-detector data and the targeted observation HRL00002885, which covers the Viviano-Beck et al (2014) CRISM mineral/phase locality for $\mathrm{H}_{2} \mathrm{O}$ ice. White arrows are locations of positive $\mathrm{H}_{2} \mathrm{O}$ ice detections, while red arrows are negative detections (based on [F]). Comparing these two sets of arrows in (A-C) highlights the ambiguities between ice and non-ice-bearing surfaces in the first 3 panels. Orange arrows (in D-F) are used to indicate where false-detections occurred in (A-C), but are not ambiguous in images (D-F). Red and orange boxes in panels (A-F) correspond to the colour-coded rows of $3 x$ zoomed subimages at the top and bottom. A) This colourized simulated CaSSIS BLU band image shows strongly illuminated (red arrows) areas that are ambiguous with ices. B) This colourized simulated CaSSIS NIR/BLU ratio image shows basaltic dunes (red arrows) that are ambiguous with ices. C) This colourized simulated CaSSIS mean multiband reflectance or I/F intensity image also shows illuminated areas (red arrows) that are ambiguous with ices. D) Shows a simulated CaSSIS infrared colour image (IRB1) for context. E) This colourized simulated CaSSIS image produced by dividing panels (C) by (B) and most effectively highlights surface ice and frost (c.f., with (F)). (A-C, E) all have linear min-max stretches and an ENVI pre-defined BLUE-RED colour table applied, while (D) has a linear $0.5 \%$ stretch applied to each band, and (F) has a linear stretch from a value of 0 to its maximum value of 0.25. Image credits: WesternU-CPSX/ASC-CSA/NASA, NASA/JPL/JHU-APL and ESA/Roscosmos/ExoMars/CaSSIS. 


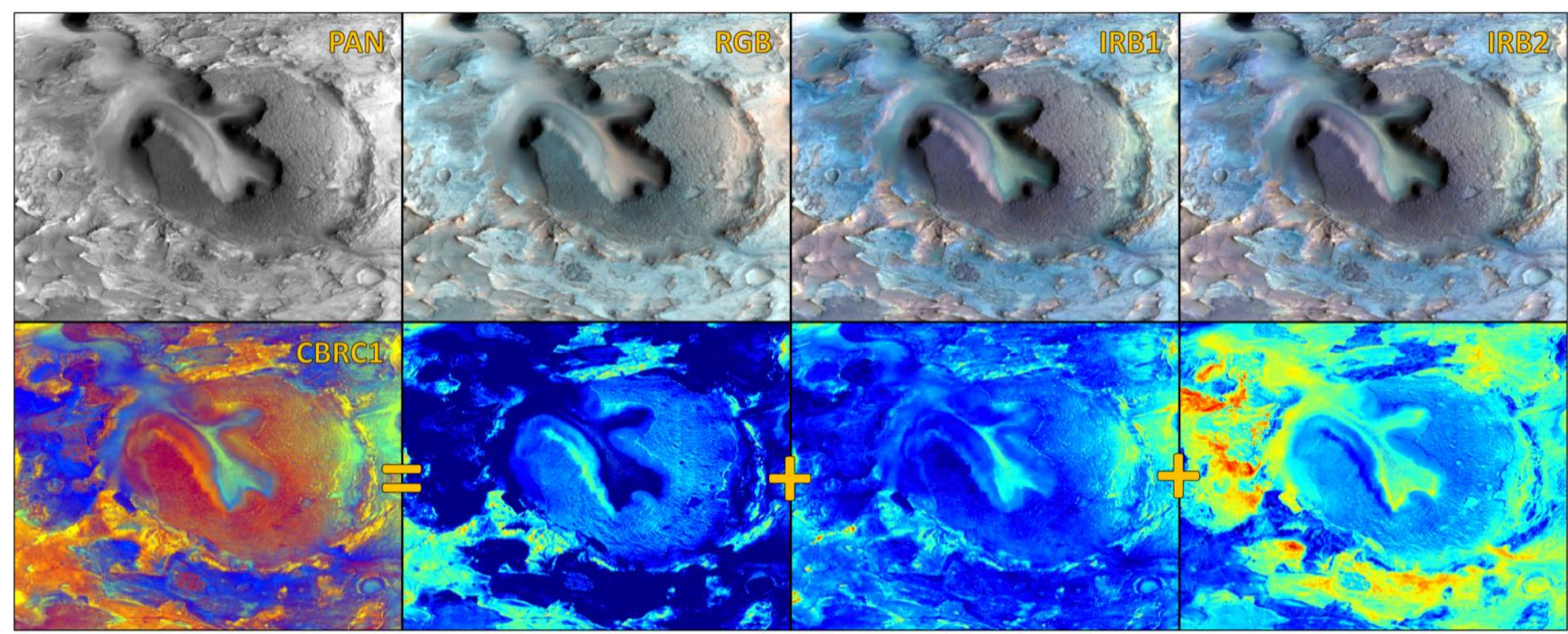

Ferric - $\mathrm{Fe}^{3+} 836 / 675 \mathrm{~nm}$

Ferric $-\mathrm{Fe}^{3+} 675 / 500 \mathrm{~nm}$

Ferrous - $\mathrm{Fe}^{2+}$ 675/937 $\mathrm{nm}$

Figure $7 \mathrm{~A}$ set of suggested CaSSIS image modes/colour-products based on a non-map-projected ( $\sim 10-12 \mathrm{~km}$ in width) $\mathrm{C}^{4}$ cube produced from S-detector data from the targeted observation FRT00003E12, centred at $77.1^{\circ} \mathrm{E}, \mathrm{E}, 22.24^{\circ} \mathrm{N}$, in the Nili Fossae region. PAN = Greyscale red image using the 2PAN Band; RGB = Synthetic "true" colour (bands 2-1-0); IRB1 = Infrared colour image using the last NIR band - 4NIR (Bands 4-2-1); IRB2 = Infrared colour image using the first NIR band - 3RED (Bands 3-2-1); (bottom) CBRC1 = band ratios using 3RED/2PAN, 2PAN/1BLU, 2PAN/4NIR in R-G-B. The last 3 images in the bottom row are the individual band ratios of which CBRC1 at the beginning of the row is comprised (in R-G-B order). Each of these greyscale images have a colour table applied to them to show areas with low and high values (i.e., variations of relative band-strength as a proxy for exposure or concentration of Fe-bearing species). The top row, and the CBRC1, images all have a linear $0.5 \%$ stretch on each band, while the last three images in the bottom row all have linear min-max stretches to the upper part of their histograms and an ENVI pre-defined BLUE-RED colour table applied. Image credits: WesternU-CPSX/ASC-CSA/NASA, NASA/JPL/JHU-APL and ESA/Roscosmos/ExoMars/CaSSIS. 

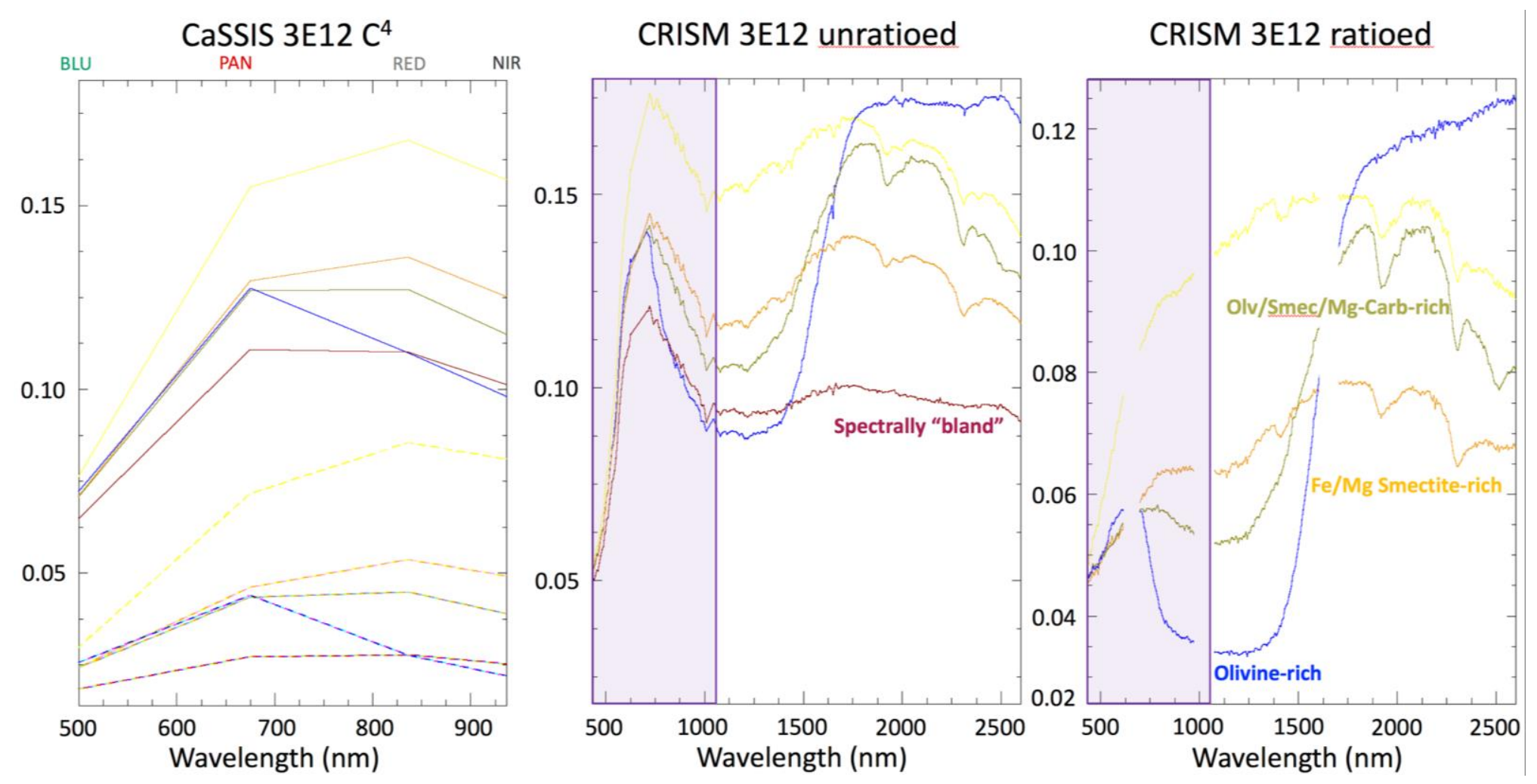

Figure 8 Simulated CaSSIS C 4 image-derived averaged spectra ( $3 \times 3$ pixel) compared to CRISM spectra derived from a "joined" CRISM MTRDR cube. Locations of the averaged spectra were based on spectral/colour units defined off our CBRC1 composite presented in Figure 7, and the known location for Mg-carbonate bearing deposits, reported by EhImann et al (2008) and Viviano-Beck et al (2014), in the scene. The sensitivity range of the 4 colour bands of CaSSIS is represented by the shaded regions shown in the two plots on the right. The set of coloured 4-point simulated CaSSIS spectra (left) are extracted from a C cube created from CRISM targeted observation FRT00003E12, with the lower I/F coloured set of spectra (dashed lines) originating from an ad hoc atmospheric/haze-corrected cube using a dark subtraction method. These simulated CaSSIS spectra are compared to unratioed (centred) and ratioed (right) spectra extracted from the joined CRISM MTRDR cube. The olivine- and smectite-rich spectra (right) are consistent with the results of previous studies (e.g., Mustard et al 2009), but can also be compared to lab spectra presented in Figure 1. 


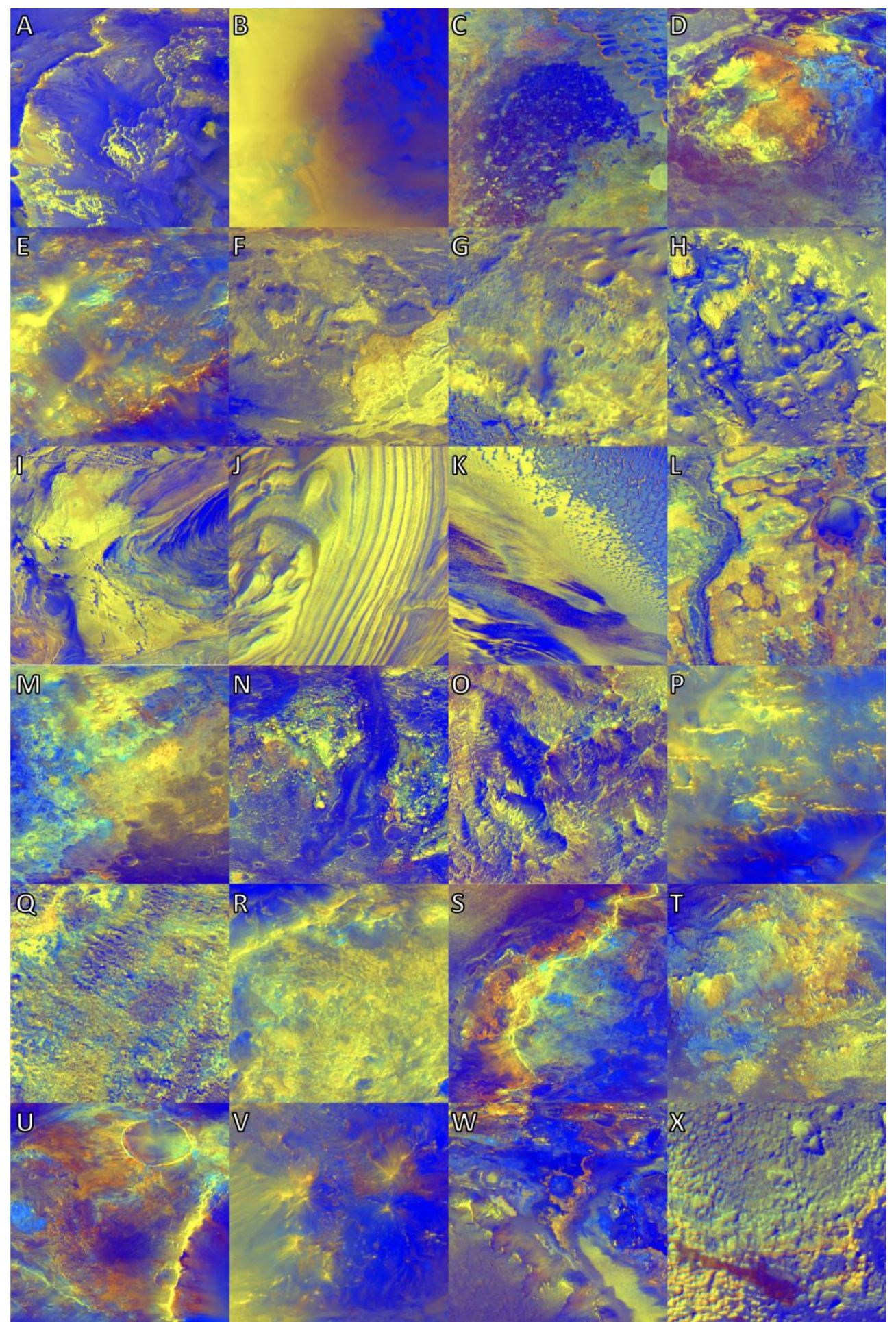

Figure 9 Cropped examples of simulated CaSSIS CBRC1s produced from non-map projected $\mathrm{C}^{4}$ cubes based off the CRISM targeted observations covering 24 of the Viviano-Beck et al (2014) CRISM mineral/spectral-type localities on Mars. Starting from the top and moving to the right, these include the type localties for: (A) Hematite (B385), (B) Mg-Olivine (C26C), (C) Plagioclase (82EE), (D) Low-Ca Pyroxene (64D9), (E) High-Ca Pyroxene (8F68), (F) Monohydrated sulfate (A91C), (G) Alunite (B252), (H) Hydroxlated Fe-sulfate (98B2), (I) Jarosite (13F5B), (J) Polyhydrated sulfate (9COA), (K) Gypsum (CA5C), (L) Bassanite (9326), (M) Kaolinite (ABCB), (N) Al-smectite (3BFB), (O) Margarite \& Serpentine (634B), (P) Illite/Muscovite(454E), (Q) Fe-smectite (A425), (R) Mg-smectite (9365), (S) Talc (9D44) (T) Chlorite \& Ca/Fe-Carbonate (A546), (U) Prehnite (50F2), (V) Hydrated silica \& Analcime (9312), (W) Epidote (CBE5), and (X) Chloride (AB81). Fe-Olivine \& Mg-Carbonate (3E12) CBRC1 is shown in Figure 7. Image credits: WesternU-CPSX/ASCCSA/NASA, NASA/JPL/JHU-APL and ESA/Roscosmos/ExoMars/CaSSIS. 


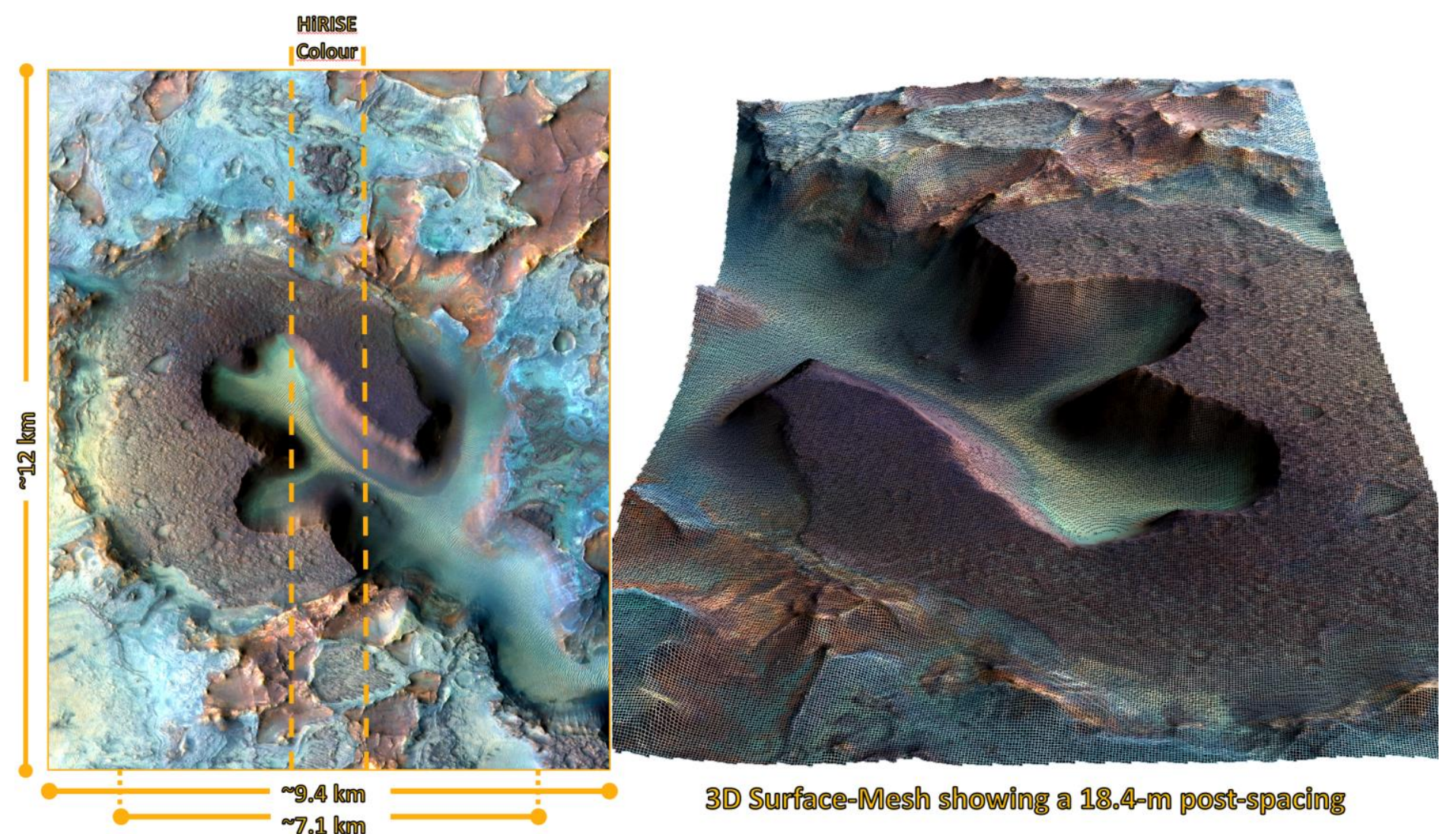

Figure 10 A fully-simulated CaSSIS infrared colour image (IRB1) at $4.6 \mathrm{~m} /$ pixel covering olivine-, serpentine-, smectite- and carbonate-bearing terrain in the Nili Fossae region $\left(77.1^{\circ} \mathrm{E}, \mathrm{E}, 22.24^{\circ} \mathrm{N}\right)$. (Left) The fully-simulated CaSSIS IRB1 image was cropped and annotated to show the two swath width modes currently being planned for CaSSIS, and compared with the maximum colour coverage provided by HiRISE (dashed-lines). The length of cropped image is $\sim 12 \mathrm{~km}$; however, CaSSIS will be able to achieve $\sim 4 x$ this length. (Right) A 3D perspective view (looking southward) made from overlaying the fully-simulated CaSSIS IRB1 image on a HiRISE DTM resampled to the anticipated CaSSIS DTM resolution (18.4 m/post) and set to a vertical exaggeration of 2x. Image credits: WesternU-CPSX/ASC-CSA/NASA, NASA/JPL/JHU-APL/MSSS/UofA and ESA/Roscosmos/ExoMars/CaSSIS. 


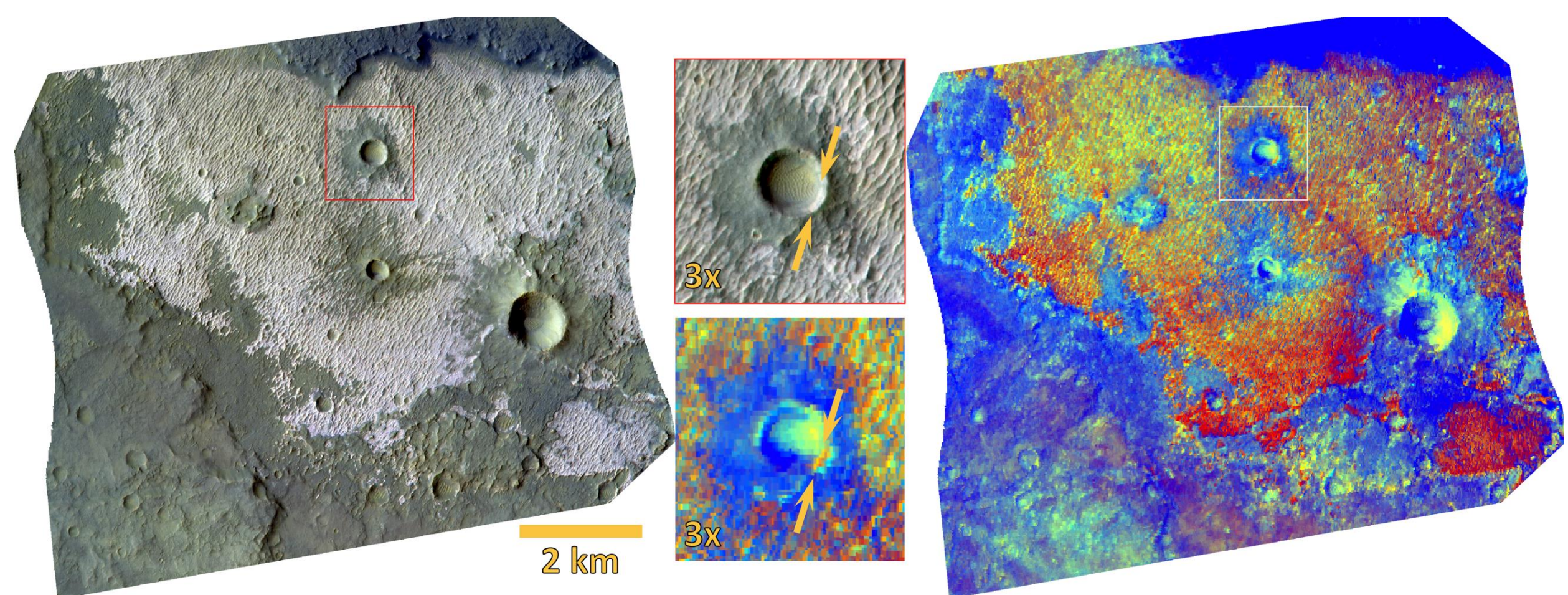

Figure 11 Fully-simulated CaSSIS colour images of a chloride-bearing site $\left(221.04^{\circ} \mathrm{E}, 39.05^{\circ} \mathrm{S}\right)$ documented in the Martian chloride discovery paper (see Osterloo et al 2008$)$. Colour infrared - IRB1 (left), and CBRC1 (right). The zoomed images (centre) highlight a 500-m crater. The colour characteristics of its ejecta, and an exposure in the crater wall, (arrows) suggest that the chloride-bearing deposits are consistent with representing a salt pan or flat (i.e., a superficial surface layer/crust of salts) underlain by ancient lava flows. In the upper right of the image is a more recent lava flow emanating from Daedalia Planum, which possesses a strong Ferrous iron-bearing signature consistent with relatively unaltered mafic-bearing materials. The image is $\sim 9.3 \mathrm{~km}$ across at its narrowest. Image credits: WesternU-CPSX/ASC-CSA/NASA, NASA/JPL/JHU-APL/MSSS/UofA and ESA/Roscosmos/ExoMars/CaSSIS. 


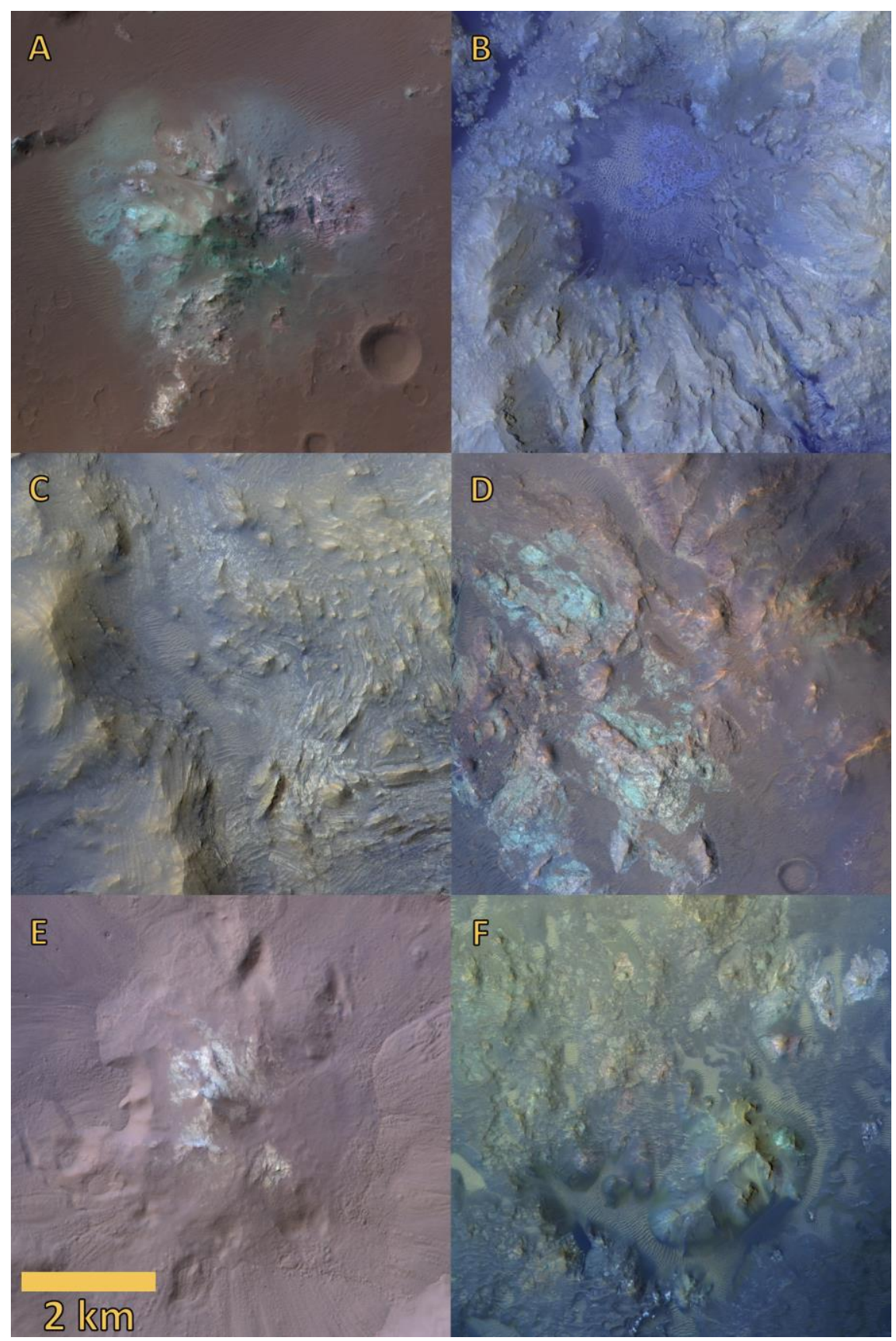

Figure 12 Fully-simulated CaSSIS infrared colour images (IRB1) of various crater central uplifts exposing the bedrock textural types defined and described by Tornabene et al (2015 and references therein): Layered (LB), Fractured and Massive (FMB) and "Mega"-brecciated (MBB). (A) Central peak of Alga crater $\left(333.35^{\circ} \mathrm{E}, 24.34^{\circ} \mathrm{S}\right)$ exposing $\mathrm{FMB}$ with various coatings of impact melt-rich breccia deposits with diverse clast compositions (see Skok et al 2012 and D'Aoust 2015), (B) Peak pit of Elorza crater $\left(304.79^{\circ} \mathrm{E}, 8.75^{\circ} \mathrm{S}\right)$ exposing hydrated silica, LCP, and smectitebearing FMB coated by clast-poor impact melt-bearing deposits (see Hopkins et al 2016), (C) Eastern portion of the of the Martin crater central uplift $\left(290.65^{\circ} \mathrm{E}, 21.42^{\circ} \mathrm{S}\right.$ ) exposing $\mathrm{LB}$ (see Caudill et al 2012 and Wulf et al 2012), (D) and (E) Central peaks of unnamed craters $\left(72.14^{\circ} \mathrm{E}\right.$, $28.64^{\circ} \mathrm{S}$ and $72.14^{\circ} \mathrm{E}, 28.64^{\circ} \mathrm{S}$ ) with well-exposed FMB. (F) Central peak complex of Verlaine crater $\left(64.14^{\circ} \mathrm{E}, 9.25^{\circ} \mathrm{S}\right)$ exhibiting partial exposures of both $\mathrm{FMB}$ and $\mathrm{MMB}$ including coatings and flows of diverse impact melt-bearing deposits. (see D'Aoust 2015). Note: the scale bar is on the order of 2x the width of the HiRISE colour swath. Image credits: WesternU-CPSX/ASC-CSA/NASA, NASA/JPL/JHU-APL/MSSS/UofA and ESA/Roscosmos/ExoMars/CaSSIS 


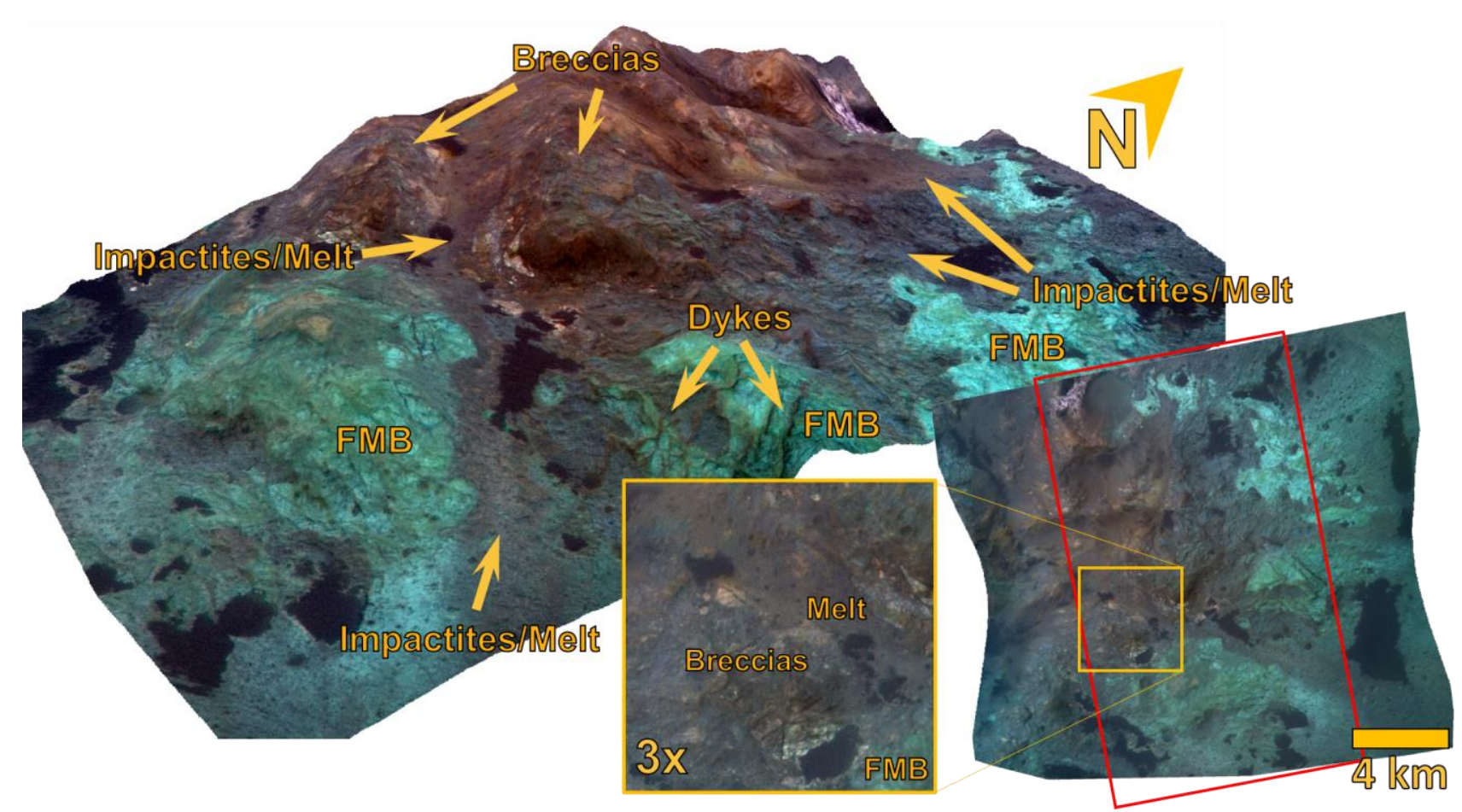

Figure 13 A 3D perspective of fully-simulated CaSSIS infrared colour image (IRB1) covering the eastern portion of the central peak of Ritchey crater $\left(309.15^{\circ} \mathrm{E},-28.45^{\circ} \mathrm{N}\right)$. The red box covers an area in 2D that corresponds to the approximate area covered by the 3D perspective image. Fractured-Massive Bedrock (FMB) is particularly well-exposed in the lower slopes of the uplift (cyan), while the upper slopes appear to be comprised of a "mega" brecciated core exhibiting mostly white or light yellow clasts that are predominately draped by relatively thin dark-toned deposits of melts and breccias. A spectacular continuous flow deposit of impact melt-bearing materials is observed starting at the summit near the annotation "Breccias" and continues down slope beyond the annotation "Impactites/Melt" and conforms with the local topography over its entire extent. Dark-toned lineaments are interpreted to be dykes, which can be seen cross-cutting a well-exposed block of FMB in the eastern flank of the uplift. Based on full-CRISM spectral characteristics, the cyan-coloured bedrock is consistent with LCP-bearing bedrock, limited exposures or clasts of olivine-bearing rocks are white or light yellow, and altered bedrock is magenta in colour. For more details on the Ritchey crater uplift, see Sun and Miliken (2014) and Ding et al (2015). Image credits: WesternU-CPSX/ASCCSA/NASA, NASA/JPL/JHU-APL/MSSS/UofA and ESA/Roscosmos/ExoMars/CaSSIS 


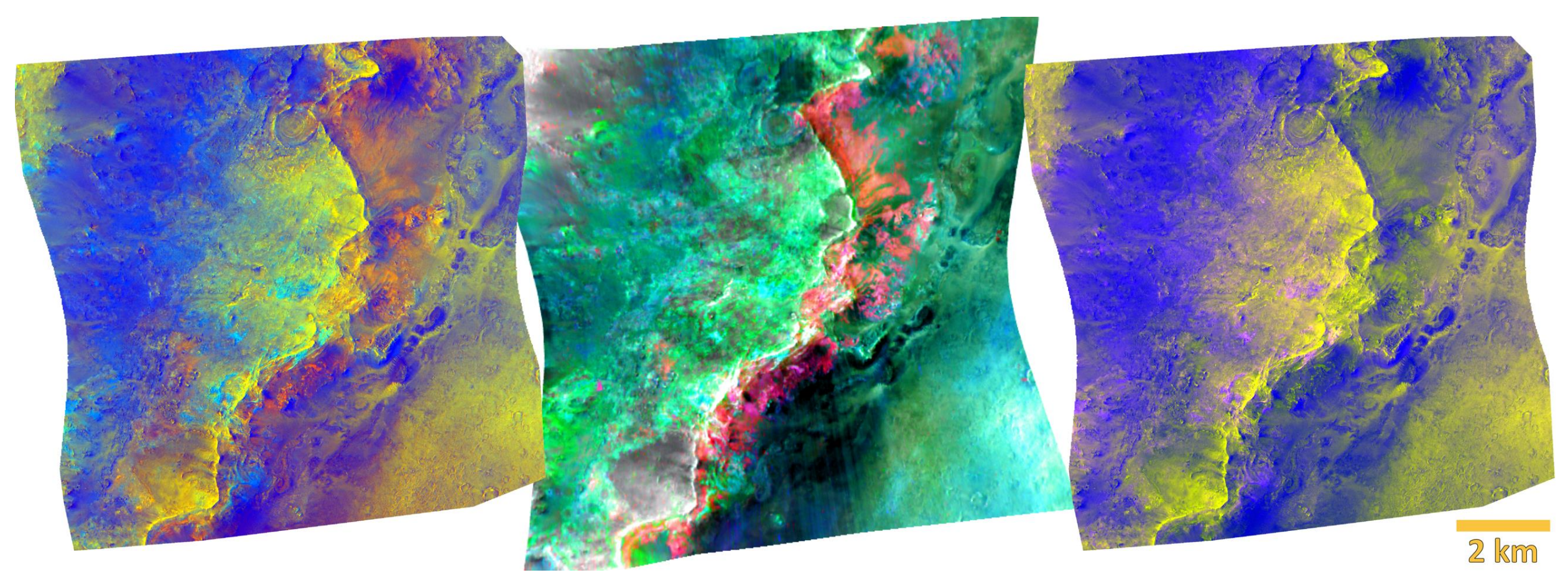

Figure 14 Fully-simulated CaSSIS colour images of the talc CRISM spectral mineral/phase type-locality (Viviano-Beck et al 2013, 2014) centred at $73.68^{\circ} \mathrm{E}, 20.06^{\circ} \mathrm{N}$ in the Nili Fossae region. Here we compare a simulated CaSSIS CBRC1 image that utilizes all 4 bands (left) with a HiRISE-equivalent CBRC image (right) that uses only three bands (see Delamere et al 2010). A simulated CaSSIS 4 band-derived CBRC1, which shows a greater variety of colours, to a 3 band-derived HiRISE CBRC. The colours of the simulated CaSSIS CBRC1 correlate better with the CRISM spectral parameter composite image (centre), which was constructed by combining the standard CRISM-derived "phyllosilicate" (PHY1) and "mafic" (MAF1) spectral parameter images, and then overlain on a CRISM IR brightness image (IRA; 1330 nm). Viviano-Beck et al (2013) have shown CRISM spectral characteristics consistent with a talc-bearing unit that overlies a chlorite-bearing unit within the scarp-face exposed in this scene. These two notable mineral-bearing units are reddish-orange and yellow, respectively, in the simulated CaSSIS CBRC1 (left); these two units only show up as a single coloured unit (yellowish) in the HiRISE CBRC (right). Image credits: WesternU-CPSX/ASC-CSA/NASA, NASA/JPL/JHU-APL/MSSS/UofA and ESA/Roscosmos/ExoMars/CaSSIS. 


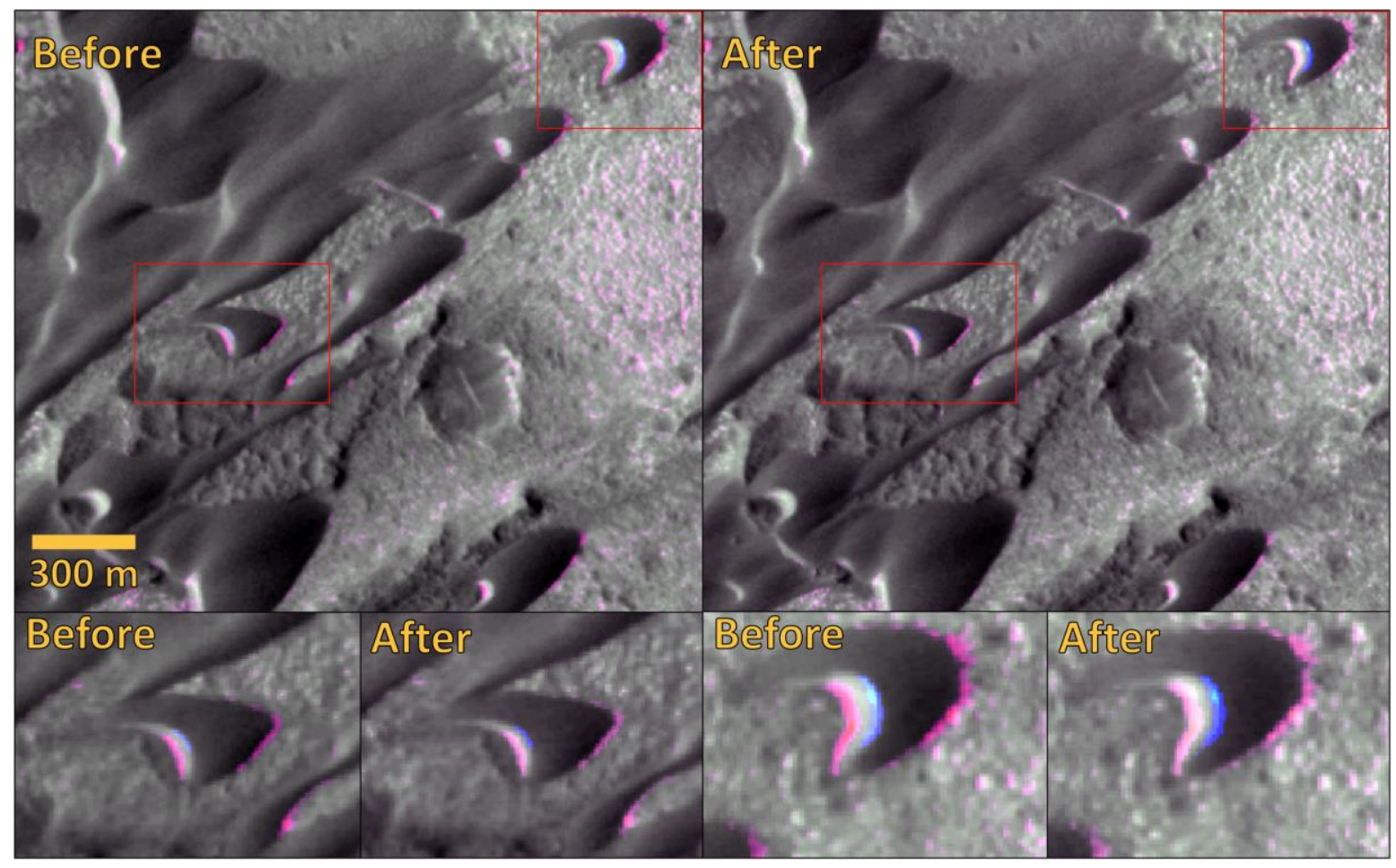

Figure 15 Nili Patera dune field (centered at $67.3^{\circ} \mathrm{E}, 8.8^{\circ} \mathrm{N}$ ) movement recorded by two orthorectified CTX images taken 7.5 years apart and oversampled to the CaSSIS pixel-scale $(4.6 \mathrm{~m} / \mathrm{pix})$. The colour overlay here was created by taking the normalized difference between the two images P04_002427_1888_XI_08N292W (2/1/2007) and F05_037792_1891_XN_09N292W (8/19/2014), and highlights pixels with drastic changes in their intensity values (i.e., at the lower and upper areas of the image histogram). Magenta highlights pixels that move from the lowest parts of the histogram in the "before" image to the highest parts (darker to lighter) in the "after" image, and blue highlights pixels that move from the highest parts of the histogram to the lowest parts (lighter to darker). Highlightedchanges are a consequence of dune migration, which is readily observed in animated GIFs provided as online supplemental materials. This new technique for highlighting changes between images in this fashion is described in more detail in the supporting online supplementary materials included with this manuscript. Image credits: WesternU-CPSX/ASC-CSA/NASA, NASA/JPL/MSSS/UofA and ESA/Roscosmos/ExoMars/CaSSIS. 


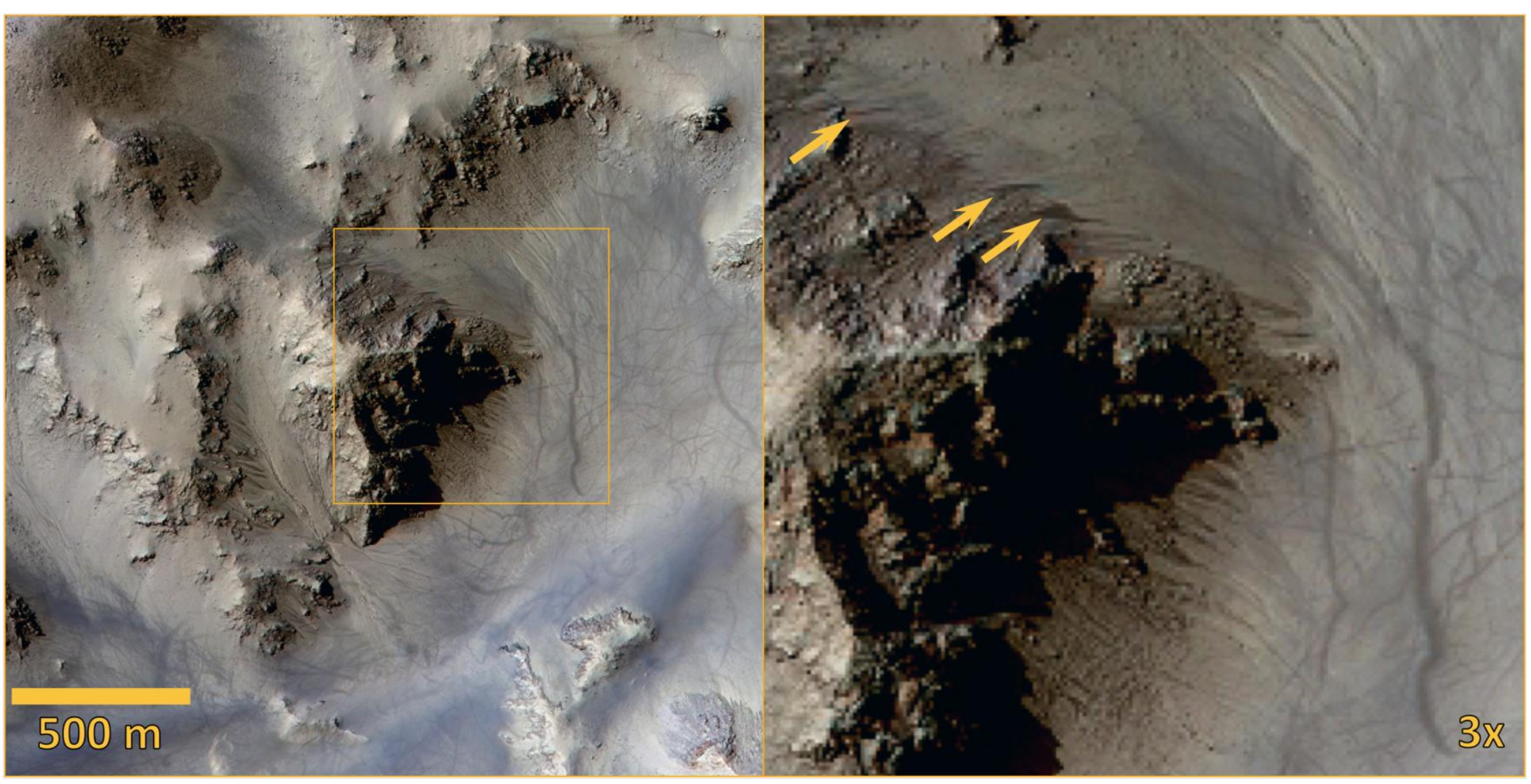

Figure 16 A fully-simulated CaSSIS infrared colour image (IRB1) of a portion of the central uplift of Horowitz crater $\left(140.82^{\circ} \mathrm{E},-32.05^{\circ} \mathrm{N}\right)$ known to host confirmed Recurring Slope Lineae (RSL). Three dark slope streaks (orange arrows) are HiRISE-confirmed RSLs observed in 2007 during southern summer $\left(L_{s}=334^{\circ}\right)(c . f$. ., Figure 1 in McEwen et al 2011). Several other RSLs are resolved by the fully-simulated CaSSIS image, but only where they occur as dense groupings of RSLs. This simulated image also shows some gully channels (upper right) and relatively dark dust devil streaks (right) in the area. Image credits: WesternU-CPSX/ASC-CSA/NASA, NASA/JPL/JHU-APL/MSSS/UofA and ESA/Roscosmos/ExoMars/CaSSIS. 


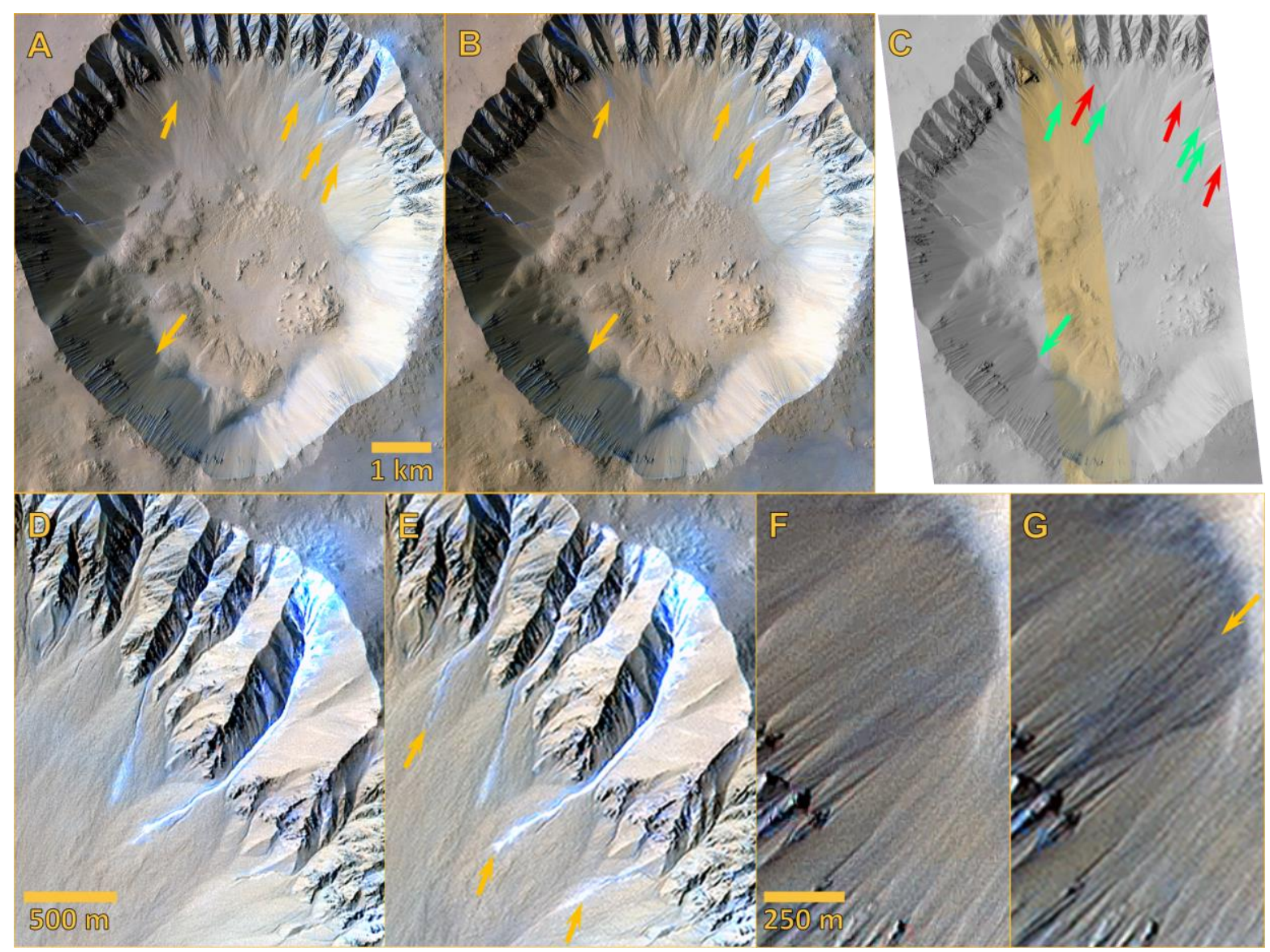

Figure 17 A comparison of two fully-simulated CaSSIS infrared colour (IRB1) images (A and B), and a cropped HiRISE MIRB image ESP_022718_1440 (C) of Gasa crater $\left(129.39^{\circ} \mathrm{E},-35.74^{\circ} \mathrm{N}\right)$. These images document changes that occurred between 2009 (A) and 2011 (B-C) and the anticipated ability of CaSSIS to detect these changes with its colour capability combined with its spatial coverage and resolution. Orange arrows show all locations where changes were observed in the simulated CaSSIS images. The green arrows in (C) indicate new or pre-existing light-toned gully deposits detected in both the HiRISE and simulated CaSSIS images. Red arrows indicate areas where a new or pre-existing light-toned gully deposit detected in the simulated CaSSIS images was difficult to detect in HiRISE, or was not covered with the narrow HiRISE colour strip. Changes from 20092011 in Gasa include both new gully deposits on the pole-facing slopes and a new mass wasting feature on the non-gullied southwestern crater wall. Please see the animated GIF of Gasa, based on the fully-simulated image pair in (A) and (B), provided with our supporting online supplementary materials. Image credits: WesternU-CPSX/ASC-CSA/NASA, NASA/JPL/JHU-APL/MSSS/UofA and ESA/Roscosmos/ExoMars/CaSSIS. 


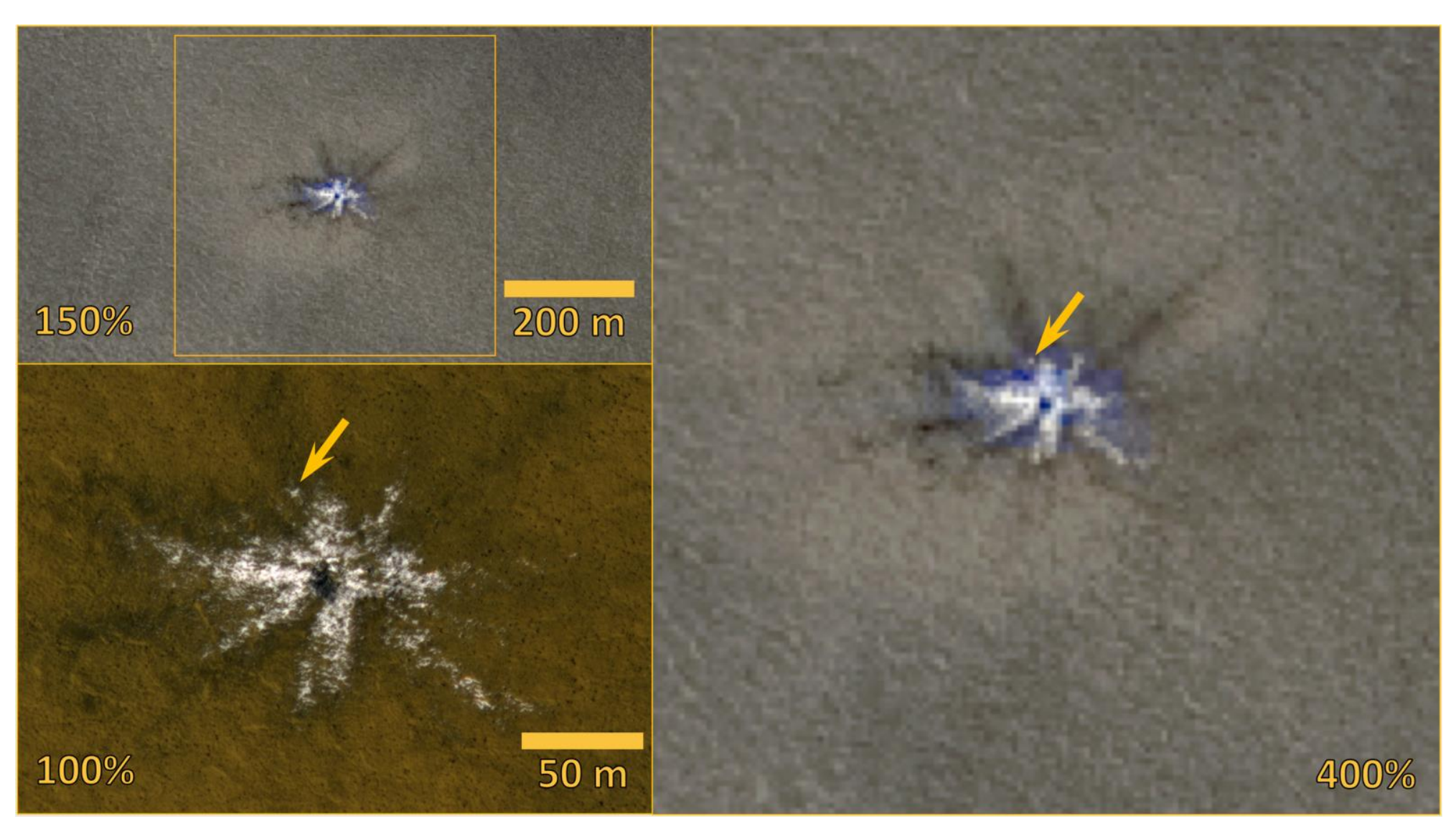

Figure 18 A comparison of a fully-simulated CaSSIS infrared colour image (IRB1) compared to a HiRISE IRB colour image of a recent ice-excavating crater that formed between March 2008 and March 2010 at $44.91^{\circ} \mathrm{E}, 63.92^{\circ} \mathrm{N}$. The coordinated HiRISE-CTX-CRISM image used to create the fully-simulated CaSSIS image (above left and right) was acquired on the $19^{\text {th }}$ of May 2010, at the onset of northern summer $\left(L_{s}=92.9^{\circ}\right)$. As can be seen here, the fully-simulated CaSSIS images (above left and right) compares remarkably well with the HiRISE IRB image ESP_017868_2440 (lower left). The dark-toned rayed ejecta, ice-distribution - including a small discrete patch of ejected ice (arrow) are all wellresolved. Image credits: WesternU-CPSX/ASC-CSA/NASA, NASA/JPL/JHU-APL/MSSS/UofA and ESA/Roscosmos/ExoMars/CaSSIS. 


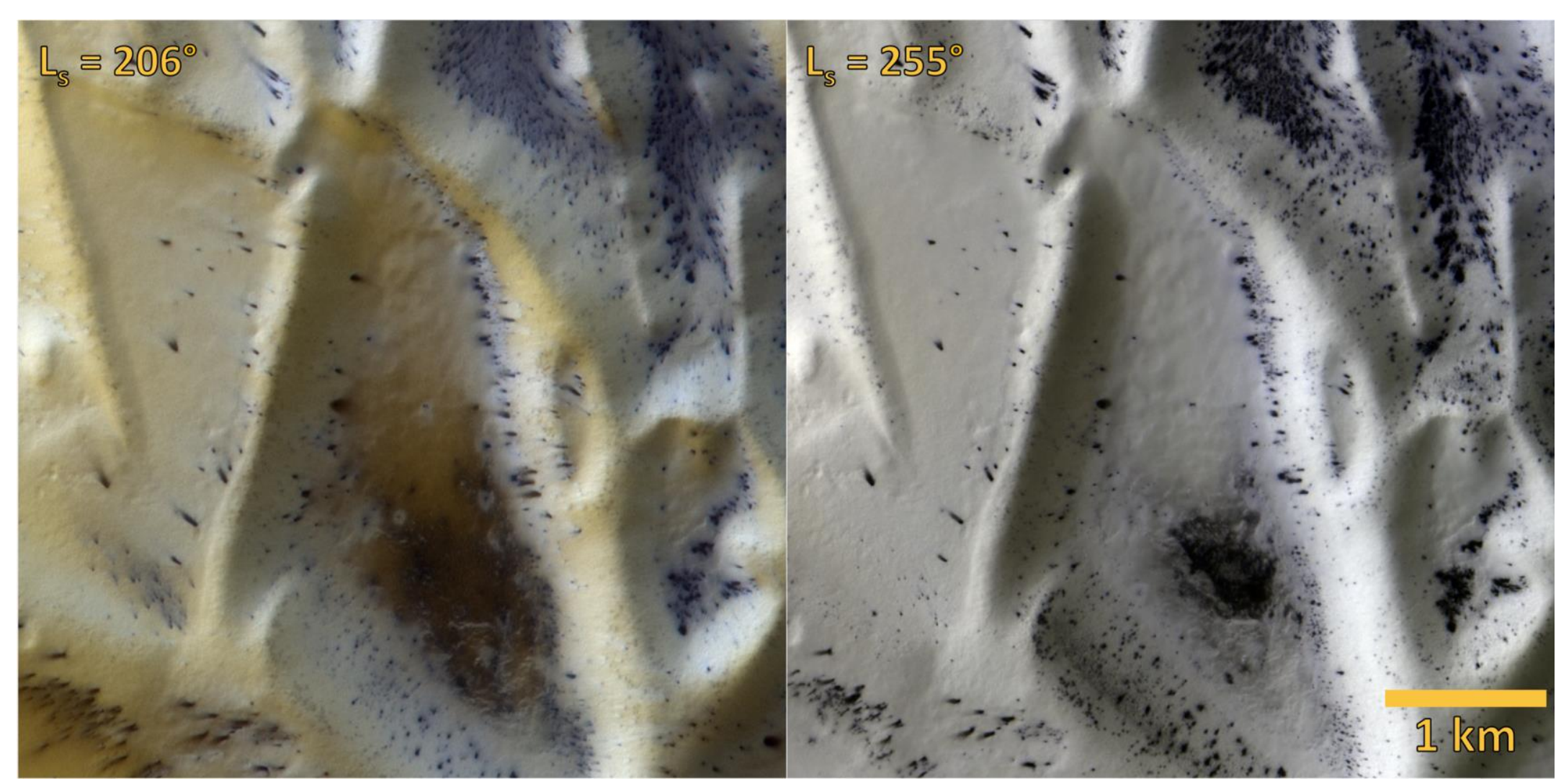

Figure 19 Fully-simulated CaSSIS infrared colour image (IRB1) change-detection pair covering the southern hemisphere site dubbed "Inca City," documenting $\mathrm{CO}_{2}$ frost sublimation during the transition from southern spring to summer $\left(295.81^{\circ} \mathrm{E}, 81.33^{\circ} \mathrm{S}\right)$. From one image to the next, the $L_{s}$ and incidence angles vary from $\sim 206^{\circ}$ to $255^{\circ}$ and $\sim 78^{\circ}$ to $62^{\circ}$, respectively. Please see the animated GIF provided of this image-set provided with our supporting online supplementary materials. Image credits: WesternUCPSX/ASC-CSA/NASA, NASA/JPL/JHU-APL/MSSS/UofA and ESA/Roscosmos/ExoMars/CaSSIS. 


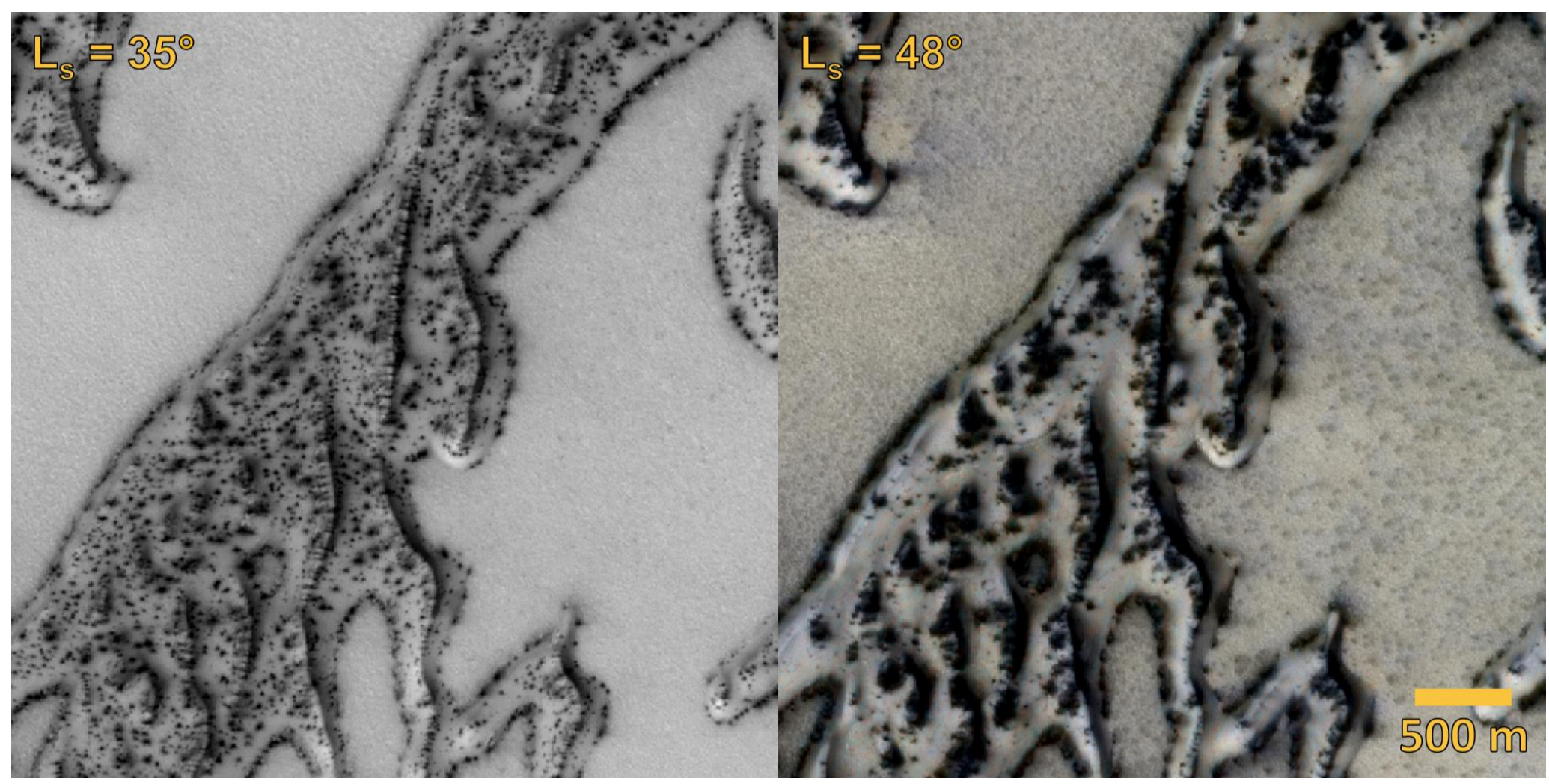

Figure 20 Fully-simulated CaSSIS infrared colour image (IRB1) change-detection pair covering the northern hemisphere site dubbed "Arrakis" dune field documenting $\mathrm{CO}_{2}$ ice defrosting during the onset of Northern spring $\left(122.58^{\circ} \mathrm{E}, 80.01^{\circ} \mathrm{N}\right)$. From one image to the next, the $L_{s}$ and incidence angles vary from $\sim 35^{\circ}$ to $48^{\circ}$ and $\sim 61^{\circ}$ to $59^{\circ}$, respectively. The simulated CaSSIS image on the right appears less sharp and blurred. This is due to high concentrations of water-ice aerosols, which impacted the clarity of the CRISM-CTX coordinated image set used to construct this simulation product (see Table 2). Despite this atmospheric issue, some of the "smearing" and merging of dark-toned spots observed from one image to the next is consistent with the modification of spots due to wind and aeolian activity (see HiRISE ESP_017100_2600 and ESP_017311_2600). Please see the animated GIF provided of this image-set provided with our supporting online supplementary materials. Image credits: WesternU-CPSX/ASC-CSA/NASA, NASA/JPL/JHU-APL/MSSS/UofA and ESA/Roscosmos/ExoMars/CaSSIS. 

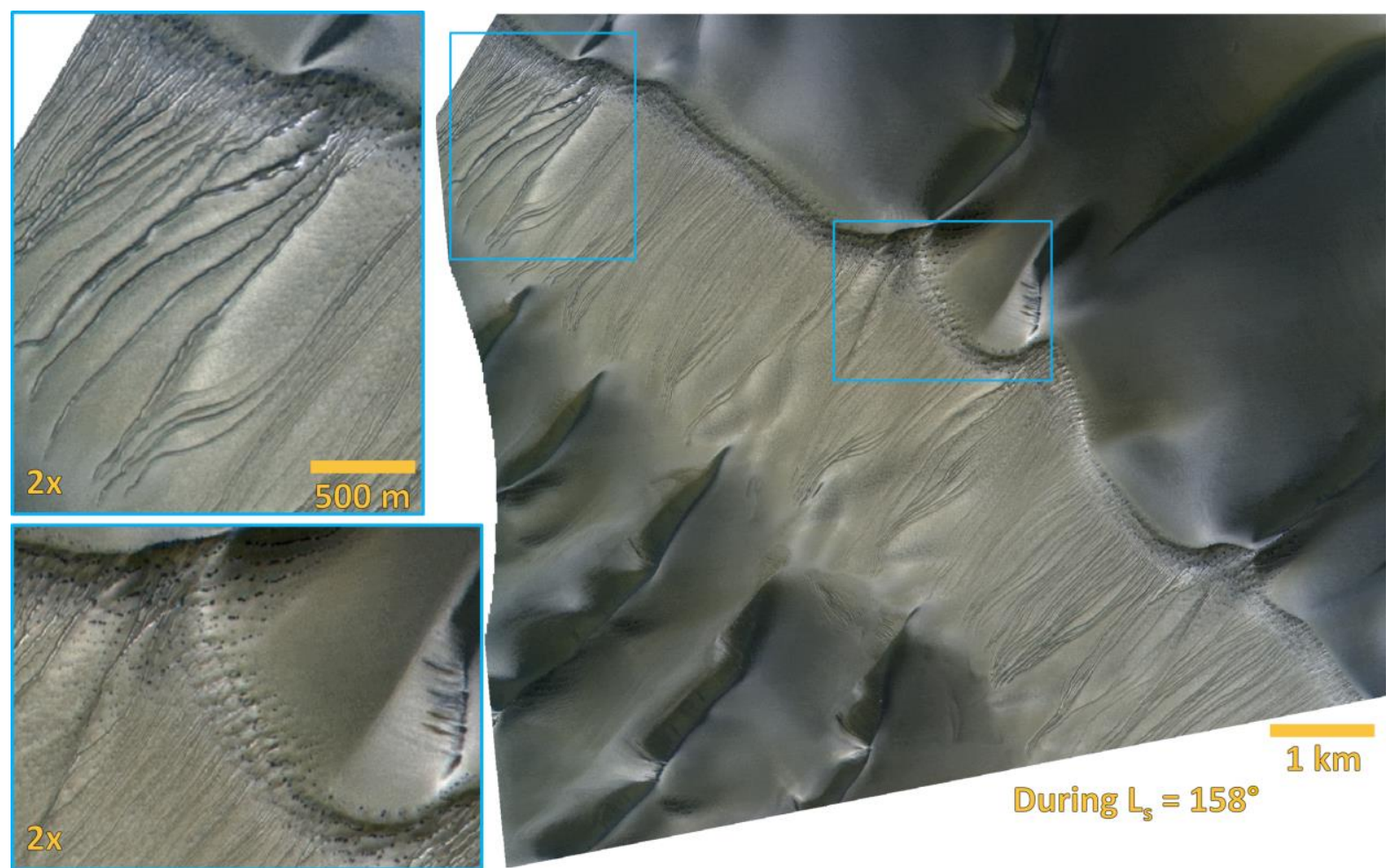

Figure 21 A fully-simulated CaSSIS infrared colour image (IRB1) covering the southern hemisphere site covering the NE portion of the Russell crater dune field $\left(12.94^{\circ} \mathrm{E}, 54.25^{\circ} \mathrm{S}\right)$, documenting seasonal activity associated with defrosting and sublimation of $\mathrm{CO}_{2}$ ice around the onset of southern spring $\left(\mathrm{L}_{s}=157.7^{\circ}\right)$ and an incidence of $\sim 80^{\circ}$. Image credits: WesternU-CPSX/ASC-CSA/NASA, NASA/JPL/JHU-APL/MSSS/UofA and ESA/Roscosmos/ExoMars/CaSSIS. 

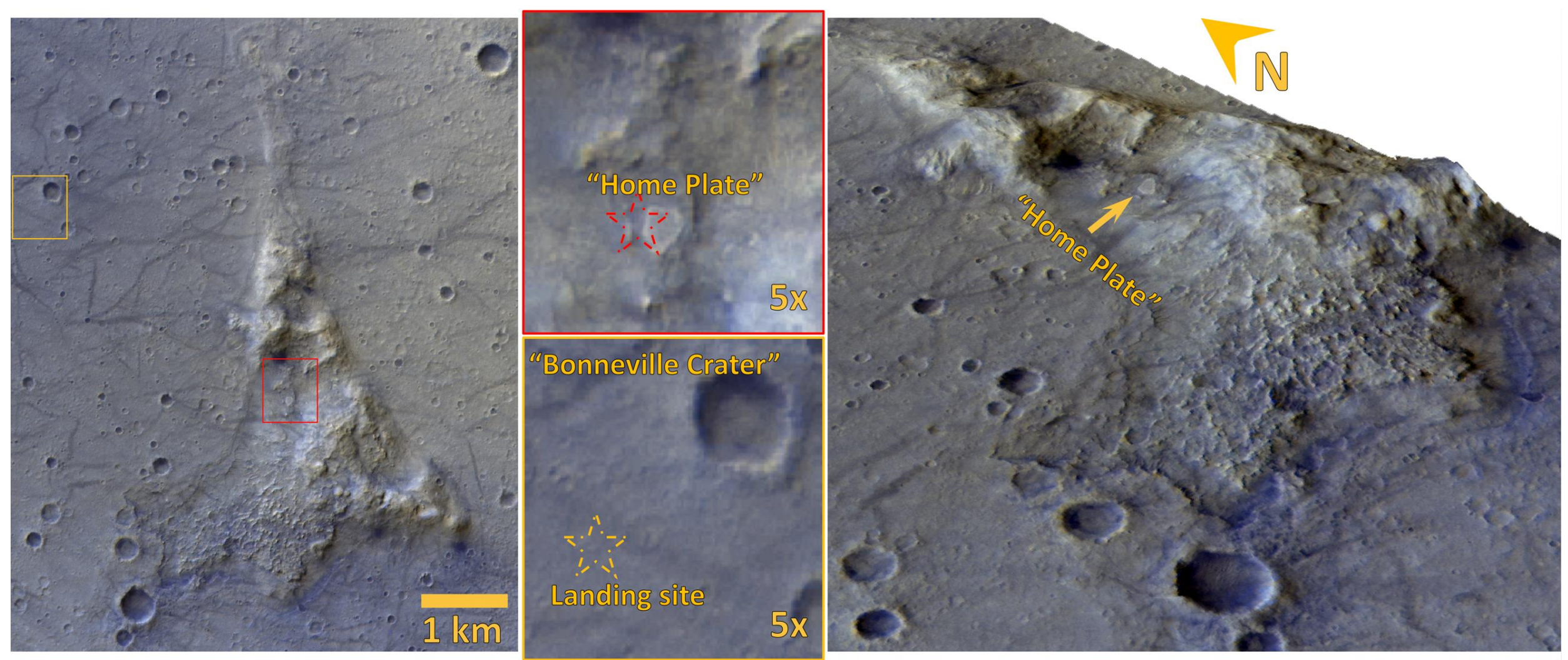

Figure 22 Portions of a fully-simulated CaSSIS infrared colour image (IRB1) and 3D perspective view of the Mars Exploration Rover (MER) Spirit landing site (orange star) and the Columbia Hills $\left(175.54^{\circ} \mathrm{E}, 14.62^{\circ} \mathrm{S}\right)$ in Gusev crater. The red star indicates the last known location of Spirit (see HiRISE ESP_016677_1650). The rover is clearly too small (1.6 x 2.3 meters) to be resolved by CaSSIS. Image credits: WesternU-CPSX/ASC-CSA/NASA, NASA/JPL/JHU-APL/MSSS/UofA and ESA/Roscosmos/ExoMars/CaSSIS. 

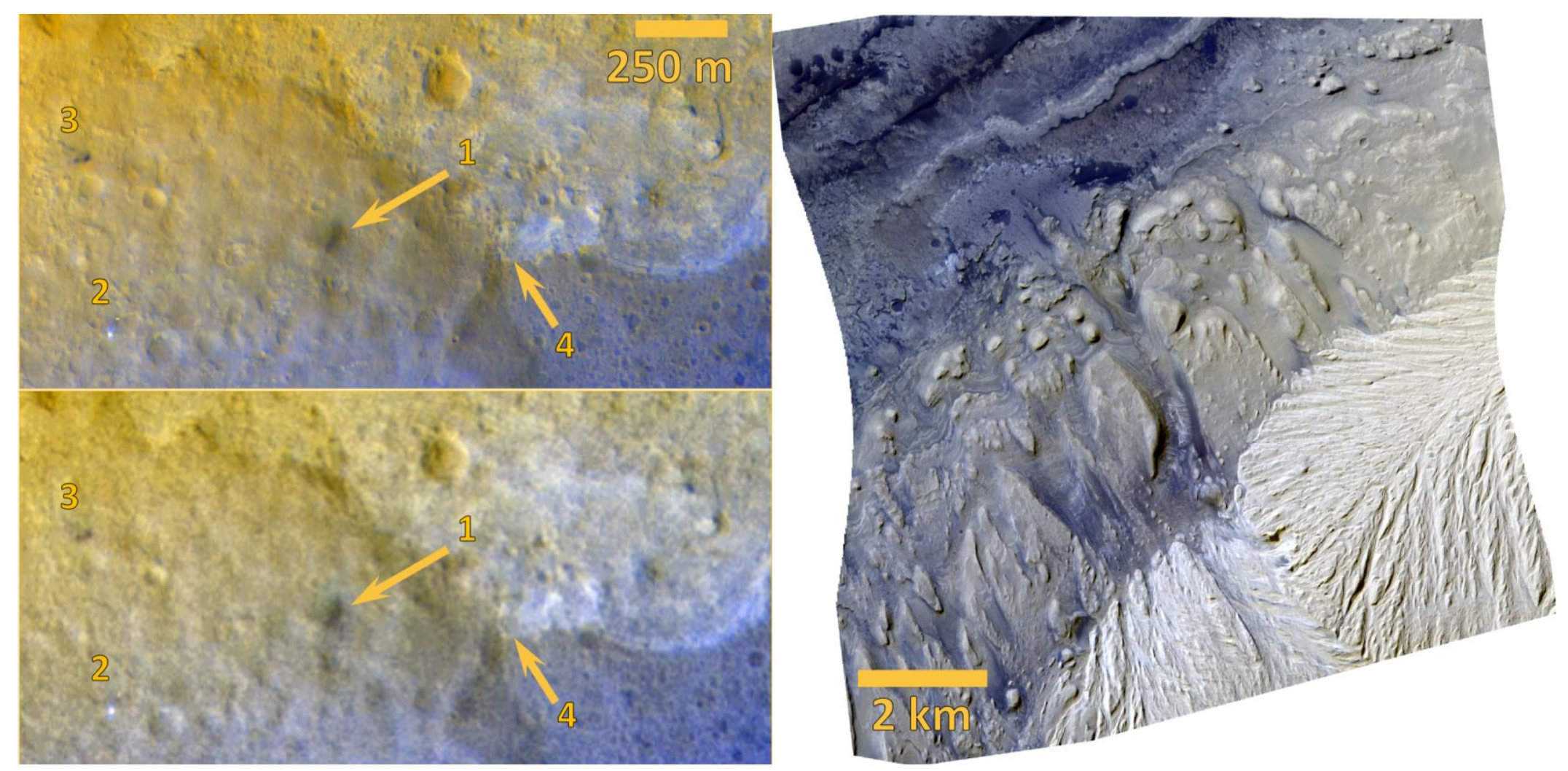

Figure 23 Fully-simulated CaSSIS infrared colour (IRB1) images of the Mars Science Lab (MSL) Curiosity landing site ("Bradbury Landing") and a portion of Aeolis Mons (a.k.a, "Mount Sharp"). Here we compare a HiRISE-based simulated CaSSIS image (above left) with one that is CTX-based (below left) centered at $137.45^{\circ} \mathrm{E}, 4.60^{\circ} \mathrm{S}$. In both simulations, landing site features, such as (1) "Bradbury Landing" landing site, (2) the parachute and backshell, and (3) the sky crane impact site, are all resolvable; however, and despite its relatively large size $(2.8 \times 3.0$ meters), nether the Curiosity rover, which was located at the Yellowknife Bay location (4) during the acquisition of the CRISM-CTXHiRISE coordinated images, nor the rover tracks leading there, can be resolved in our simulated images (see HiRISE ESP_030313_1755). The image on the right is a fullysimulated CaSSIS infrared colour image of a portion of "Mount Sharp" $\left(137.42^{\circ} \mathrm{E}, 4.79^{\circ} \mathrm{S}\right)$, which is currently being investigated by the MSL-Curiosity rover. Image credits: WesternU-CPSX/ASC-CSA/NASA, NASA/JPL/JHU-APL/MSSS/UofA and ESA/Roscosmos/ExoMars/CaSSIS. 


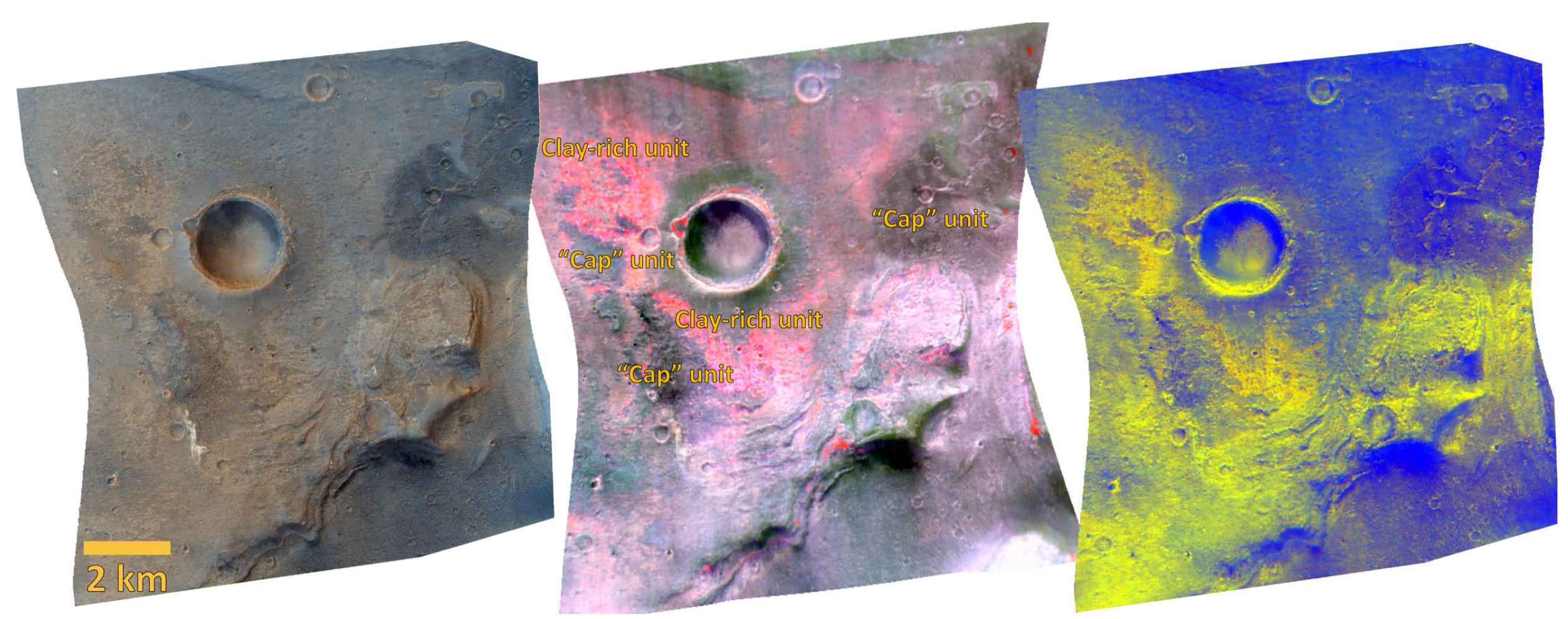

Figure 24 A comparison of fully-simulated CaSSIS colour images with a CRISM spectral parameter image composite covering the southeastern portion of the ExoMars 2020 landing ellipse in Oxia Planum ( $\left.336.1^{\circ} \mathrm{E}, 17.7^{\circ} \mathrm{N}\right)$. A fully-simulated CaSSIS synthetic "true" colour image (RGB) (left) and the CBRC1 image (right) highlight the differences between ferrous- (blue and purple) and ferric-bearing (orange and yellow) materials compared to a CRISM spectral parameter composite image (centre), which was constructed in a similar fashion to the one in Figure 14. The most intensely altered areas (strong magenta colour) shown by the CRISM composite is most distinctive in the simulated CaSSIS CBRC1 image (right) as an orange-coloured unit. Image credits: ASC-CSA/WesternU-CPSX, ESA/Roscosmos/ExoMars/CaSSIS and NASA/JPL/JHUAPL/MSSS/UofA. 

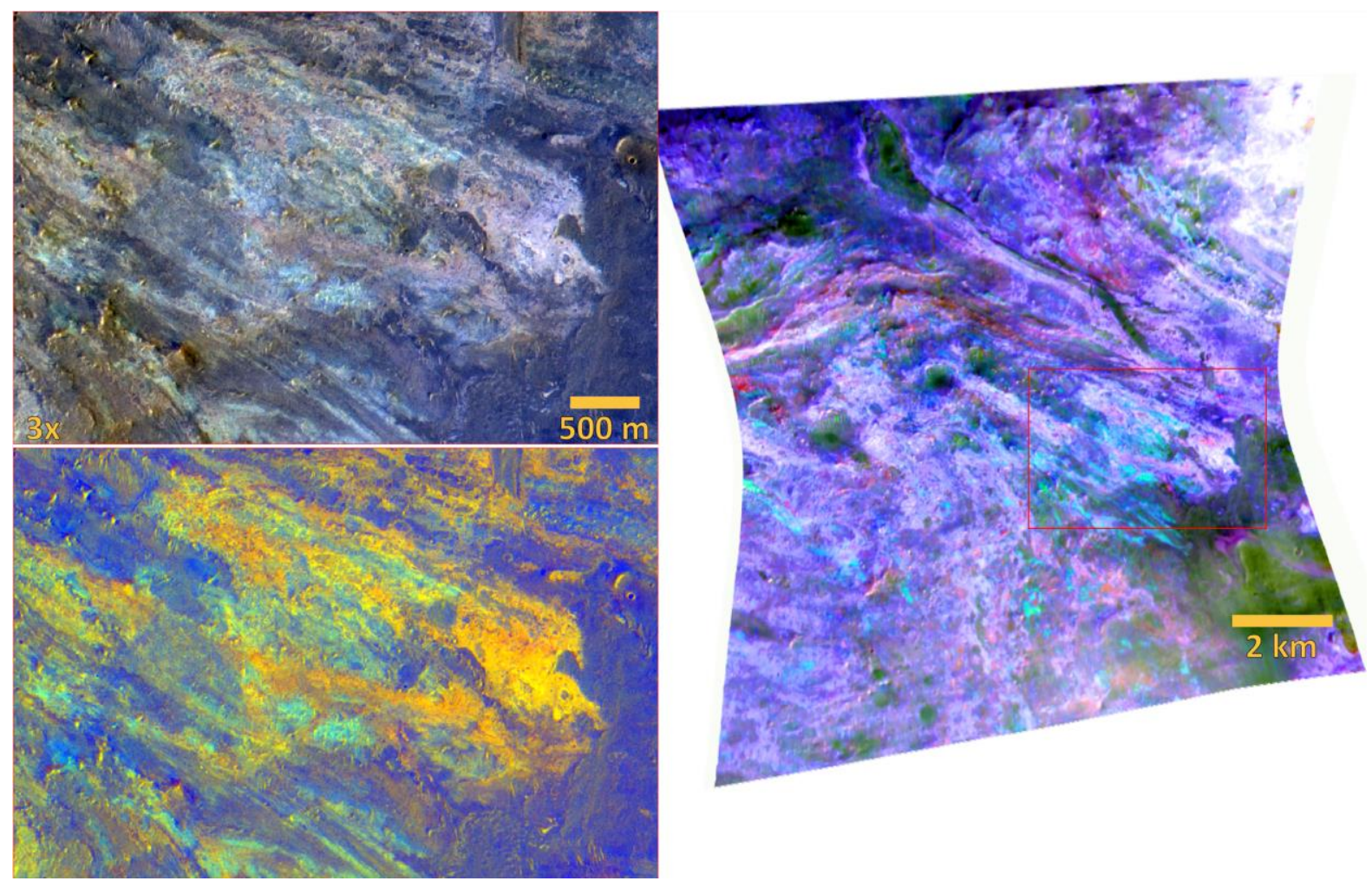

Figure 25 A comparison of a close-up fully-simulated CaSSIS colour images with a CRISM spectral parameter composite image covering a portion of the former Mars 2020 candidate landing site "Nili Fossae Trough" $\left(74.58^{\circ} \mathrm{E}, 21.05^{\circ} \mathrm{N}\right)$. The close-up infrared colour (IRB1) (upper left) and CBRC1 (lower left) images demonstrate the level of colour and morphologic detail of various landing sites provided by the anticipated CaSSIS dataset. The simulated images, and a context CRISM spectral parameter composite (constructed in a similar fashion to the one in Figure 14) provide details on the clast and matrix compositional diversity of the scoured and well-exposed Hargraves ejecta blanket, which occupies the majority of the proposed landing ellipse (>50\%). The sharp contacts between ferrous $\left(\mathrm{Fe}^{2+}\right)$ (blues) and ferric $\left(\mathrm{Fe}^{3+}\right)$ iron-bearing deposits (yellows and oranges) observed in the fully-simulated CaSSIS images (left) provide locations and potential sampling sites where redox chemical gradients occurr, and where past life may have possibly flourished. Image credits: ASC-CSA/WesternU-CPSX, ESA/Roscosmos/ExoMars/CaSSIS and NASA/JPL/JHU-APL/MSSS/UofA. 
Table 2 Datasets used to construct fully-simulated CaSSIS cubes

\begin{tabular}{|c|c|c|c|c|c|c|c|c|c|}
\hline \multirow[t]{2}{*}{ Feature or Location } & \multirow{2}{*}{$\begin{array}{l}\text { CRISM } \\
(18-20 \mathrm{~m} / \mathrm{pix})\end{array}$} & \multirow{2}{*}{$\begin{array}{l}\text { CTX } \\
(5-6 \mathrm{~m} / \mathrm{pix})\end{array}$} & \multirow{2}{*}{$\begin{array}{l}\text { HiRISE } \\
(25-50 \mathrm{~cm} / \text { pix })\end{array}$} & \multirow{2}{*}{$\begin{array}{l}{ }^{1} \text { Observatio } \\
\text { n Date }\end{array}$} & \multirow{2}{*}{$\begin{array}{c}{ }^{2} \text { Atmosphere } \\
\left(\tau_{\text {dust }} / \tau_{\text {ice }}\right)\end{array}$} & \multicolumn{4}{|c|}{${ }^{1}$ Observation Angles $\left({ }^{\circ}\right)$} \\
\hline & & & & & & Incid. & ${ }^{2}$ Emiss. & Phase & Ls \\
\hline $\begin{array}{l}\text { Russell Crater dunes } \\
\text { (Fig. 21) }\end{array}$ & FRT000039DF & P02_001981_1255_ & PSP_001981_1255 & $2006-12$ & $0.5-1.0 / 0-0.2$ & 80.66 & 8.79 & 73.8 & 157.7 \\
\hline $\begin{array}{l}\text { Nili Fossae (Fe Olivine and } \\
\text { Fe-Mg Carbonate) } \\
\text { (Figs. 3, 7, 8, 10, S2-S4) }\end{array}$ & FRT00003E12 & P03_002176_2024_ & PSP_002176_2025 & 2007-01 & $0.0-0.5 / 0-0.2$ & 53.94 & 6.07 & 60.01 & 165.8 \\
\hline "Inca City" ( ${ }^{3}$ Change & FRT00004F9B & P05_003092_0983_ & PSP_003092_0985 & 2007-03 & $0.5-1.0 / 0-0.2$ & 76.48 & 1.65 & 77.89 & 206.65 \\
\hline detection pair) (Fig. 19) & FRT0000629D & P08_004147_0985_ & PSP_004081_0985 & $2007-06$ & $1.0-1.5 / 0-0.2$ & 62.25 & 0.66 & 62.77 & 254.81 \\
\hline $\begin{array}{l}\text { Jezero Crater \& Mars } 2020 \\
\text { (Fig. S5 \& S10) }\end{array}$ & FRT00005C5E & P03_002387_1987_ & PSP_002387_1985 & 2007-05 & $0.5-1.0 / 0-0.2$ & 63.94 & 17.57 & 49.32 & 240.84 \\
\hline $\begin{array}{l}\text { Eberswalde Delta \& Mars } \\
2020 \text { (Fig. S11) }\end{array}$ & FRT000060DD & P01_001336_1560_ & PSP_001336_1560 & 2007-06 & $0.5-1.0 / 0-0.2$ & 44.52 & 1.85 & 42.67 & 250.81 \\
\hline $\begin{array}{l}\text { Alga Crater uplift } \\
\text { (Fig. 12) }\end{array}$ & FRT00006415 & P08_004158_1556_ & PSP_007573_1555 & $2007-06$ & $1.0-1.5 / 0-0.2$ & 42.79 & 6.35 & 36.45 & 258.62 \\
\hline $\begin{array}{l}\text { Nili Fossae Trough (LCP) \& } \\
\text { Mars } 2020 \text { (Figs. 9d \& S13) }\end{array}$ & FRT000064D9 & P07_003587_2005_ & PSP_003587_2015 & 2007-06 & $0.5-1.0 / 0-0.2$ & 62.64 & 3.26 & 65.18 & 261.69 \\
\hline $\begin{array}{l}\text { Ritchey Crater uplift } \\
\text { (Fig. 13) }\end{array}$ & FRT00007C34 & P11_005372_1514_ & PSP_005372_1515 & 2007-09 & $1.0-1.5 / 0-0.2$ & 33.6 & 7.93 & 41.16 & 316.31 \\
\hline $\begin{array}{l}\text { Oxia Planum (Capping unit) } \\
\text { \& ExoMars } 2020 \text { (Fig. 24) }\end{array}$ & FRT0000810D & P22_009735_1977_ & PSP_009735_1985 & $2007-10$ & $0.5-1.0 / 0-0.2$ & 45.21 & 1.34 & 46.22 & 326.13 \\
\hline Elorza Crater uplift (Fig. 12) & FRT00008236 & P12_005649_1696_ & PSP_005649_1710 & $2007-10$ & $1.0-1.5 / 0-0.2$ & 33.37 & 1.75 & 31.62 & 328.46 \\
\hline $\begin{array}{l}\text { Horowitz uplift RSL } \\
\text { (Fig. 16) }\end{array}$ & FRT00008573 & P12_005576_1473_ & PSP_005787_1475 & $2007-10$ & $0.5-1.0 / 0-0.2$ & 39.31 & 8.2 & 32.7 & 334.35 \\
\hline $\begin{array}{l}\text { Martin Crater uplift } \\
\text { (Fig. 12) }\end{array}$ & FRT00008649 & P12_005821_1585_ & PSP_005821_1585 & 2007-10 & $0.5-1.0 / 0-0.2$ & 35.08 & 2.12 & 33.09 & 335.78 \\
\hline
\end{tabular}




\begin{tabular}{|c|c|c|c|c|c|c|c|c|c|}
\hline $\begin{array}{l}\text { Mawrth Vallis (Craters) } \\
\text { ExoMars \& Mars } 2020 \\
\text { (Fig. S12) }\end{array}$ & FRT000094F6 & P15_006821_2045_ & PSP_006821_2045 & $2008-01$ & $0.0-0.5 / 0-0.2$ & 39.42 & 7.34 & 46.58 & 15.26 \\
\hline $\begin{array}{l}\text { Oxia Planum (“Delta”) \& } \\
\text { ExoMars } 2020 \text { (Fig. S9) }\end{array}$ & FRT00009A16 & P15_007019_1978_ & PSP_007019_1980 & $2008-01$ & $0.5-1.0 / 0-0.2$ & 38.71 & 0.49 & 39.17 & 22.54 \\
\hline $\begin{array}{l}\text { Nili Fossae (Talc) } \\
\text { (Figs. 9s \& 14) }\end{array}$ & FRT00009D44 & P16_007200_2002_ & PSP_007200_2005 & $2008-02$ & $0.0-0.5 / 0-0.2$ & 39.18 & 1.44 & 40.62 & 29.08 \\
\hline $\begin{array}{l}\text { Mawrth Vallis ExoMars \& } \\
\text { Mars } 2020 \text { (Fig. S12) }\end{array}$ & FRT0000A600 & P17_007612_2043_ & PSP_007612_2045 & $2008-03$ & $0.0-0.5 / 0-0.2$ & 38.84 & 20.85 & 59.63 & 43.62 \\
\hline $\begin{array}{l}\text { Nili Fossae (Kaolinite \& } \\
\text { Serpentine) } \\
\text { (Fig. } 9 \mathrm{~m} \text { \& see Fig. } 28 \text { in } \\
\text { Thomas et al this issue) }\end{array}$ & FRTO000ABCB & P19_008347_2019_ & No HiRISE coverage & $2008-05$ & $0.0-0.5 />0.2$ & 43.44 & 0.15 & 43.32 & 68.83 \\
\hline $\begin{array}{l}\text { Nili Fossae Trough \& Mars } \\
2020 \text { (Fig. 25) }\end{array}$ & FRT0000B012 & P20_008782_1999_ & PSP_008782_2015 & $2008-06$ & $0.0-0.5 / 0-0.2$ & 45.17 & 5.79 & 39.8 & 83.65 \\
\hline Toro Crater Uplift & FRT0000B1B5 & P12_005842_1964_ & PSP_005842_1970 & $2008-06$ & $0.0-0.5 />0.2$ & 45.91 & 4.96 & 50.39 & 85.91 \\
\hline $\begin{array}{l}\text { Aeolis Mons ("Mount } \\
\text { Sharp") \& MSL-Curiosity } \\
\text { (Fig. 23) }\end{array}$ & FRT0000B6F1 & P21_009149_1752_ & PSP_009149_1750 & 2008-07 & $0.5-1.0 / 0-0.2$ & 58.86 & 14.34 & 48.46 & 96.28 \\
\hline Chlorides (Fig. 11) & FRT00010A4E & B05_011691_1409_ & PSP_003160_1410 & 2009-01 & $0.5-1.0 / 0-0.2$ & 62.5 & 4.93 & 57.96 & 196.58 \\
\hline Negril crater uplift & FRT000110B7 & B06_011960_2004_ & ESP_025766_2005 & 2009-02 & $0.5-1.0 / 0-0.2$ & 64.11 & 6.38 & 58.03 & 209.14 \\
\hline $\begin{array}{l}\text { Unnamed crater uplift } \\
\text { (Fig. 12) }\end{array}$ & FRT00012B01 & B09_013134_1611_ & ESP_013134_1610 & $2009-05$ & $0.5-1.0 / 0-0.2$ & 43.08 & 7.1 & 36.19 & 266.54 \\
\hline $\begin{array}{l}\text { Verlaine Crater uplift } \\
\text { (Fig. 12) }\end{array}$ & FRT00012CD9 & B09_013213_1707_ & ESP_013213_1705 & 2009-05 & $0.5-1.0 / 0-0.2$ & 44.82 & 1.53 & 43.34 & 270.42 \\
\hline Gasa Crater gullies & FRT00013F9F & B11_014081_1440_- & ESP_014081_1440 & 2009-07 & 0.0-0.5 / 0-0.2 & 36.65 & 0.82 & 37.35 & $\begin{array}{l}311.52 \\
30331\end{array}$ \\
\hline $\begin{array}{l}\text { ( }{ }^{3} \text { Change detection pair) } \\
\text { (Fig. 17) }\end{array}$ & FRIOUOIESAL & G12_022/18_1439 & ESP_022/18_1440 & $2011-06$ & $0.5-1.0 / 0-0.2$ & 34.16 & 2.15 & 32.29 & 303.31 \\
\hline
\end{tabular}




\begin{tabular}{|c|c|c|c|c|c|c|c|c|c|}
\hline $\begin{array}{l}\text { Columbia Hills, MER-Spirit } \\
\text { \& Mars } 2020 \text { (Figs. } 22 \text { \& } \\
\text { S8) }\end{array}$ & FRT000168F3 & B18_016677_1653_ & ESP_016677_1650 & $2010-2$ & $0.0-0.5 / 0-0.2$ & 57.04 & 7.36 & 51.85 & 52.23 \\
\hline $\begin{array}{l}\text { "Arrakis" dune field } \\
\left({ }^{3} \text { Change detection pair) }\right. \\
\text { (Fig. 20) }\end{array}$ & $\begin{array}{l}\text { FRT00016B45 } \\
\text { FRT000175A2 }\end{array}$ & $\begin{array}{l}\text { B18_016744_2601_- } \\
\text { B19_017100_2601_ }\end{array}$ & $\begin{array}{l}\text { ESP_016744_2600 } \\
\text { ESP_017100_2600 }\end{array}$ & $\begin{array}{l}2010-2 \\
2010-3\end{array}$ & $\begin{array}{l}0.0-0.5 />0.2 \\
\text { Not Available }\end{array}$ & $\begin{array}{l}61.28 \\
58.67\end{array}$ & $\begin{array}{l}0.87 \\
2.07\end{array}$ & $\begin{array}{l}61.93 \\
60.34\end{array}$ & $\begin{array}{l}54.54 \\
66.69\end{array}$ \\
\hline $\begin{array}{l}\text { Recent ice-excavating } \\
\text { impact (Fig. 18) }\end{array}$ & FRT00018E24 & B21_017868_2451_ & ESP_017868_2440 & 2010-05 & Not Available & 47.73 & 6.98 & 54.13 & 92.92 \\
\hline $\begin{array}{l}\text { Unnamed crater uplift } \\
\text { (Fig. 12) }\end{array}$ & FRT0002367C & D17_033757_1513_ & ESP_028509_1510 & $2012-03$ & Not Available & 71.1 & 2.42 & 69.63 & 78.18 \\
\hline $\begin{array}{l}\text { Bradbury Landing } \\
\text { Curiosity-MSL (Fig. 23) }\end{array}$ & FRS00028346 & D08_030313_1751_ & ESP_030313_1755 & 2013-01 & Not Available & 50.34 & 4.45 & 46.14 & 244.14 \\
\hline
\end{tabular}

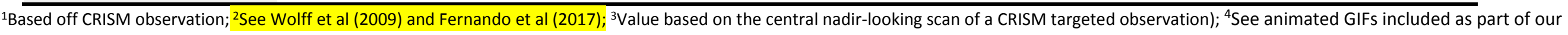
online supplemental materials; *Red text denotes non-coordinated, atmospheric dust and/or ice aerosol contamination, and high emission $\left(>9^{\circ}\right)$ observations 
Table 3 CaSSIS Band Ratios or Spectral Parameters for Distinguishing Common Surface Materials and Atmospheric Phenomena

\section{Product \\ 2PAN/1BLU \\ (Figs. 7 \& S6)}

\section{PAN/4NIR}

(Figs. $7 \&$ S6)

\section{RED/2PAN}

(Fig. 7)

Mean[1BLU-4NIR]/(4NIR/1BLU)

(Figs. 6 \& S6)

\section{Colour Band Ratio Composites}

CBRC1 (Figs. 7, 9, 11, 14, 24, 25, S9S13)

CBRC2 (Fig. S6)
${ }^{1}$ Sensitivity

Ferric iron $\left(\mathrm{Fe}^{3+}\right)$

Description

Absorption short of $\sim 550 \mathrm{~nm}$ due to

intervalence charge transfer of $\mathrm{Fe}^{3+}$

Ferrous iron Broad absorption centered around 1000-

$\left(\mathrm{Fe}^{2+}\right)$

$1100 \mathrm{~nm}$ due to crystal field transitions from the presence of $\mathrm{Fe}^{2+}$

Ferric iron $\left(\mathrm{Fe}^{3+}\right) \quad$ Small broad absorption (e.g., $~ 860 \mathrm{~nm}$ absorption for hematite) and slope around 600-700 $\mathrm{nm}$ due to crystal field transitions from the presence of $\mathrm{Fe}^{3+}$

Ices, Frosts, some Ices and some atmospheric phenomena (e.g. atmospheric phenomena

haze, fog, clouds, dust devils, avalanche

clouds, etc.), compared to other surface

materials, would have the highest reflectance

values in all 4 CaSSIS bands (e.g., very high

mean reflectance) and are most distinctive

on Mars with respect to blue wavelengths, a

NIR- or red-to-blue ratio and albedo (see text

for more details)

\section{Distinguishes}

Ferric, and Fe-

$3 / 2,2 / 1,2 / 4$ in R-G-B

N/A

ave for description of individual

ratios)

\section{Distinguishes $\quad 3 / 2,2 / 1$, Mean[1-4]/(4/1) in R-G-B}

Ice/atmospheric (see above for description of individual

phenomena from ratios)

other surface
Min./phase examples

${ }^{2}$ Alternates

Hematite, goethite, akaganeite

$$
\text { nontronite }
$$

$3 / 1,4 / 1$

Low-Ca pyroxenes, olivines, high- $\quad 3 / 4,2 / 3$

Ca proxenes

$$
\text { Nontronite, Hematite None }
$$

$\mathrm{H}_{2} \mathrm{O}, \mathrm{CO}_{2}$, Fog, Haze, Clouds

Mean[1-3]/(3/1),

Mean[1,2]/(2/1), or

Band 1 (blue-green)

image

${ }^{1}$ Higher values provided by these band ratios or spectral parameters signifies the likelihood of the presence of the materials listed under this heading as a surface component ${ }^{2}$ Band numbers are as follows: 1 - BLU (499.9 nm), 2 - PAN (675.0 nm), 3-RED (836.2 nm) and NIR (936.7 nm) 